\title{
Optimal time for initiating antiretroviral therapy (ART) in HIV-infected, treatment-naive children aged 2 to 5 years old (Review)
}

\author{
Siegfried N, Davies MA, Penazzato M, Muhe LM, Egger M
}

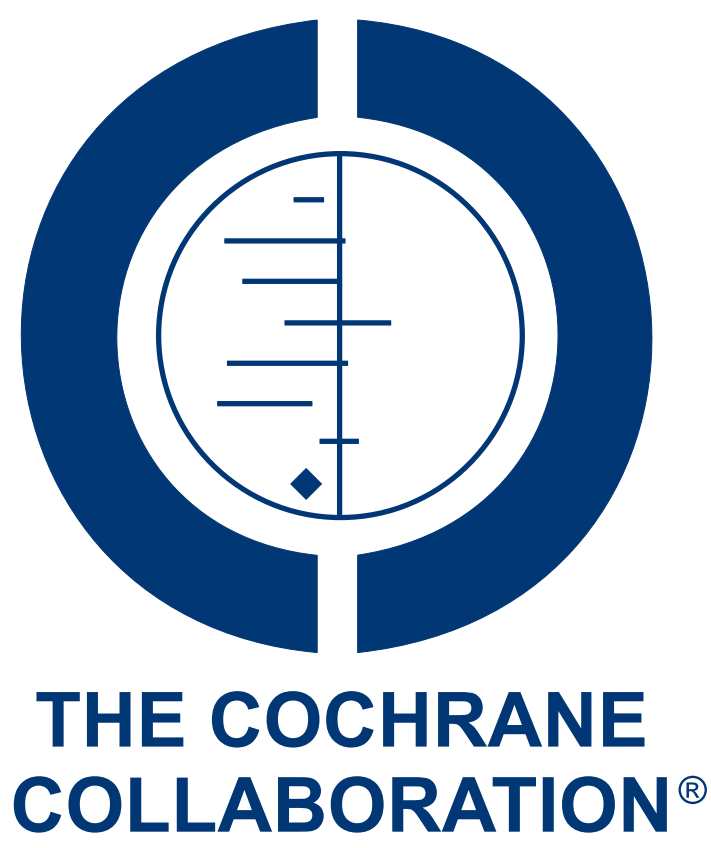

This is a reprint of a Cochrane review, prepared and maintained by The Cochrane Collaboration and published in The Cochrane Library 2013, Issue 10

http://www.thecochranelibrary.com

\section{WILEY}

Optimal time for initiating antiretroviral therapy (ART) in HIV-infected, treatment-naive children aged 2 to 5 years old (Review) Copyright $\odot 2013$ The Cochrane Collaboration. Published by John Wiley \& Sons, Ltd. 
TABLE OF CONTENTS

HEADER . . . . . . . . . . . . . . . . . . . . . . . . . . . . . . . . . . . . . . . . . . . . . . . . . . . . .

ABSTRACT . . . . . . . . . . . . . . . . . . . . . . . . . . . . . . . . . . . . . . . . . . . . . . . . . . .

PLAIN LANGUAGE SUMMARY . . . . . . . . . . . . . . . . . . . . . . . . . . . . . . . . . . . . . . . . . . . . .

SUMMARY OF FINDINGS FOR THE MAIN COMPARISON ．．．．．．．． . . . . . . . . . . . . . . . 3

BACKGROUND . . . . . . . . . . . . . . . . . . . . . . . . . . . . . . . . . . . . . . 6

OBJECTIVES . . . . . . . . . . . . . . . . . . . . . . . . . . . . . . . . . . . . . . . . . . . .

METHODS . . . . . . . . . . . . . . . . . . . . . . . . . . . . . . . . . . . . . . . .

RESULTS . . . . . . . . . . . . . . . . . . . . . . . . . . . . . . . . . . . . . . . . . . . .

Figure 1. . . . . . . . . . . . . . . . . . . . . . . . . . . . . . . . . . . . . . . .

Figure 2. . . . . . . . . . . . . . . . . . . . . . . . . . . . . . . . . . . . . . . .

Figure 3. . . . . . . . . . . . . . . . . . . . . . . . . . . . . . . . . . . . 15

ADDITIONAL SUMMARY OF FINDINGS . . . . . . . . . . . . . . . . . . . . . . . . . . . . . . .

DISCUSSION . . . . . . . . . . . . . . . . . . . . . . . . . . . . . . . . . . . . . . 25

AUTHORS' CONCLUSIONS . . . . . . . . . . . . . . . . . . . . . . . . . . . . . . . . . . . . .

ACKNOWLEDGEMENTS . . . . . . . . . . . . . . . . . . . . . . . . . . . . . . . . . . . . . . .

REFERENCES . . . . . . . . . . . . . . . . . . . . . . . . . . . . . . . . . . . . . . . 26

CHARACTERISTICS OF STUDIES . . . . . . . . . . . . . . . . . . . . . . . . . . . . . . . . . . . . . . . . .

DATA AND ANALYSES . . . . . . . . . . . . . . . . . . . . . . . . . . . . . . . . . . . . . . . . . . . 40

Analysis 1.1. Comparison 1 IMMEDIATE versus DEFERRED initiation of ART all ages (RCT), Outcome 1 Death. 42

Analysis 1.2. Comparison 1 IMMEDIATE versus DEFERRED initiation of ART all ages (RCT), Outcome 2 CDC

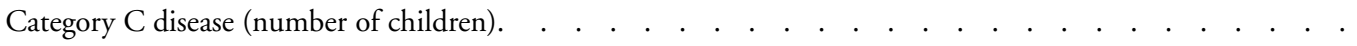

Analysis 1.3. Comparison 1 IMMEDIATE versus DEFERRED initiation of ART all ages (RCT), Outcome 3 CDC Category B disease (numbers of children) Relative Risk. . . . . . . . . . . . . . . . . . . . . . . . . . . . .

Analysis 1.4. Comparison 1 IMMEDIATE versus DEFERRED initiation of ART all ages (RCT), Outcome 4 CDC Category B disease (numbers of children) Peto Odds Ratio. . . . . . . . . . . . . . . . . . . . 45

Analysis 1.5. Comparison 1 IMMEDIATE versus DEFERRED initiation of ART all ages (RCT), Outcome 5 Pulmonary TB (clinically diagnosed). . . . . . . . . . . . . . . . . . . . . . . . . . . . . . . . . . . . . . . . . .

Analysis 1.7. Comparison 1 IMMEDIATE versus DEFERRED initiation of ART all ages (RCT), Outcome 7 Proportion of children on ART with HIV-RNA < 50 copies/ml.

Analysis 1.9. Comparison 1 IMMEDIATE versus DEFERRED initiation of ART all ages (RCT), Outcome 9 Mean CD4\% at week 144 .

Analysis 1.10. Comparison 1 IMMEDIATE versus DEFERRED initiation of ART all ages (RCT), Outcome 10 Proportion of children with $\mathrm{CD} 4 \%<15 \%$ at study end. . . . . . . . . . . . . . . . . . . . . . . . . . . . . . . . . .

Analysis 1.11. Comparison 1 IMMEDIATE versus DEFERRED initiation of ART all ages (RCT), Outcome 11 Mean weight gain per year in $\mathrm{kg}$.

Analysis 1.13. Comparison 1 IMMEDIATE versus DEFERRED initiation of ART all ages (RCT), Outcome 13 Mean height gain per year in $\mathrm{cm}$.

Analysis 1.15. Comparison 1 IMMEDIATE versus DEFERRED initiation of ART all ages (RCT), Outcome 15 Mean standardized score on Beery VMI at 144 weeks.

Analysis 1.16. Comparison 1 IMMEDIATE versus DEFERRED initiation of ART all ages (RCT), Outcome 16 Proportion of children with adverse events. . . . . . . . . . . . . . . . . . . . . . . . . . . . . . . . . . . . 50

Analysis 1.17. Comparison 1 IMMEDIATE versus DEFERRED initiation of ART all ages (RCT), Outcome 17 Proportion of children with ART-related adverse events.

Analysis 2.1. Comparison 2 SUBGROUP ANALYSIS: IMMEDIATE versus DEFERRED initiation of ART 24 to 59 months (RCT), Outcome 1 Death.

Analysis 2.2. Comparison 2 SUBGROUP ANALYSIS: IMMEDIATE versus DEFERRED initiation of ART 24 to 59 months (RCT), Outcome 2 CDC Category C disease (number of children). . . . . . . . . . . . . . . . . . .

Analysis 2.3. Comparison 2 SUBGROUP ANALYSIS: IMMEDIATE versus DEFERRED initiation of ART 24 to 59 months (RCT), Outcome 3 CDC Category B disease (numbers of children) Relative Risk.

Analysis 2.4. Comparison 2 SUBGROUP ANALYSIS: IMMEDIATE versus DEFERRED initiation of ART 24 to 59 months (RCT), Outcome 4 CDC Category B disease (numbers of children) Peto Odds Ratio.

Optimal time for initiating antiretroviral therapy (ART) in HIV-infected, treatment-naive children aged 2 to 5 years old (Review)

Copyright (๑) 2013 The Cochrane Collaboration. Published by John Wiley \& Sons, Ltd. 
Analysis 2.5. Comparison 2 SUBGROUP ANALYSIS: IMMEDIATE versus DEFERRED initiation of ART 24 to 59 months (RCT), Outcome 5 Pulmonary TB (clinically diagnosed). . . . . . . . . . . . . . . . . . . . . .

Analysis 2.6. Comparison 2 SUBGROUP ANALYSIS: IMMEDIATE versus DEFERRED initiation of ART 24 to 59 months (RCT), Outcome 6 Proportion of children on ART with HIV-RNA < 50 copies $/ \mathrm{ml} . \quad$. . . . . . .

Analysis 2.7. Comparison 2 SUBGROUP ANALYSIS: IMMEDIATE versus DEFERRED initiation of ART 24 to 59 months (RCT), Outcome 7 Mean CD4\% at week 144. . . . . . . . . . . . . . . . . . . . . . . . . . . .

Analysis 2.8. Comparison 2 SUBGROUP ANALYSIS: IMMEDIATE versus DEFERRED initiation of ART 24 to 59 months (RCT), Outcome 8 Proportion of children with CD $4 \%<15 \%$ at study end. . . . . . . . . . . . .

Analysis 2.9. Comparison 2 SUBGROUP ANALYSIS: IMMEDIATE versus DEFERRED initiation of ART 24 to 59 months (RCT), Outcome 9 Mean weight gain per year in kg. $\quad . \quad$. . . . . . . . . . . . . . . . . . . . . .

Analysis 2.10. Comparison 2 SUBGROUP ANALYSIS: IMMEDIATE versus DEFERRED initiation of ART 24 to 59 months (RCT), Outcome 10 Mean height gain per year in cm. . . . . . . . . . . . . . . . . . . . . . .

Analysis 2.11. Comparison 2 SUBGROUP ANALYSIS: IMMEDIATE versus DEFERRED initiation of ART 24 to 59 months (RCT), Outcome 11 Mean standardized score on Beery VMI at 144 weeks. . . . . . . . . . . . . .

Analysis 2.12. Comparison 2 SUBGROUP ANALYSIS: IMMEDIATE versus DEFERRED initiation of ART 24 to 59 months (RCT), Outcome 12 Proportion of children with ART-related Grade 3 or 4 adverse events. . . . . .

Analysis 3.1. Comparison 3 ADJUSTED/WEIGHTED EARLY vs DEFERRED initiation of ART in children with TB and HIV, Outcome 1 Death.

Analysis 3.2. Comparison 3 ADJUSTED/WEIGHTED EARLY vs DEFERRED initiation of ART in children with TB and HIV, Outcome 2 Virologic suppression. . . . . . . . . . . . . . . . . . . . . . . . 59

APPENDICES . . . . . . . . . . . . . . . . . . . . . . . . . . . . . . . . . . . . . . . . . . . .

CONTRIBUTIONS OF AUTHORS . . . . . . . . . . . . . . . . . . . . . . . . . . . . . . . . . . . . 66

DECLARATIONS OF INTEREST . . . . . . . . . . . . . . . . . . . . . . . . . . . . . . . . . . . . . . . . . 6

SOURCES OF SUPPORT . . . . . . . . . . . . . . . . . . . . . . . . . . . . . . . . . . . . . . . . . . . . . . . . .

Optimal time for initiating antiretroviral therapy (ART) in HIV-infected, treatment-naive children aged 2 to 5 years old (Review) 


\title{
[Intervention Review]
}

\section{Optimal time for initiating antiretroviral therapy (ART) in HIV-infected, treatment-naive children aged 2 to 5 years old}

\author{
Nandi Siegfried ${ }^{1,2}$, Mary-Ann Davies ${ }^{3}$, Martina Penazzato ${ }^{4,5}$, Lulu M Muhe ${ }^{4}$, Matthias Egger ${ }^{6}$ \\ ${ }^{1}$ Department of Psychiatry and Mental Health, University of Cape Town, Cape Town, South Africa. ${ }^{2}$ Department of Epidemiology and \\ Biostatistics, University of California, San Francisco, San Francisco, California, USA. ${ }^{3}$ Centre for Infectious Disease Epidemiology and \\ Research (CIDER), School of Public Health and Family Medicine, Cape Town, South Africa. ${ }^{4}$ World Health Organization, Geneva, \\ Switzerland. ${ }^{5}$ MRC Clinical Trials Unit, London, UK. ${ }^{6}$ Institute of Social Medicine, Institute of Social and Preventive Medicine, Bern, \\ Switzerland
}

Contact address: Nandi Siegfried, Department of Epidemiology and Biostatistics, University of California, San Francisco, San Francisco, California, USA. nandi.siegfried@gmail.com.

Editorial group: Cochrane HIV/AIDS Group.

Publication status and date: New, published in Issue 10, 2013.

Review content assessed as up-to-date: 24 May 2013.

Citation: Siegfried N, Davies MA, Penazzato M, Muhe LM, Egger M. Optimal time for initiating antiretroviral therapy (ART) in HIVinfected, treatment-naive children aged 2 to 5 years old. Cochrane Database of Systematic Reviews 2013, Issue 10. Art. No.: CD010309. DOI: 10.1002/14651858.CD010309.pub2.

Copyright (C) 2013 The Cochrane Collaboration. Published by John Wiley \& Sons, Ltd.

\begin{abstract}
A B S T R A C T
Background

The use of combination antiretroviral therapy (cART) comprising three antiretroviral medications from at least two classes of drugs is the current standard treatment for HIV infection in adults and children. Current World Health Organization (WHO) guidelines for antiretroviral therapy recommend early treatment regardless of immunologic thresholds or the clinical condition for all infants (less than one years of age) and children under the age of two years. For children aged two to five years current WHO guidelines recommend (based on low quality evidence) that clinical and immunological thresholds be used to identify those who need to start cART (advanced clinical stage or CD4 counts $\leq 750$ cells $/ \mathrm{mm}^{3}$ or per cent CD $\leq \leq 25 \%$ ). This Cochrane review will inform the current available evidence regarding the optimal time for treatment initiation in children aged two to five years with the goal of informing the revision of WHO 2013 recommendations on when to initiate cART in children.
\end{abstract}

Objectives

To assess the evidence for the optimal time to initiate cART in treatment-naive, HIV-infected children aged 2 to 5 years.

\section{Search methods}

We searched the Cochrane Central Register of Controlled Trials (CENTRAL), MEDLINE, EMBASE, the AEGIS conference database, specific relevant conferences, www.clinicaltrials.gov, the World Health Organization International Clinical Trials Registry platform and reference lists of articles. The date of the most recent search was 30 September 2012.

\section{Selection criteria}

Randomised controlled trials (RCTs) that compared immediate with deferred initiation of cART, and prospective cohort studies which followed children from enrolment to start of cART and on cART.

Optimal time for initiating antiretroviral therapy (ART) in HIV-infected, treatment-naive children aged 2 to 5 years old (Review)

Copyright @ 2013 The Cochrane Collaboration. Published by John Wiley \& Sons, Ltd. 


\section{Data collection and analysis}

Two review authors considered studies for inclusion in the review, assessed the risk of bias, and extracted data on the primary outcome of death from all causes and several secondary outcomes, including incidence of CDC category C and B clinical events and per cent CD4 cells (CD4\%) at study end. For RCTs we calculated relative risks (RR) or mean differences with 95\% confidence intervals (95\% $\mathrm{CI})$. For cohort data, we extracted relative risks with $95 \% \mathrm{CI}$ from adjusted analyses. We combined results from RCTs using a random effects model and examined statistical heterogeneity.

\section{Main results}

Two RCTs in HIV-positive children aged 1 to 12 years were identified. One trial was the pilot study for the larger second trial and both compared initiation of cART regardless of clinical-immunological conditions with deferred initiation until per cent CD4 dropped to $<15 \%$. The two trials were conducted in Thailand, and Thailand and Cambodia, respectively. Unpublished analyses of the 122 children enrolled at ages 2 to 5 years were included in this review. There was one death in the immediate cART group and no deaths in the deferred group (RR 2.9; $95 \%$ CI 0.12 to 68.9). In the subgroup analysis of children aged 24 to 59 months, there was one CDC C event in each group (RR 0.96; 95\% CI 0.06 to 14.87) and 8 and 11 CDC B events in the immediate and deferred groups respectively (RR 0.95; $95 \%$ CI 0.24 to 3.73). In this subgroup, the mean difference in CD4 per cent at study end was 5.9\% (95\% CI 2.7 to 9.1 ). One cohort study from South Africa, which compared the effect of delaying cART for up to 60 days in 573 HIV-positive children starting tuberculosis treatment (median age 3.5 years), was also included. The adjusted hazard ratios for the effect on mortality of delaying ART for more than 60 days was 1.32 (95\% CI 0.55 to 3.16 ).

\section{Authors' conclusions}

This systematic review shows that there is insufficient evidence from clinical trials in support of either early or CD4-guided initiation of ART in HIV-infected children aged 2 to 5 years. Programmatic issues such as the retention in care of children in ART programmes in resource-limited settings will need to be considered when formulating WHO 2013 recommendations.

\section{PLAIN LANGUAGE SUMMARY}

\section{When is the best time to start antiretroviral therapy in children 2 to 5 years old who have HIV infection?}

Antiretroviral combination therapy (cART) has been shown to be effective in slowing down the progression of AIDS and in reducing HIV-related illnesses and death. In infants and children who are diagnosed with HIV infection and are below two years of age the World Health Organization (WHO) recommends that cART should be started immediately. In children aged 2 to 5 years the WHO 2010 recommendations stated that treatment should be started when the body's defence system has started to weaken (as indicated by a decline in a child's CD4 cell count) or complications have occurred. This systematic review was undertaken to help inform the 2013 WHO guidelines which aimed to revise the recommendations of when to start therapy in 2 to 5 years old children. The authors identified two randomised controlled trials (RCTs) that compared immediate with deferred initiation of cART in HIV-positive children aged 1 to 12 years in Thailand or Cambodia. Additional analyses of 122 children enrolled in the two studies at ages 2 to 5 years were made available for this review. A cohort study from South Africa in HIV-positive children (median age 3.5 years) starting tuberculosis treatment and ART was also included. Results showed that we still lack enough evidence to determine whether early or late initiation of cART is best in children aged 2 to 5 years. The authors recognized the lack of evidence but highlighted the potential value of simplifying WHO recommendations to start cART in all children below five years with the goal of providing programmatic advantage to treatment programmes in resource-limited settings.

Optimal time for initiating antiretroviral therapy (ART) in HIV-infected, treatment-naive children aged 2 to 5 years old (Review) 


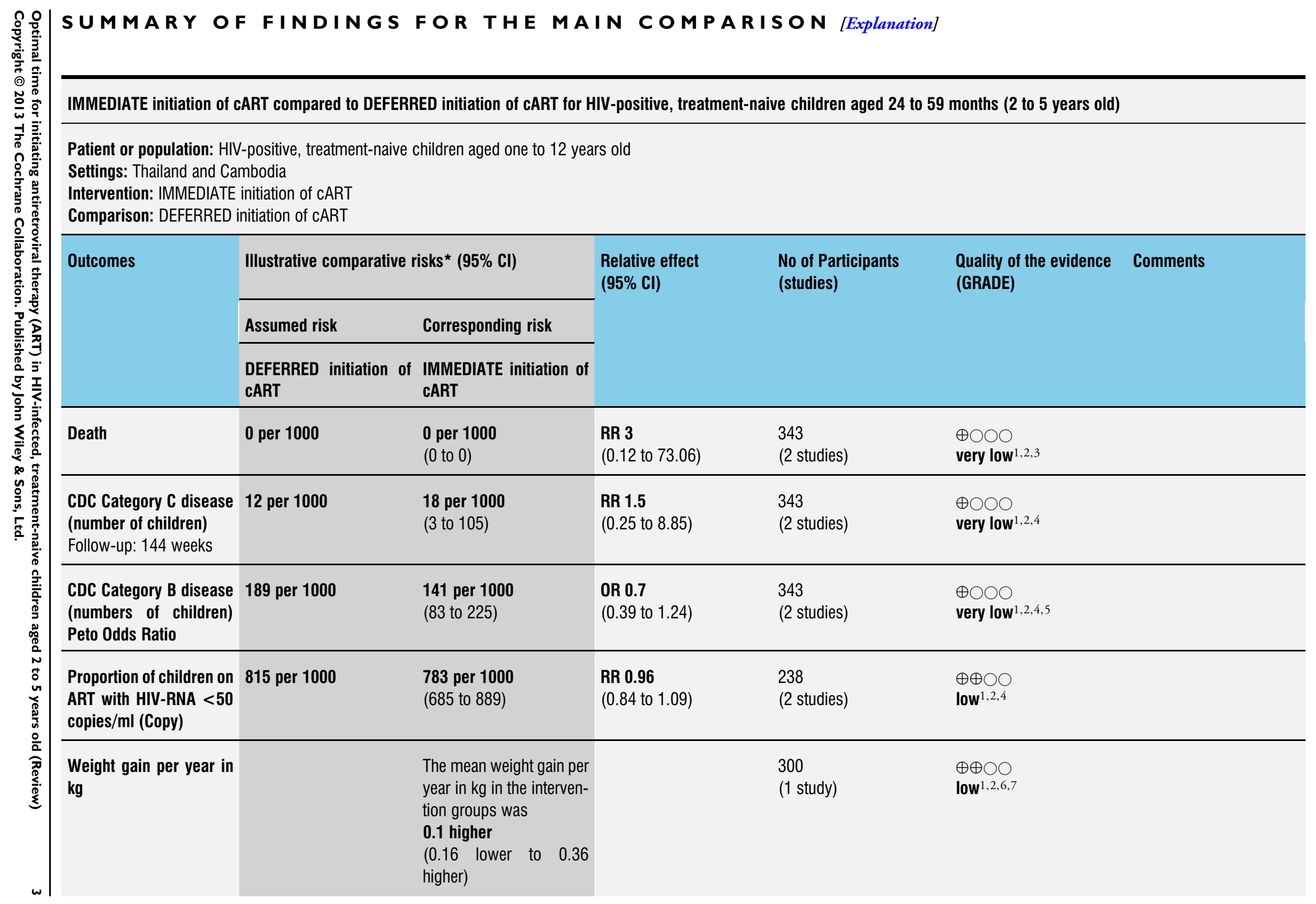




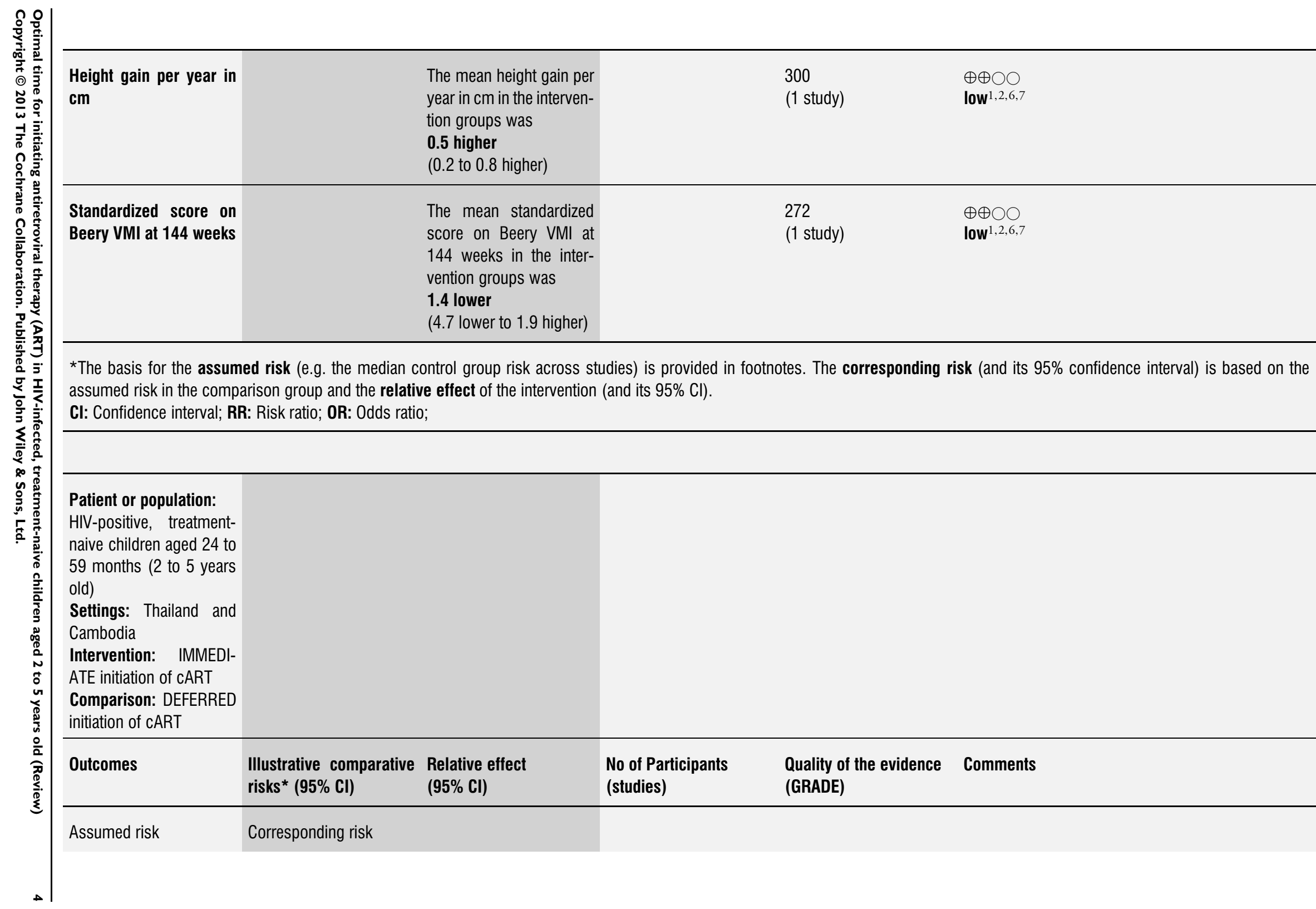




\section{DEFERRED initiation of}

CART

As the trials were open-label neither participants nor caregivers were blinded. However outcome assessors were blinded in the PREDICT

trial. Attrition was low in both trials. Information on the randomisation procedure was lacking in Ananworanich 2008. However, given that this trial is relatively small, we judged the overall risk of bias to be low for the two trials together.

${ }^{2}$ The age group included in the trials ranged from one year to 12 years old and did not focus on the specific population focus of this review: ages 24 to 59 months.

3 The confidence interval is very large and the event rate very low. There was only one death.

${ }^{4}$ The event rate was very low and the overall sample size is also small.

5 The trials reported conflicting results and there was substantial unexplained heterogeneity.

6 The results are from only one trial so consistency cannot be adequately gauged.

The sample size is less than 400 and according to the GRADE approach imprecision is present when continuous outcomes are compared in samples less than 400 . 


\section{B A C K G R O U N D}

\section{Description of the condition}

The World Health Organization (WHO) estimated that in 2011 there were 2.5 million (2.24 to 2.8 million) people who became newly infected with HIV (UNAIDS 2012). Of these, an estimated 330000 [200 000-270 000] were children less than 15 years old. This is a $24 \%$ decrease in global figures since 2009 and can largely be attributed to the expansion in access to services for preventing mother-to-child transmission of HIV, the primary route of HIV acquisition in children.

Sub-Saharan Africa is disproportionately represented in the epidemic with $69 \%$ of the global total of people living with HIV (UNAIDS 2012). An estimated $90 \%$ of the world's children living with HIV live in sub-Saharan Africa. At the end of 2011, 54\% of the 14.2 million people (adults and children) in need of treatment in low- and middle-income countries were receiving antiretroviral therapy (UNAIDS 2012). However, in sub-Saharan Africa, antiretroviral therapy coverage of children in the region is far below the global average, at just $28 \%(25-31 \%)$.

\section{Description of the intervention}

The use of combination antiretroviral therapy (cART) comprising three antiretroviral medications from at least two classes of drugs is the current standard treatment for HIV infection in adults and children (WHO 2010b). While the availability of cART has changed the course of paediatric HIV infection with a notable reduction in mortality observed over long-term follow-up (Patel 2008), late initiation of CART and poor retention in care remain significant challenges in resource-limited settings (Fenner 2010). Determining the optimal timing to initiate cART in children is challenging for several reasons. As children grow, the organs involved in drug metabolism mature. As a result, the pharmacokinetic environment is dynamic and treatment needs to be adjusted according to age and weight (Heidari 2012). If malnutrition is present or treatment for co-infections such as tuberculosis or malaria is required, the pharmacokinetics of drugs may be further altered (Heidari 2012). Markers of disease progression, such as the CD4 count, are unreliable in young children limiting disease monitoring (Prendergast 2012). Given that treatment is life-long, the risk of drug resistance is prolonged and the potential for longterm toxicity exists (Heidari 2012). Determining the optimal time to initiate cART in children is therefore critical.

Current World Health Organization (WHO) guidelines for antiretroviral therapy recommend early treatment regardless of immunologic or clinical thresholds for all infants (less than one year of age) and children under the age of two years (WHO 2010b). The decision to initiate early treatment in infants was based on the evidence provided by the Children with HIV Early Antiretroviral Therapy trial (CHER) (Violari 2008) and supported by a Cochrane review (Penazzato 2012). The subsequent extension of this guideline to children under the age of two years was developed with the goal of improving retention and based on the high risk of disease progression and death and the poor predictive value of immunological markers in this age group (Prendergast 2012).

For children aged 24 to 59 months (two to five years) current WHO guidelines recommend that clinical and immunological thresholds be used to identify those children aged 24 to 59 months who need to start cART (WHO 2010b). Specifically, treatment should be initiated for all HIV-infected children with WHO HIV clinical stages 3 and 4, irrespective of CD4 count. In children aged 24 to 59 months ( 2 to 5 years in age), cART should be initiated when CD 4 counts $<=750$ cells $/ \mathrm{mm} 3$ or $\% \mathrm{CD} 4<=25 \%$, irrespective of the WHO clinical stage. These recommendations are acknowledged to be based on low or very low quality evidence.

\section{Why it is important to do this review}

The World Health Organization (WHO) Antiretroviral Therapy Guideline is the main normative cART tool at global level and is used by regional and national programme managers to develop national treatment guidelines. The first guideline was produced in 2002 (WHO 2002), then revised in 2003 (WHO 2003). In 2006, and then again in 2010, separate sets of recommendations - one for adults and adolescents (WHO 2006a; WHO 2010a) and another for children (WHO 2006b; WHO 2010b) - were published as two guideline documents. In 2011, WHO/UNAIDS established a new initiative, Treatment 2.0, that advocates for the development and expanded use of more simplified, less toxic drug regimens, with high barriers to drug resistance, that require minimal clinical monitoring while maintaining therapeutic efficacy (WHO 2011b). Treatment 2.0 aims to expand access to treatment and applies equally to both adults and children. In this context, revising WHO recommendations will require re-examining the evidence for when to start cART in infants and children. While a completed Cochrane review has focused on the effectiveness of cART in children aged under two years (Penazzato 2012), this Cochrane review will inform the revision of the WHO antiretroviral therapy guidelines specifically for when to initiate cART in children aged two to five years old.

\section{O B JE C T IVES}

To assess the evidence for the optimal time to initiate cART in treatment-naive, HIV-infected children aged 24 to 59 months (2 to 5 years old). 


\section{METHODS}

\section{Criteria for considering studies for this review}

\section{Types of studies}

1. Randomised controlled trials (RCTs)

2. Prospective cohort studies which followed children from enrolment to start of cARTand for at least a median of one year on cART

Non-comparative cohort studies in which children are all commenced on cART at enrolment regardless of clinical-immunological condition were excluded.

\section{Types of participants}

Children with confirmed HIV infection (HIV PCR-positive if diagnosed at age $<18$ months or HIV ELISA-positive if diagnosed after 18 months of age) aged between 24 and 59 months (two to five years in age) who are treatment-naïve (except for exposure to drugs to prevent mother-to-child transmission) at commencement of the study.

Studies which included the above age group within a larger age range were included. We contacted trial authors to obtain the specific age range 24 to 59 months.

Studies in children with tuberculosis and other opportunistic illnesses were included.

Studies focused only on infants (less than one year in age) were excluded. These are included in another Cochrane review (Penazzato 2012).

For RCTs, at study entry, children must not have fulfilled guideline criteria for starting antiretroviral treatment recommended at the time of the study.

\section{Types of interventions}

Studies which compare initiating triple cART at different thresholds.

1. In RCTs, the intervention group must have initiated triple cART irrespective of clinical stage or CD4 count (immediate initiation). The control group must have initiated cART using clinical and immunological criteria to determine the time for initiation (deferred initiation) as recommended at the time of the study.

2. In cohort studies, children starting triple cART at different CD4 percent or CD4 counts must have been compared. Analysis must have included adjustment for time-dependent confounding and lead-time bias specifically to evaluate the effect of timing of cART initiation on outcomes.

\section{Types of outcome measures}

\section{Primary outcomes}

Mortality (all-cause)

\section{Secondary outcomes}

1. Clinical occurrence of new HIV-related events (death or AIDS-defining illness)

2. Time to event of new HIV-related events (death or AIDSdefining illness)

3. Immunological response (change in mean or median CD4+ cell count (mean or median relative change (percent) or mean or median absolute change, compared with baseline, and standard deviation or range as appropriate)

4. Adherence

5. Loss-to-follow-up

6. Virologic response (proportion of patients achieving and maintaining an undetectable viral load, as defined by the investigators; change in HIV-RNA levels (mean relative change (percent) or mean absolute change, compared with baseline, and standard deviations)

7. HIV drug resistance

\section{Adverse events}

Severe adverse events were reported. If classified according to grade 1 to 4 of the Adverse Event Toxicity Scale, we reported grade 3 and 4 events. Using this scale, grade 1 and 2 denote mild to moderate symptoms, grade 3 denote serious symptoms and grade 4 denote life-threatening events requiring significant clinical intervention. Grade 5 denotes death (DAIDS 2009).

\section{Search methods for identification of studies}

See: HIV/AIDS Collaborative Review Group search strategy. The search aimed to be comprehensive and included published and unpublished studies and was not limited to any language.

\section{Electronic searches}

We developed the search strategy with the assistance of the HIV/ AIDS Review Group Trials Search Co-ordinator. We formulated a comprehensive and exhaustive search strategy in an attempt to identify all relevant randomised controlled trials and cohort studies regardless of language or publication status (published, unpublished, in press, and in progress). Full details of the Cochrane HIV/ AIDS Review Group methods and the journals hand-searched are published in the section on Collaborative Review Groups inThe Cochrane Library. 
For the RCT search, we combined the RCT strategy developed by The Cochrane Collaboration and detailed in the Cochrane Handbook for Systematic Reviews of Interventions (Higgins 2009) in combination with terms specific to initiation of antiretroviral therapy and children. The search was iterative and a number of trial searches were run first as there are no database-specific terms for 'initiation' of treatment and so we used many free text terms. This increased the yield and hence the search sensitivity but reduced the precision.

As there are no validated search strategies for cohort studies, our strategy was informed by the cohort strategy developed by BMJ Clinical Evidence (http://clinicalevidence.bmj.com/x/set/static/ ebm/learn/665076.html).

We searched the following databases:

\section{Journal databases}

\section{For RCT Identification:}

- Medline via Pubmed - see search strategy conducted on 27 June 2012 in Appendix 1

- EmBase - see search strategy conducted on 27 June 2012 in Appendix 2

- Cochrane Central Register of Controlled Trials

(CENTRAL) - see search strategy conducted on 27 June 2012 in Appendix 3

\section{For cohort identification:}

- Medline via Pubmed - see search strategy conducted on 11 September 2012 in Appendix 4

- EmBase - see search strategy conducted on 11 September 2012 in Appendix 5

\section{Conference databases:}

We searched the AEGIS database (www.aegis.org) on 24 July 2012. www.aegis.org contained abstracts from the following major related conferences: 1 st-5th International AIDS Society (IAS) Conference on HIV Pathogenesis and Treatment and Prevention (2001-2009); 10th-17th International AIDS Conference (IAC) (1994-2008); 1st-16th Conference of Retrovirus and Opportunistic Infections (CROI) (1994-2009); US National HIV Prevention Conference; 7th-14th British HIV Association (2001-2008); and 8th-9th European AIDS Clinical Society Conference (2001, 2003).

AEGIS does not allow for multiple search strings and so, in order to search the database effectively, a separate search was done for relevant terms combined with the term [random*] to identify relevant trials:
ANTIRETROVIRAL* AND RANDOM* AND (child* OR infant* OR toddler* OR pediatric* OR paediatric*)

The more recent conferences (up to 2012) were covered by searching the conference web-sites of the International AIDS Society, the International AIDS Conference and the CROI. The abstracts of the 1st, 2nd, 3rd and 4th International Workshop on HIV Pediatrics were hand-searched in Reviews in Antiviral Therapy \& Infectious Diseases where they are published following the conference.

\section{Ongoing trials:}

To identify ongoing RCTs we searched ClinicalTrials.gov (http:/ /clinicaltrials.gov/) on 9 July 2012 and the World Health Organization International Clinical Trials Registry Platform (http:// apps.who.int/trialsearch/) on 12 July 2012.

\section{Searching other resources}

We also checked the reference lists of all studies identified by the above methods and examined any systematic reviews, meta-analyses, or guidelines we identified during the search process for references.

We were in close contact with individual researchers working in the field, and policymakers based in inter-governmental organizations including the World Health Organization (WHO) and UNAIDS. We did not conduct hand-searching of specific journals other than those searched by the Cochrane HIV/AIDS Review Group and already included in CENTRAL.

\section{Data collection and analysis}

\section{Selection of studies}

For the RCT search of journal databases, NS and ME read the titles, abstracts and descriptor terms of all downloaded material from the electronic searches to identify potentially eligible reports. Full text articles were be obtained for all citations identified as potentially eligible and NS and ME independently inspected these to establish the relevance of the article according to the pre-specified criteria. Where there was any uncertainty as to the eligibility of the record, we obtained the full article.

NS and ME independently applied the inclusion criteria, and any differences arising were resolved by discussions with the third reviewer, MD. Studies were reviewed for relevance based on study design, types of participants, exposures and outcome measures. For the additional cohort search of journal databases, NS read the titles, abstracts and descriptor terms of all downloaded material from the electronic searches to identify potentially eligible reports. After identification of potentially eligible articles, ME checked these and any uncertainty was discussed and resolved by discussions with the third review author, MD. 
For all conference abstract searching, NS read the titles, abstracts and descriptor terms of all downloaded material and manually hand-searched conference abstract books when it was not possible to search the web-site effectively. NS contacted authors of abstracts to confirm whether the study had been published and/or whether a final report was available. Abstracts which described studies already identified in the electronic searches were linked to these.

\section{Data extraction and management}

NS independently extracted data into a standardised data extraction form. ME checked the data extraction independently. The following characteristics were extracted from each included study.

- Administrative details: Trial or study identification number; author(s); published or unpublished; year of publication; number of studies included in paper; year in which study was conducted; details of other relevant papers cited;

- Details of the study: Study design; type, duration and completeness of follow-up; country and location of study (e.g. higher-income vs. lower-income country); informed consent and ethics approval;

- Details of participants: Setting, numbers, relevant baseline characteristics including CD4 count and viral load;age range;

- Details of intervention: CD4 count and age at which treatment was initiated; drug combinations; additional cointerventions; and

- Details of outcomes: Mortality; HIV-related morbidity; HIV-RNA viral load measurements and proposed levels for suppression, as defined by the authors; clinical disease progression; CD4+ cell counts; adverse events and toxicity.

- Details of the analysis: For RCTs, details of the type of analysis (intention-to-treat or per protocol); for cohort studies, details of the type of adjustment performed in the analysis.

\section{Assessment of risk of bias in included studies}

For both RCTs and cohort studies, NS and ME independently examined the components of each included study for risk of bias using a standard form. This includes information on the sequence generation, allocation concealment, blinding (participants, personnel and outcome assessor), incomplete outcome data, selective outcome reporting and other sources of bias. The methodological components of the trials were assessed and classified as at a high, low or unclear risk of bias as per the Cochrane Handbook for Systematic Reviews of Interventions (Higgins 2009). Where differences arose, these were resolved by discussions with the third reviewer, MD.

Sequence generation

- Adequate: investigators described a random component in the sequence generation process such as the use of random number table, coin tossing, cards or envelops shuffling etc

- Inadequate: investigators described a non-random component in the sequence generation process such as the use of odd or even date of birth, algorithm based on the day/date of birth, hospital or clinic record number

- Unclear: insufficient information to permit judgment of the sequence generation process

\section{Allocation concealment}

- Adequate: participants and the investigators enrolling participants cannot foresee assignment, e.g. central allocation; or sequentially numbered, opaque, sealed envelopes.

- Inadequate: participants and investigators enrolling participants can foresee upcoming assignment, e.g. an open random allocation schedule (e.g. a list of random numbers); or envelopes were unsealed or nonopaque or not sequentially numbered

- Unclear: insufficient information to permit judgment of the allocation concealment or the method not described

\section{Blinding}

- Adequate: blinding of the participants, key study personnel and outcome assessor, and unlikely that the blinding could have been broken. Or lack of blinding unlikely to introduce bias. No blinding in the situation where non-blinding is not likely to introduce bias.

- Inadequate: no blinding, incomplete blinding and the outcome is likely to be influenced by lack of blinding

- Unclear: insufficient information to permit judgment of adequacy or otherwise of the blinding

\section{Incomplete outcome data}

- Adequate: no missing outcome data, reasons for missing outcome data unlikely to be related to true outcome, or missing outcome data balanced in number across groups

- Inadequate: reason for missing outcome data likely to be related to true outcome, with either imbalance in number across groups or reasons for missing data

- Unclear: insufficient reporting of attrition or exclusions

\section{Selective Reporting}

- Adequate: a protocol is available which clearly states the primary outcome as the same as in the final trial report

- Inadequate: the primary outcome differs between the protocol and final trial report

- Unclear: no trial protocol is available or there is insufficient reporting to determine if selective reporting is present

Control of time-dependent confounding (cohort studies only)

The use of standard regression models for the analysis of cohort studies with time-updated measurements may result in biased estimates of treatment effects if time-dependent confounders are present (Robins 2000). In cohorts of HIV-infected patients CD4 cell percentage or CD4 cell counts are measured regularly to assess the patients' eligibility for cART and, once on cART, to monitor therapy. CD4 count is a time-dependent confounder because it predicts both future cART and outcome, and is influenced by past 
antiretroviral therapy. In other words, CD4 count is on the causal pathway between treatment and the outcome.

- Adequate: appropriate methods were used to control for time-dependent confounding (e.g. marginal structural models)

- Inadequate: there was no control for time-dependent confounding

- Unclear: insufficient reporting to determine whether there was control for time-dependent confounding

\section{Other forms of bias}

- Adequate: there is no evidence of bias from other sources

- Inadequate: there is potential bias present from other sources (e.g. early stopping of trial, fraudulent activity, extreme baseline imbalance or bias related to specific study design)

- Unclear: insufficient information to permit judgment of adequacy or otherwise of other forms of bias

\section{Measures of treatment effect}

Data analysis was conducted using (RevMan) version 5.1.7. For RCT data, outcome measures for dichotomous data (e.g. death, virological suppression) were calculated as relative risks with 95\% confidence intervals. For continuous data (e.g. CD4+ cell counts, HIV-RNA viral loads) we calculated the mean difference and standard deviation where means were reported. Where medians and ranges were reported, we extracted the data directly from the report.

For cohort data, we reported on the adjusted analysis using the estimate of effect reported in the study. Where the adjusted estimate of effect was reported with 95\% Confidence Intervals (CI), we calculated the Standard Error in order to enter the data into RevMan, using the following formula for ratio measures: lower limit $=\ln$ (lower confidence limit given for HR) upper limit $=\ln$ (upper confidence limit given for HR) intervention effect estimate $=\ln \mathrm{HR}$ $\mathrm{SE}=$ (upper limit - lower limit) $/ 3.92$

\section{Unit of analysis issues}

We did not anticipate cluster or cross-over trials, with the potential for unit of analysis issues, being conducted to investigate this question.

\section{Dealing with missing data}

Where data was missing or required clarification, we contacted study authors to request additional data. Should this not have been possible, we would have stated explicitly where calculations were based on assumptions regarding missing data.

In trials which included participants from within a larger age range, we contacted study authors to obtain the specific age range 24 to 59 months.

\section{Assessment of heterogeneity}

Where RCTs were found to be methodologically or clinically comparable, we pooled trial results in a meta-analysis. As we anticipated the presence of statistical heterogeneity we combined the data using the random effects model throughout.

Only one cohort study was included in the review, so it was not applicable to assess heterogeneity. In future updates of this review, where cohort studies are found to be methodologically or clinically comparable, we will pool the results in a meta-analysis using the generic inverse variance function in RevMan to allow adjusted data to be used in the analysis. As we anticipate heterogeneity due to the likelihood of different analytical techniques and different adjusted variables, we will combine studies using the random effects model. For the RCT meta-analyses, we formally tested for statistical heterogeneity using the Chi-square test for statistical homogeneity with a $10 \%$ level of significance as the cut-off. The amount of any statistical heterogeneity was quantified using the $\mathrm{I}^{2}$ statistic (Higgins 2002).

Where studies do not have combinable outcomes, we provide the data in a narrative form.

\section{Data synthesis}

For meta-analysis of RCTs, we combined the results and calculated the overall relative risk and 95\% confidence intervals using the Mantel-Haenszel random-effects model. For continuous data, we combined the mean differences to calculate a weighted mean difference and standard deviation, using the inverse-variance random-effects model. Peto fixed-effects models were used in sensitivity analyses. If time-to-event data is available in future updates, we will combine the hazard ratios reported in the RCTs using the inverse-variance random-effects model.

For cohort studies in future updates, we will combine the adjusted estimates of effects using the inverse-variance random-effects model.

\section{Subgroup analysis and investigation of heterogeneity}

We anticipated statistical heterogeneity due to differences between studies conducted in resource-constrained compared with resource-rich settings, and planned to present the results according to these sub-groups should this have been necessary.

We performed subgroup analyses restricted to children less than 5 years old who had a CD4 above $25 \%$ to best inform the current (2012) WHO guideline processes.

\section{Sensitivity analysis}

For RCTs, we planned to explore the effect of study quality on the results by excluding those studies where allocation concealment was unclear or inadequate from the meta-analysis and assessing the effect of this on the overall results. For cohort studies we planned 
to examine the effect of adjustments for confounding and in particular confounding by indication.

We also conducted meta-analyses using the Peto Odds Ratio in addition to the RR for those comparisons where the event rate was extremely low.

\section{GRADE assessment}

GradePro 2008 was used to create Summary of Findings and Evidence Profile tables. GradePro software was developed as part of a larger initiative led by the Grading of Recommendations Assessment, Development and Evaluation (GRADE) Working Group. GRADE offers a system for rating quality of evidence in systematic reviews and guidelines and grading strength of recommendations in guidelines (Guyatt 2011). Use of GradePro within a Cochrane systematic review facilitates the process of presenting and grading evidence transparently (http://ims.cochrane.org/ revman/other-resources/gradepro/about-gradepro).

In determining the level of evidence for each outcome, both the efficacy results and the assessment of the risk of bias was integrated into a final assessment of the level of evidence and full details of the decision was provided in footnotes.

\section{RES U L T S}

\section{Description of studies}

\section{Results of the search}

\section{Electronic databases}

\section{Journal databases}

\section{RCT studies}

Using the RCT string, the PUBMED search yielded 914 records, the EMBASE search yielded 616 records and the CENTRAL search yielded 127 records. After deduplication using ProCite software, we screened 1475 records of which 54 were identified as potentially eligible RCTs or cohort studies and the full texts were obtained. See Figure 1. 
Figure I. Study flow diagram of database yields using the RCT string

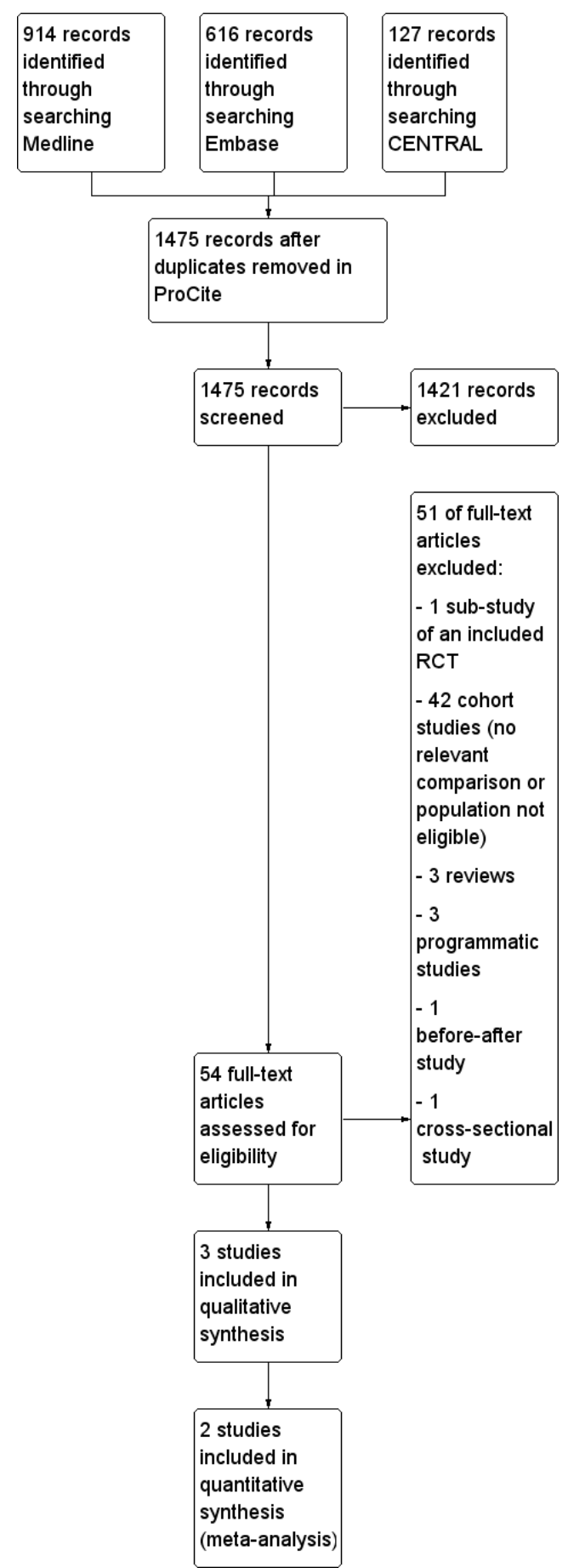

Optimal time for initiating antiretroviral therapy (ART) in HIV-infected, treatment-naive children aged 2 to 5 years old (Review) 


\section{Cohort studies}

Using the additional cohort string, the PUBMED search yielded 130 records, and the EMBASE search yielded 950 records. These were de-duplicated electronically using EndNote reference management software, and 33 duplicate records were removed prior to manual checking of the remaining 1047 records. No studies in addition to those already selected for full text review in the RCT string search, were identified.

\section{Conference databases}

The www.aegis.com conference database search yielded 170 records of which 9 abstracts were selected for further assessment. We also searched the conference web-sites for the following specific conferences using a broad term 'child':

- International AIDS Society 2009: 305 records identified of which 16 were selected for further assessment

- International AIDS Society 2011:513 records identified of which 15 were selected for further assessment

- International AIDS Conference 2012: 115 records

identified of which three were selected for further assessment; all relevant sessions reviewed and no additional relevant records identified

- CROI 2011: 69 records identified of which four were selected for further assessment

- CROI 2012: 85 records identified of which four were selected for further assessment

NS identified one study (Munyagwa 2012) from the conference searches which was published after the date the electronic journal database search. This study was excluded after an eligibility assessment of the full text. No other studies identified in the conference searching were in addition to those studies already reviewed in the electronic database searches, or contained data which was relevant to this review after complete data sets were obtained. We contacted a total of five abstract authors to confirm whether their abstracts contained the same data as that reported in publications.

\section{Trials Registries}

We searched ClinicalTrials.gov (http://clinicaltrials.gov/) on 9 July 2012 and retrieved 300 records of which 9 studies were selected for further assessment.

We also searched the World Health Organization International Clinical Trials Registry Platform (http://apps.who.int/ trialsearch/) on 12 July 2012 and retrieved 12 records of which one study was selected for further assessment.

None of the 21 studies assessed met our inclusion criteria. in future updates we will capture the details of studies meeting our inclusion criteria under Characteristics of ongoing studies.

\section{Searching other resources}

To supplement the electronic searching for conference databases, we searched the following books of conference abstracts manually during August and September 2012:

- CROI 2009: 2 records were identified for further assessment

- CROI 2010: 2 records were identified for further assessment

- 1st International Workshop on HIV Pediatrics (157 abstracts in total): 13 records identified for further assessment

- 2nd International Workshop on HIV Pediatrics (123 abstracts in total); 10 records identified for further assessment

- 3rd International Workshop on HIV Pediatrics (186 abstracts in total): 10 records identified for further assessment

- 4th International Workshop on HIV Pediatrics (200 abstracts in total); 5 records identified for further assessment

Through discussions with experts in the field and prior discussions at conferences, we were aware of the PREDICT 2012 trial which was published during the conduct of this review. This study was reported in several of the above abstracts which we selected for further assessment. No additional eligible studies were identified in this way.

\section{Included studies}

\section{RCTs}

Following detailed eligibility assessment, two RCTs were identified as eligible (Ananworanich 2008; PREDICT 2012) from the database searches. Additional searches of conferences and the trials registries identified further abstracts which referred to these trials, but no additional RCTs were identified in this way.

Details of the included studies are reported in the table Characteristics of included studies.

In summary, two RCTs have evaluated the timing of initiating cART in HIV-positive children aged 1 to 12 years, with CD4\% between 15 and 24\%, and no CDC clinical stage C disease. cART was initiated immediately on enrolment (IMMEDIATE group) or deferred until the CD4\% dropped to < 15\% (DEFERRED group). The first trial (Ananworanich 2008) commenced in December 2001 and was designed as a small feasibility study prior to the conduct of the larger trial which commenced in March 2006 ( PREDICT 2012). Both trials were conducted in south-east Asia: Ananworanich 2008 was conducted in Thailand with a sample size of 43 and PREDICT 2012 was conducted in Thailand and Cambodia with a sample size of 300 .

Of note, no trials have been conducted only in the specific age group this review covers, viz. 24 to 59 months. In the Ananworanich 2008 trial, the median age of participants was 4.8 years (IQR: 2.7 to 6.6) and in the PREDICT 2012 trial, 59\% 
$(88 / 150)$ and $55 \%(83 / 150)$ of children were aged 1 to 6 years in the IMIMEDIATE and DEFERRED group respectively.

\section{Cohorts}

Following detailed eligibility assessment, several cohort studies were selected for further assessment of the full-text. After discussions between the co-authors, only one cohort study was deemed eligible (Yotebieng 2010). See the details of the study in the table Characteristics of included studies.

This study was an observational cohort of prospectively collected routine data of children infected with tuberculosis (TB) and compared the timing of initiation of cART using the number of days since TB treatment was initiated, rather than based on CD4 cell count or percentage. The cohort study commenced in April 2004 and took place in a paediatric clinic attached to a tertiary hospital in Soweto, South Africa.
The study included 573 children aged 1 to 15 years with a median age of 3.5 years (IQR: $1.4-6.8$ ). The median length of followup was 9.6 months (IQR: 1.9 to 23.1) which was less than the median follow-up of one year stipulated in our review criteria for study eligibility. However, after discussion among the co-authors, we determined that given the paucity of data on ART initiation in TB populations, we would include the study in the current review.

\section{Excluded studies}

After assessing the full-text articles 51 studies were excluded for the reasons detailed in Figure 1.

\section{Risk of bias in included studies}

The detail of the risk of bias are presented in the Characteristics of included studies and the graphical summary of the risk of bias is represented in Figure 2; Figure 3.

Figure 2. Risk of bias graph: review authors' judgements about each risk of bias item presented as percentages across all included studies.

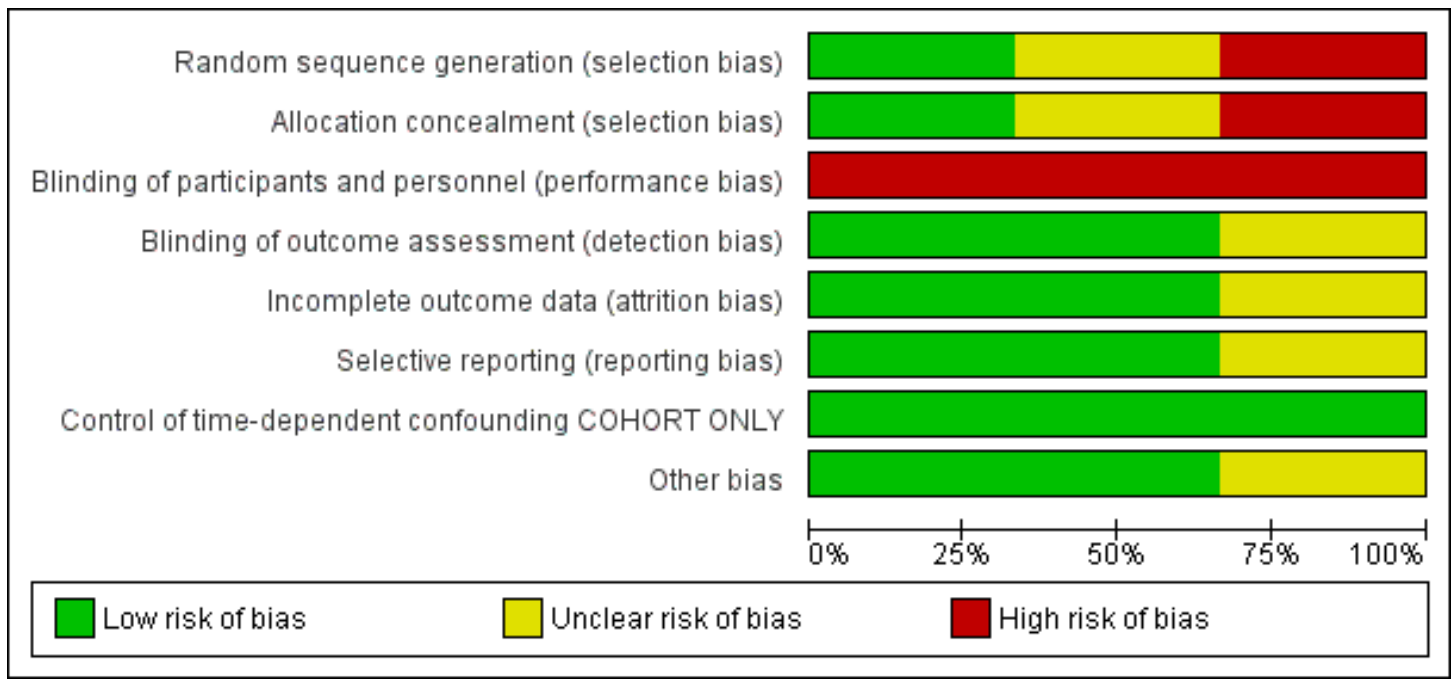

Optimal time for initiating antiretroviral therapy (ART) in HIV-infected, treatment-naive children aged 2 to 5 years old (Review) 
Figure 3. Risk of bias summary: review authors' judgements about each risk of bias item for each included study.

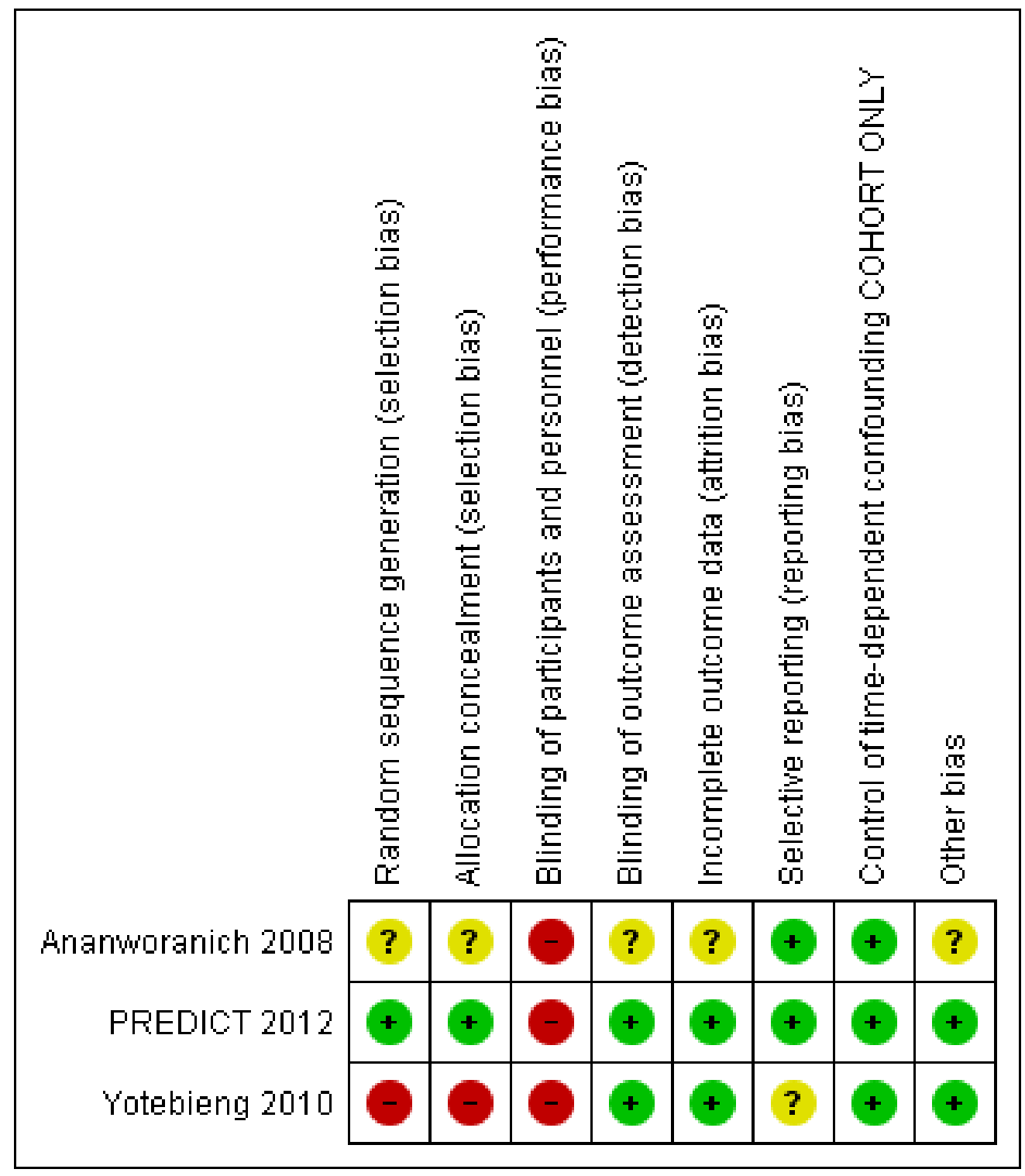




\section{Allocation}

\section{RCTs}

In Ananworanich 2008 we were unable to assess the risk of bias in the randomisation and allocation procedures due to a lack of reporting and judged the risk of bias as unclear. We judged the risk of bias due to selection bias as low for the PREDICT 2012 trial as the random sequence was generated by a computer and allocation was done centrally.

\section{Cohort}

As Yotebieng 2010 is a cohort study, allocation was not done randomly. The study is therefore at high risk of selection bias but it was analysed in a way that will have reduced bias (mimicking a randomised trial - see other sources of bias below).

\section{Blinding}

\section{RCTs}

Both trials were open-label and thus neither participants and their caregivers nor personnel were blinded, potentially introducing a high risk of performance bias.

In the Ananworanich 2008 trial we were not able to determine whether outcomes were assessed in a blinded manner and we rated the risk of bias as unclear. In PREDICT 2012, the primary outcomes were assessed by an independent committee blinded to allocation and the risk of detection bias was low.

\section{Cohort}

The personnel and caregivers were not blinded to whether the children were receiving CART or not and we judged the risk of performance bias as high.

Although the authors do not report on blinding for the two primary outcomes of death and virological suppression (in those patients receiving CART) or on how death was confirmed, we judged the lack of blinding as unlikely to result in a high risk of detection bias given the nature of these outcomes

\section{Incomplete outcome data}

\section{RCTs}

Both trials had high follow-up rates $(\leq 10 \%)$ and risk of attrition bias was low.

\section{Cohort}

The overall loss to follow-up was $13 \%$ and the study used timeto-event analysis to address this. The authors also report that the children lost to follow-up did not differ from those remaining in care in any of the baseline characteristics or timing of ART initiation and we judged the risk of bias to be low.

\section{Selective reporting}

\section{RCTs}

We were able to compare the protocol in www.clinicaltrials.gov with the PREDICT 2012 report and no selective reporting was noted with a low risk of bias. We were not able to identify the protocol for Ananworanich 2008 and rated the risk of bias as unclear.

\section{Cohort}

We were not able to compare the protocol with the results and rated the risk of bias as unclear.

\section{Other potential sources of bias}

\section{RCTs}

We did not identify other potential sources of bias in either trial.

\section{Cohort}

For cohort studies we assessed whether the analysis had appropriately controlled for time-dependent confounding. In the Yotebieng 2010 cohort the authors made use of inverse probability-of-treatment and censoring (IPTC) weighting of marginal structural models, an appropriate statistical analysis to control for time-dependent confounding, which mimics a randomised trial. We rated the risk as low.

\section{Effects of interventions}

See: Summary of findings for the main comparison IMMEDIATE initiation of cART compared to DEFERRED initiation of cART for HIV-positive, treatment-naive children aged one to 12 years old; Summary of findings 2 Subgroup analysis: IMMEDIATE initiation of cART compared to DEFERRED initiation of cART for HIV-positive, treatmentnaive children aged 24 to 59 months ( 2 to 5 years old) 
We present the outcomes as these were reported in the study reports and include any additional outcomes where these were not included in our outcomes list in the protocol. As the two trials were conducted in similar populations we did not consider clinical or methodological heterogeneity as barriers to meta-analysis. Therefore we have combined the results from both trials in metaanalyses using the random effects model where possible. Where indicated, we conducted further sensitivity analyses using different analytical models. We report the results from our RevMan calculations and provide narrative results where it was not possible to extract the data for further analysis e.g. where adjusted analyses were conducted or where medians were reported.

The authors of both trials provided unpublished age-specific data for the 24 to 59 month age group for outcomes determined to be most critical for guideline development, and we present these results as a post hoc subgroup analysis and report on this in the text.

For the results from the cohort study, we present the adjusted and weighted results and not the crude results due to the inherent confounding present in unadjusted analyses.

\section{Mortality}

In the PREDICT 2012 trial, one death was observed in the IMMEDIATE group . Due to the low number of events the results are unlikely to be meaningful (see Analysis 1.1). This death was in the IMMEDIATE group in the 24 to 59 month age group. See Analysis 2.1.

In the Yotebieng 2010 cohort study, death was compared in three different time periods: 1 ) cART initiation after 15 days following TB treatment compared with within 15 days; 2) cART initiation after 30 days (1 month) following TB treatment compared with within 30 days ( 1 month); and 3) cART initiation after 60 days following TB treatment compared within 60 days.

- More than 15 days vs less than 15 days:

- The authors calculated a weighted HR accounting for time-dependent level of immuno-suppression, viral load, weightfor-age $\mathrm{Z}$ score and age at TB treatment initiation. The reported weighted HR was 0.82 (95\% CI: 0.48, 1.41). See Analysis 3.1. (note that the calculated 95\% CI in RevMan differs very slightly from the $95 \%$ CI reported by the authors).

- More than 30 days vs less than 30 days:

- The authors calculated a weighted HR of $0.86(95 \%$ CI: 0.46, 1.60). Weighting accounted for time-dependent level of immuno-suppression, viral load, weight-for-age $\mathrm{Z}$ score and age at TB treatment initiation. See Analysis 3.1 (note that the calculated $95 \% \mathrm{CI}$ in RevMan differs very slightly from the reported $95 \% \mathrm{CI}$ ).

- More than 60 days vs less than 60 days:

- The authors calculated a weighted HR of 1.32 (95\% CI: $0.55,3.16)$. Weighting accounted for time-dependent level of immuno-suppression, viral load, weight-for-age $\mathrm{Z}$ score and age at TB treatment initiation. See Analysis 3.1 (note that the calculated $95 \%$ CI in RevMan differs very slightly from the reported $95 \% \mathrm{CI}$ ).

From Analysis 3.1 a dose-response gradient can be observed in the increasing HR values. This indicates that deferring cART initiation to later than 15 days after commencing $\mathrm{TB}$ treatment was protective against death but this effect was reduced at 30 days. Deferred initiation became potentially harmful if cART was deferred beyond 60 days. None of these HR were statistically significantly different from 1.

NOTE: The denominators in Analysis 3.1 vary with each cut-off time period due to censoring (the authors provide an example of a child whose follow-up ended 25 days after TB initiation and prior to cART who would be classified for the 15 days cut-off but not for the 30 or 60 day cut-off periods).

\section{Morbidity}

\section{AIDS-free survival}

In the PREDICT 2012 trial, the authors report an intention-totreat analysis of AIDS-free survival rates for all children at week 144 using the Kaplan-Meier product limit method and the IMMEDIATE group as the reference group. At week 144 AIDS-free survival rates in intention to treat analysis were $98.7 \%$ (95\%CI: 94.7, 99.7\%) in the DEFERRED group and $97.9 \%$ (95\% CI: 93.7, 99.3\%) in the IMMEDIATE group (Log rank $\mathrm{p}=0.6$ ). In per protocol analysis, AIDS-free survival rates at week 144 were 98.7\% (95\% CI: 94.7, 99.7\%) in the DEFERRED group and 98.6\% (95\% CI 94.5-99.6\%) in the IMMEDIATE group (Log rank $\mathrm{p}=0.96)$.

\section{CDC category $C$ disease}

No children in the Ananworanich 2008 trial developed CDC Category C disease. In the PREDICT 2012 trial three children in the IMMEDIATE group developed CDC Category C disease (Pneumocystis jiroveci pneumonia (1); Pneumonia/Sepsis leading to death (1); Disseminated Penicilliosis (1)) and two children developed Category $\mathrm{C}$ disease in the DEFERRED group (Oesaphageal Candidiasis (1); Extrapulmonary Tuberculosis (1)). We calculated the relative risk (RR) in RevMan (See Analysis 1.2). The RR was 1.50 (95\% CI: 0.25, 8.85; $\mathrm{p}=0.65$ ). The authors report CDC category $\mathrm{C}$ events incidence as 4.9 per 1000 person-years of followup (PYFU) in the DEFERRED group and 7.6 per 1000 PYFU in the IMMEDIATE group (unadjusted hazard ratio $(\mathrm{HR})=0.7$ (95\%CI: $0.1,3.9 ; \mathrm{p}=0.6)$ and $\mathrm{HR}$ adjusted for age and gender $=$ 0.8 (95\%CI: $0.1,4.6 ; \mathrm{p}=0.8$ ).

In the subgroup analysis of the 24 to 59 month age group in the PREDICT 2012 trial, the RR was 0.96 (95\% CI: 0.06, 14.87; p $=0.98)$. See Analysis 2.2. 


\section{CDC Category B disease}

We combined the data from both trials for children in all age groups using the random effects model. The RR was $1.42(95 \%$ CI: 0.14, 14.28; $\mathrm{p}=0.77$; see Analysis 1.3). We categorised the pulmonary TB observed in the Ananworanich 2008 trial as CDC Category $\mathrm{B}$ disease. The results show that there was a greater likelihood of CDC Category B diseases in the IMMEDIATE group than in the DEFERRED group but that this was not statistically significant. However, statistical heterogeneity was present $\left(\mathrm{Chi}^{2}=\right.$ $2.84, \mathrm{df}=1 ; \mathrm{p}=0.09)$ with the $\mathrm{I}^{2}=65 \%$ indicating that there was substantial heterogeneity not explained by chance.

We conducted a sensitivity analysis and re-analysed the data using the Peto Odds Ratio (POR) because it is the recommended approach for uncommon events, particularly when there are trials with zero events as in the Ananworanich 2008 trial. The POR was 0.70 (95\% CI: 0.39, 1.24; p = 0.22; see Analysis 1.4). Note that the weighting for Ananworanich 2008 changes from 33.5\% to $7.8 \%$ in this analysis. These results indicate that the odds of developing CDC Category B events was 0.7 times lower in the IMMEDIATE group than in the DEFERRED group. However, heterogeneity was present $\left(\mathrm{Chi}^{2}=5.22, \mathrm{df}=1 ; \mathrm{p}=0.02\right)$ with an $\mathrm{I}^{2}=81 \%$.

The high level of heterogeneity would suggest that these results should not be combined in a meta-analysis, although the second analysis which down-weights the Ananworanich 2008 trial is probably more appropriate. The authors of PREDICT 2012 report that CDC class B incidence was 110 (95\%CI: 80, 147) events per 1000 PYFU in the DEFERRED group and 88 (95\%CI: 61,122) events per $1000 \mathrm{PYFU}$ in the IMMEDIATE group (incidence rate ratio $($ IRR $)=1.25 ; 95 \%$ CI: $0.8,1.9 ; \mathrm{p}=0.3$ ). Therefore, early treatment of 45 children for a year rather than deferring treatment until CD4 falls to below $15 \%$ would prevent 1 CDC category B event. This result is more consistent with that of Analysis 1.4 (note the reference or control group is the IMMEDIATE group in the results reported from the PREDICT 2012 trial, and in Analysis 1.4 the reference group is the DEFERRED group).

In the meta-analysis of the subgroups of 24 to 59 month age groups, we performed the identical sensitivity analysis to that reported above. The result was similar to that of all age groups (POR $=0.76 ; 95 \%$ CI: 0.29, 2.02; p = 0.59). See Analysis 2.4.

\section{Pulmonary TB}

The incidence of pulmonary TB was low in both trials. We combined the results for children of all age groups in a random effects meta-analysis (see Analysis 1.5). The risk of developing pulmonary TB was greater in the IMMEDIATE group than the DEFERRED group but the difference was not statistically significant $(\mathrm{RR}=$ 3.21, 95\% CI: $0.52,19.89 ; \mathrm{p}=0.21$ ). Heterogeneity was minimal $\left(\mathrm{Chi}^{2}=0.99, \mathrm{df}=1 ; \mathrm{p}=0.32\right)$; with an $\mathrm{I}^{2}=0 \%$ indicating no important statistical heterogeneity.
Given the low number of events we again conducted a sensitivity analysis using the Peto Odds Ratio and found similar results (POR: 3.49, 95\% CI: $0.67,18.13 ; \mathrm{p}=0.14)$ with likely unimportant heterogeneity $\left(\mathrm{Chi}^{2}=1.20, \mathrm{df}=1 ; \mathrm{p}=0.27 ; \mathrm{I}^{2}=17 \%\right)$. The authors of the Ananworanich 2008 trial report that the four TB events were not considered to be the result of immune reconstitution syndromes due to the large gap between ART initiation and TB diagnosis (median time of 60 weeks with range 48 to 72 weeks). All four children responded well to anti-TB treatment.

In the meta-analysis of the subgroups of 24 to 59 month age groups, one case of pulmonary $\mathrm{TB}$ was observed in each group $(\mathrm{RR}=1.19 ; 95 \% \mathrm{CI}: 0.19,7.27 ; \mathrm{p}=0.85)$ with low heterogeneity $\left(\mathrm{Chi}^{2}=1.36, \mathrm{df}=1 ; \mathrm{p}=0.24 ; \mathrm{I}^{2}=26 \%\right)$.

\section{Median time before development of CDC B or C events}

Both trials reported on the median time before development of either CDC B or C events for children of all age groups. In the Ananworanich 2008 trial, events developed in the IMMEDIATE group after a median of 60 (IQR: 48 - 72) weeks and no events developed in the DEFERRED group. In the PREDICT 2012 trial, events developed after a median of 9 (IQR: 4 - 30) weeks since enrolment in the IMMEDIATE group compared with a median of 57 (IQR: 34 - 101) weeks in the DEFERRED group.

\section{Virological and immunological response}

\section{Virological suppression}

\section{Proportion of children on cART with HIV-RNA $<50$ copies/ml}

Both trials provided data on the proportion of children of all age groups on cART with HIV-RNA $<50$ copies $/ \mathrm{ml}$, but the PREDICT 2012 trial restricted analyses to children on first-line cART for at least 48 weeks whereas this was not the case in the Ananworanich 2008 trial so we cannot be certain that the time periods are identical. We combined the data in a random effects meta-analysis (RR $=0.96$; $(95 \% \mathrm{CI}: 0.84,1.09 ; \mathrm{p}=0.52$; see Analysis 1.7). From these data it is evident that the probability of a viral load $<50$ copies $/ \mathrm{ml}$ was similar in the IMMEDIATE group and the DEFERRED group. There was no statistical heterogeneity $\left(\mathrm{Chi}^{2}=0.29, \mathrm{df}=1 ; \mathrm{p}=0.59 ; \mathrm{I}^{2}=0 \%\right)$.

In the subgroup analysis of the 24 to 59 month age group in the PREDICT 2012 trial, the results were similar to that for all age groups $(\mathrm{RR}=1.11 ; 95 \% \mathrm{CI}: 0.86,1.43 ; \mathrm{p}=0.43)$. 


\section{Proportion of children on cART with HIV-RNA $<400$ copies/ml}

The Yotebieng 2010 cohort study assessed virologic suppression in 70\% (324/461) of children who initiated cART and who had at least one viral load measurement after cART initiation. The results were analysed in the same three time-periods as for mortality.

- More than 15 days vs less than 15 days:

- The authors calculated an adjusted HR which accounted for baseline differences. The reported adjusted HR was 0.98 (95\% CI: 0.76, 1.26; see Analysis 3.2).

- More than 30 days vs less than 30 days:

- The authors calculated a HR adjusted for baseline

differences of 0.95 (95\% CI: 0.73, 1.23; see Analysis 3.2).

- More than 60 days vs less than 60 days:

- The authors calculated an adjusted HR of $0.84(95 \%$

CI: 0.61, 1.15; see Analysis 3.2).

As for the analysis of death (see Analysis 3.1) we noted a doseresponse gradient in the hazard ratios for virologic suppression: children were less likely to achieve virologic suppression for each increment in deferred time to cART initiation, however, none of the HRs were statistically significantly different from 1.

\section{Median viral load at study end}

The authors of the Ananworanich 2008 trial report on the median viral load at the end of the study (median of 134 weeks follow-up) for children of all age groups. In the IMMEDIATE group, there was a statistically significantly lower median viral load of $1.7 \log _{10}$ copies/ml (IQR 1.7 - 2.5) compared with the DEFERRED group viral load of $3.1 \log _{10}$ copies/ml (IQR $1.7-4.5 ; \mathrm{p}=0.039$ ). The median change in viral load at study end was $-2.8 \log _{10}$ copies $/ \mathrm{ml}$ (IQR -3.4 to -1.9) in the IMMEDIATE group compared with - 1.8 $\log _{10}$ copies/ml (IQR: -3.2 to -0.3 ). The $\mathrm{p}$ value for this difference was $\mathrm{p}=0.079$.

\section{Median CD4\% at study end}

For children of all age groups, the authors of Ananworanich 2008 reported that at the end of the study (median of 134 weeks followup), the median CD $4 \%$ in the IMMEDIATE group was statistically significantly higher at 31\% (IQR: 24 - 39) compared with the DEFERRED group of median CD4\% 23\% (IQR: 17 - 31; p $=0.032$ ). The median CD $4 \%$ change was $13.5 \%$ (IQR: 4 - 18) in the IMMEDIATE group compared with $3 \%$ (IQR: -2 to 13 ) in the DEFERRED group $(\mathrm{p}=0.012)$.

\section{Mean CD4\% and change in CD4\% at week I 44}

In the PREDICT 2012 trial, the authors report a mean CD4\% at week 144 of $33.2 \%$ (SD: 6.4) in the IMMEDIATE group compared with a mean CD $4 \%$ of $24.8 \%$ (SD: 7.4 ) in the DEFERRED group for children of all age groups. We calculated the mean difference (see Analysis 1.9) as 8.40\% (95\% CI: 6.83, 9.97; $\mathrm{p}<0.00001)$. At the end of the study the mean CD4\% in the IMMEDIATE group was thus higher than in the DEFERRED group. The authors also conducted a Kaplan-Meier analysis to determine the probability of achieving CD $4 \% \geq 30 \%$ at week 96 . The probability was 0.83 (95\% CI $0.77-0.89)$ in the IMMEDIATE group and 0.74 (95\% CI 0.62-0.84) in the DEFERRED group ( $\mathrm{p}<0.001)$ indicating that the children in the IMMEDIATE group were more likely to achieve CD $4 \% \geq 30 \%$ at week 96 . In the subgroup analysis of the 24 to 59 month age group in the PREDICT 2012 trial, the mean CD4\% at the end of the trial was $5.9 \%$ higher in the IMMEDIATE group than in the DEFERRED group (95\%CI: 2.74, 9.06; $\mathrm{p}=0.0003$; see Analysis 2.7).

\section{Proportion of children with CD4\% $<15 \%$ at study end}

In Ananworanich 2008 no children in the IMMEDIATE group had a CD $4 \%<15 \%$ at study end and three children did in the DEFERRED group - all of whom had less than four week of cART. In PREDICT 2012, using the Last Observation Carried Forwards (LOCF) approach, one child in the IMMEDIATE group and nine children in the DEFERRED group had a CD $4 \%<15 \%$ at study end (unpublished data). The meta-analysis of the combined data showed that fewer children in the IMMEDIATE group had CD $4 \%<15 \%$ at study end compared with in the DEFERRED group ( $R R=0.11 ; 95 \% \mathrm{CI}: 0.02,0.60 ; \mathrm{p}=0.01)$. There was no statistical heterogeneity (see Analysis 1.10). In the 24 to 59 month age group, the proportion of children with CD $4 \%<15 \%$ in the IMMEDIATE group was lower than that in the DEFERRED group but the results were less precise and no longer statistically significant $(\mathrm{RR}=0.40 ; 95 \% \mathrm{CI}: 0.06,2.52 ; \mathrm{p}=0.33$, see Analysis 2.8).

\section{Growth parameters}

\section{Weight}

In the PREDICT 2012 trial, for all age groups, the mean weight gain per year was $2.2 \mathrm{~kg}$ (SD: 1.1) in the IMMEDIATE group and $2.1 \mathrm{~kg}$ (SD: 1.2 ) in the DEFERRED group, for a mean difference of $0.1 \mathrm{~kg}$ (95\% CI: -0.16, 0.36; $\mathrm{p}=0.45$; see Analysis 1.11 ). Median weight-for-age $\mathrm{z}$ scores at study end were available for all children in both trials. In the Ananworanich 2008 trial, the score in the IMMEDIATE group was -1.1 (IQR: -1.5 to -0.8) and in the DEFERRED group the score was -1.0 (IQR: -1.7 to 0.2 ). In the PREDICT 2012 trial, the score was -1.27 (IQR: -1.78 to 0.39 ) in the IMMEDIATE group and -1.40 (IQR: -1.99 to -0.89 ) in the DEFERRED group. These scores indicate that growth was compromised in both groups in both trials (see Analysis 1.12). The change in median weight-for-age $\mathrm{z}$ score was calculated in PREDICT 2012. In the IMMEDIATE group, the change in median score was 0.26 (IQR: -2.00 to -0.75 ) which was a greater gain 
in weight than the median change in score of 0.04 (IQR: -0.28 to 0.34 ) in the DEFERRED group ( $\mathrm{p}=0.02$ for difference). In a multivariate model, the authors found that the effect of group allocation remained significant $(\mathrm{p}=0.01)$ after adjusting for baseline CD $4 \%(<20 \%$ and $\geq 20 \%)$, age $(<5$ years and $\geq 5$ years $)$, haemoglobin level, and HIV RNA level. In the analysis of the 24 to 59 month age group in the PREDICT 2012 trial, weight gain was similar in the IMMEDIATE and DEFERRED groups, with a mean difference of $-0.03 \mathrm{~kg}$ (95\% CI: $-0.25,0.19 ; \mathrm{p}=0.79$, see Analysis 2.9).

\section{Height}

In the PREDICT 2012 trial, for all ages, the mean height gain per year was $5.4 \mathrm{~cm}$ (SD: 1.4$)$ in the IMMEDIATE group and $4.9 \mathrm{~cm}$ (SD: 1.2$)$ in the DEFERRED group, for a mean difference of $0.50 \mathrm{~cm}$ (95\% CI: 0.20, 0.80; $\mathrm{p}=0.0009)$; see Analysis 1.13). Median height-for-age $\mathrm{z}$ scores at study end were available in both trials. In the Ananworanich 2008 trial, the score in the IMMEDIATE group was -1.4 (IQR: -2.0 to -0.8) and in the DEFERRED group the score was -0.8 (IQR: -1.3 to -0.4 ). In the PREDICT 2012 trial, the score was -1.50 (IQR: -2.35 to -0.54 ) in the IMMEDIATE group and -1.73 (IQR: -2.42 to -0.95 ) in the DEFERRED group. These scores indicate that height was compromised in both groups in both trials (see Analysis 1.14). The change in median height-for-age $\mathrm{z}$ score was calculated in the PREDICT 2012 trial. In the IMMEDIATE group, the change in median score was 0.25 (IQR: -0.21 to -0.69) in the IMMEDIATE group and the median change in score was -0.02 (IQR: -0.40 to 0.34 ) in the DEFERRED group ( $\mathrm{p}=0.004$ for difference). The children in the IMMEDIATE group thus gained more height than those in the DEFERRED group. In an adjusted analysis, the authors found that the effect of group allocation remained significant $(\mathrm{p}=0.002)$ after adjusting for baseline CD4\% $(<20 \%$ and $\geq 20 \%)$, age $(<5$ years and $\geq 5$ years), haemoglobin level, and HIV RNA level. In the analysis of the 24 to 59 month age group in PREDICT 2012, mean height gain was slightly greater in the IMMEDIATE group compared to the DEFERRED group, with a mean difference of $0.3 \mathrm{~cm}$ (95\%CI: -0.28, 0.88; $\mathrm{p}=0.31$; see Analysis 2.10).

\section{Neurological parameters}

In the PREDICT 2012 trial, the neurodevelopment sub-study was phased in after the main study had started and baseline Beery Visual Motor Integration assessment was only performed in 71 children. The mean baseline scores for children of all age groups in the IMMEDIATE and DEFERRED groups were 81.5 (SD: 17.2) and 87.1 (SD: 14.4) respectively. There was no statistically significant difference between the groups at study end (week 144). The mean difference was -1.40 (95\% CI: $-4.70,1.90$; $\mathrm{p}=0.41$; see Analysis 1.15). The authors report that for the 71 children who had a baseline assessment, the mean difference in Beery standard score change from baseline from week 0 to 144 between IMMEDIATE and DEFERRED arms was $-4.37(-12 \cdot 00$ to $3 \cdot 26$; $p=0 \cdot 26)$. In the subgroup analysis, the Beery Visual Motor Integration score was available for 42 and 40 of the children aged 24 to 59 months in the IMMEDIATE and DEFERRED group respectively. Again, there was no statistically significant difference between the groups at study end. The mean difference was 2.3 (95\% CI: $-4.37,8.97$; $\mathrm{p}=0.50$; see Analysis 2.11).

\section{Adverse events}

The Ananworanich 2008 trial reported on the proportion of children of all age groups with adverse events, but did not provide data on the severity of the events. All children in the DEFERRED group and $92 \%$ of those in the IMMEDIATE group experienced an adverse event $(R R=0.92 ; 95 \%$ CI: $0.80,1.07 ; p=0.29)$. Both trials reported on the proportion of children of all age groups with adverse events related to cART. Children in the IMMEDIATE group were more likely to develop cART-related adverse events than those in the DEFERRED group but this was not statistically significant $(\mathrm{RR}=1.87 ; 95 \% \mathrm{CI}: 0.77,4.51 ; \mathrm{p}=0.17)$ and there was little statistical heterogeneity $\left(\mathrm{Chi}^{2}=0.96, \mathrm{df}=1 ; \mathrm{p}=0.33\right.$; $\left.\mathrm{I}^{2}=0 \%\right)$. The proportion of children in the 24 to 59 month age group in the PREDICT 2012 trial with ART-related Grade 3 or 4 events was lower in the IMMEDIATE group than in the DEFERRED group $(\mathrm{RR}=0.48 ; 95 \% \mathrm{CI}: 0.04,5.11 ; \mathrm{p}=0.54$; see Analysis 2.12).

\section{GRADE ASSESSMENTS}

GRADE assessments were conducted for all outcomes where data was available to enter into GRADEPro. For the Summary of Findings tables we selected seven outcomes per comparison based on what is most important to patients.

Overall, the quality of evidence observed in the two trials was low or very low. For evidence for initiation of cART in all age groups, we down-graded the evidence for indirectness (as the age group was not specific to the population group of interest) and for serious or very serious imprecision. The PREDICT 2012 trial was well-conducted with the sample size planned to be powered for the primary outcome of AIDS-free survival; however, due to the low number of events and slow disease progression, the study was ultimately under-powered to adequately assess this outcome. Note that we did not create a Summary of Findings Table for the outcome of AIDS-free survival. For all other outcomes, the sample size was considered small and for most outcomes the event rate was extremely small, leading to imprecision in the results. This remained when combined with the data from the Ananworanich 2008 trial as the trial added only 43 children to the total. See Summary of findings for the main comparison.

For the post hoc subgroup analysis of ages 24 to 59 months, we did not downgrade for indirectness as the population was specific 
to the question of this review. However, as this was a post hoc analysis, the risk of selection bias resulted in downgrading for risk of bias. We continued to downgrade for very serious imprecision due to the small sample size, the low event rate and wide confidence intervals. See Summary of findings 2 .

For the evidence arising from the observational study (Yotebieng 2010), the quality of evidence was rated as very low for all outcomes. We downgraded for risk of bias due to a residual risk of selection bias: we cannot be certain that the IPTC weighting of marginal structural models removed all bias. As the study included children aged one to 15 years we downgraded for indirectness. Due to the low number of events, we downgraded for serious imprecision.

Using the GRADE approach, we are very uncertain of the effect estimates overall. 


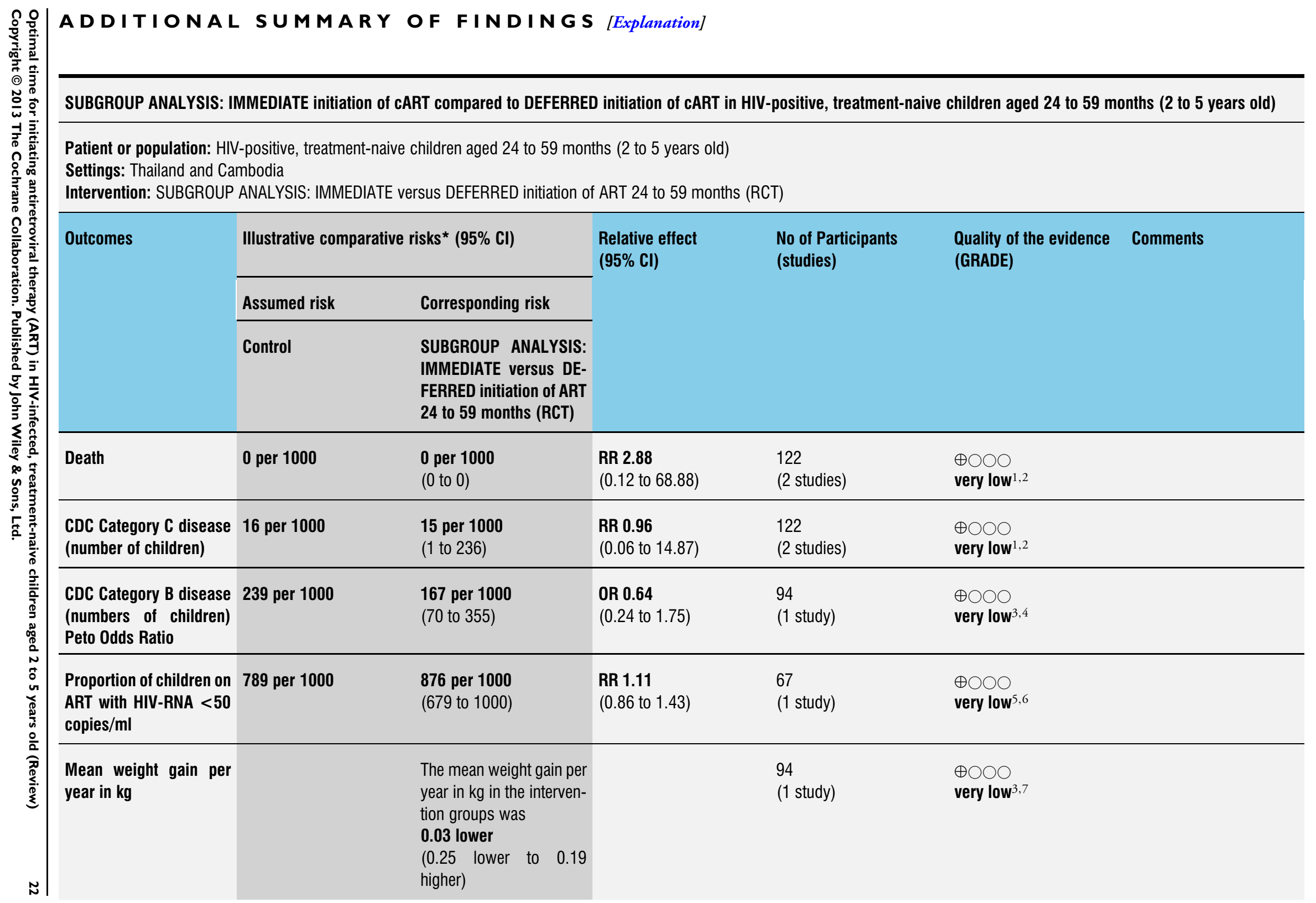




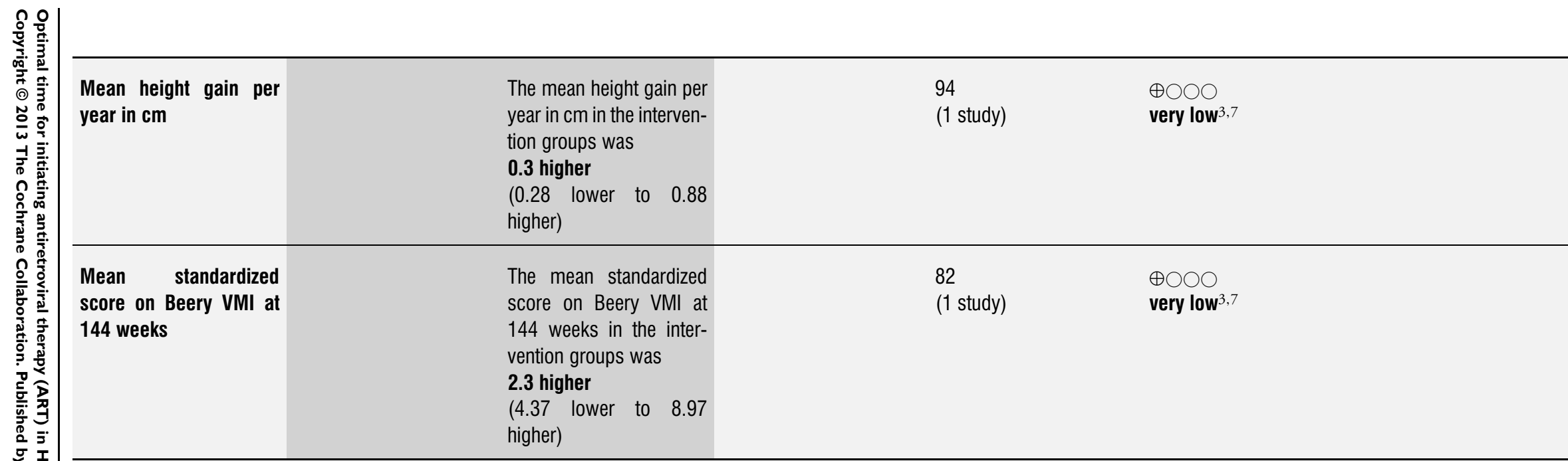

*The basis for the assumed risk (e.g. the median control group risk across studies) is provided in footnotes. The corresponding risk (and its $95 \%$ confidence interval) is based on the assumed risk in the comparison group and the relative effect of the intervention (and its $95 \% \mathrm{Cl}$ ).

Cl: Confidence interval; RR: Risk ratio; OR: Odds ratio;

GRADE Working Group grades of evidence

High quality: Further research is very unlikely to change our confidence in the estimate of effect.

Moderate quality: Further research is likely to have an important impact on our confidence in the estimate of effect and may change the estimate.

Low quality: Further research is very likely to have an important impact on our confidence in the estimate of effect and is likely to change the estimate.

Very low quality: We are very uncertain about the estimate.

${ }^{1}$ This is a subgroup analysis within each of the Ananworanich 2009 and PREDICT 2012 trials. Randomisation was not conducted within

the sub-group. For Ananworanich 2008, the proportion of children aged 24 to 59 months in the IMMEDIATE group was 46\% (11/24)

and in the DEFERRED group it was $89 \%$ (17/19). This differential could introduce selection bias.

${ }^{2}$ The sample size of the two subgroups of age-specific data for 24 to 59 months is 122 and the event rate is very small. The confidence interval is very large.

${ }^{3}$ Randomisation was not conducted within the sub-group. In the PREDICT trial, the proportion of the overall sample in the subgroup in the IMMEDIATE group was $32 \%(48 / 150)$ and in the DEFERRED group it was $31 \%(46 / 150)$. Although this is balanced, we cannot exclude the possibility of selection bias as this analysis was conducted post hoc.

(4) The sample size of the subgroup of age-specific data for 24 to 59 months in the PREDICT 2012 trial is 94 and the event rate is very small. The confidence interval is large.

${ }^{5}$ Randomisation was not conducted within the sub-group. In the PREDICT trial, the proportion of the overall sample in the subgroup $\approx$

in the IMMEDIATE group was 32\% (48/150) and in the DEFERRED group it was 31\% (46/150). In this comparison (children on ART 


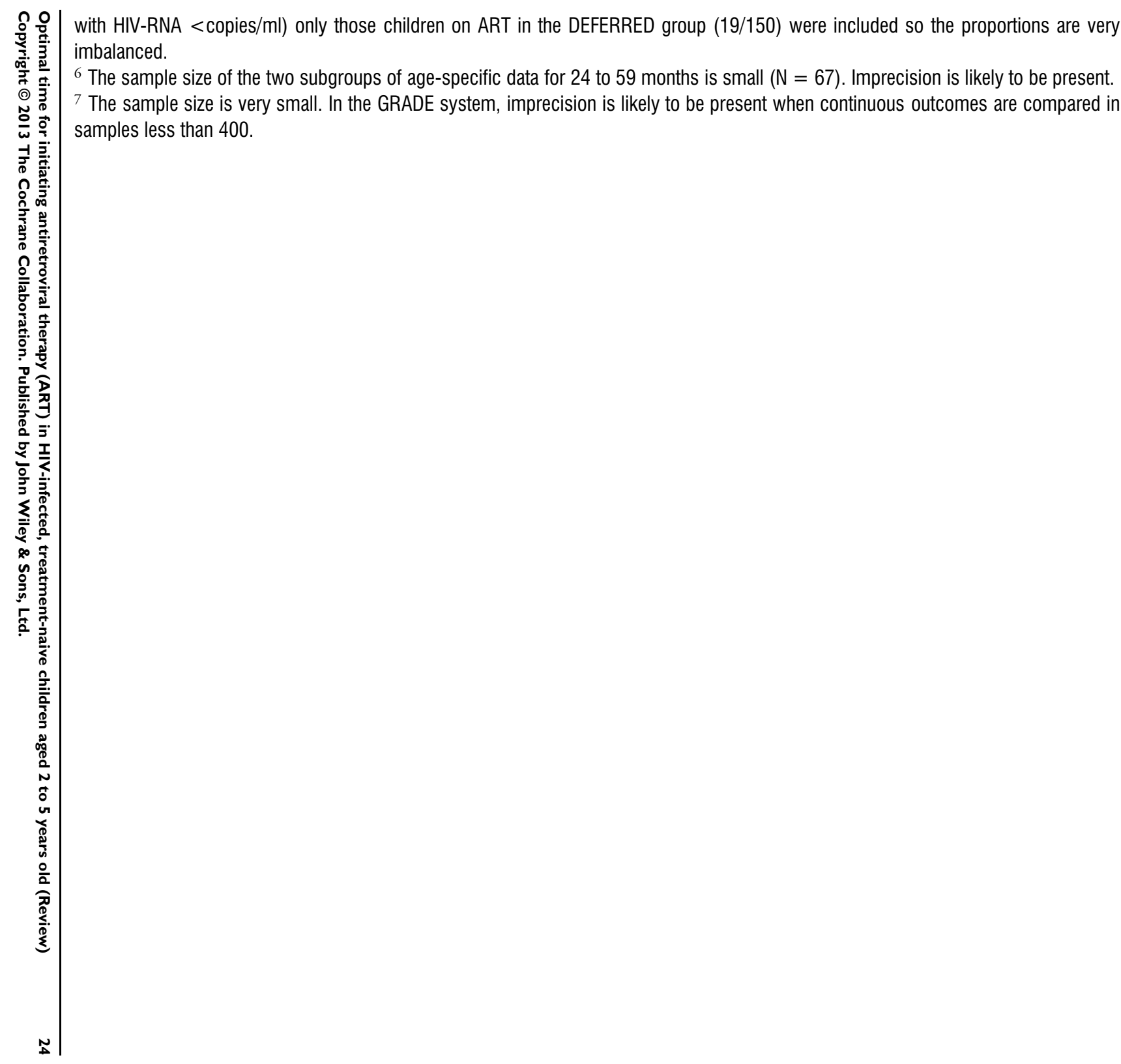




\section{ISCUSSIO N}

\section{Summary of main results}

Our review identified limited evidence from two small randomised controlled trials evaluating the effects of initiating cART in children aged one to 12 years immediately at CD4\% between 15 and $25 \%$, compared with deferring cART until pre-defined immunological and clinical thresholds. No differences in outcomes were noted between the randomised groups.

We identified a single cohort study which compared delaying cART in HIV-infected children co-infected with TB with initiating cART soon after commencing TB treatment. Delaying cART initiation to later than 15 days after commencing TB treatment was protective against death but this effect was reduced at 30 days, and potentially harmful if cART was deferred beyond 60 days.

None of the studies exclusively included children within the specific focus age of two to five years.

\section{Overall completeness and applicability of evidence}

The trials included in this review did not evaluate the effects of immediate or deferred initiation of cART in children aged two to five years, but in the broader age group of one to 12 years. Although we were able to obtain age-specific data from the two trials, the evidence generated from these post hoc sub-group analyses is less robust given that randomisation did not take place within the age-specific sub-group. Stronger evidence would be gained from replicating trials within the specific age group, but it is unlikely that new trials will assess this in future. Antiretroviral treatment has been indicated for all HIV-positive infants and children less than two years of age since 2010 (WHO 2010b). As cART becomes increasingly available across the world, identification and recruitment into trials of children aged two or more may no longer be feasible.

The evidence generated from the two trials and the single included cohort study arise from resource-constrained settings affected by the HIV/AIDS epidemic (Thailand, Cambodia and South Africa). The participants in the trials are therefore likely to be representative of HIV-infected children living in poverty. However, the children and their carers were from urban settings and in this respect the results may not be generalizable to those children and their carers living in impoverished rural areas.

\section{Quality of the evidence}

The quality of the methodological conduct for the larger PREDICT 2012 trial was judged to be high and the risk of bias was likely to be low to moderate for both trials overall. However, when using the GRADE approach to assess the overall quality of the evidence generated in the review, the quality of the evidence was rated as low or very low. This was due to the indirectness of the included population and the marked imprecision present in the results. Imprecision was driven largely by the small sample size and low event rates. The sample size of the PREDICT 2012 trial was powered for the primary outcome of AIDS-free survival; however, due to the low number of events and slow disease progression, the study was ultimately under-powered to adequately assess this and this reduced the quality of the evidence.

In the Yotebieng 2010 cohort study, close attention was paid to confounding and the authors addressed this via weighted adjusted analyses which included adjusting for time-dependent level of immuno-suppression, viral load, weight-for-age $\mathrm{Z}$ score and age at TB treatment initiation. Despite this, the quality of evidence generated by a single cohort study was appraised in GRADE as providing very low evidence due to selection bias (lack of randomisation), indirectness and imprecision.

\section{Potential biases in the review process}

Possible selection biases in the review process were minimised by using a comprehensive search strategy to identify studies and, wherever possible, independently selecting and appraising the studies. In addition to searching journal and conference electronic databases, we conducted hand-searching of several important conference abstract books, including the International Workshop on HIV Pediatrics and recent relevant conferences not yet included in the electronic databases. We contacted several authors of conference abstracts to confirm whether the data in their abstract corresponded to subsequent journal articles or to assess whether the reported data was eligible for inclusion in this review. it is unlikely that important trials have been missed given the high-profile nature of the topic and the close partnership established with agencies and organizations working in this area.

Data extraction was done independently by one author and then checked by a second author. Ideally all data extraction should be conducted independently by two authors; however, this was not feasible given the time constraints of the rapid review process. The results were presented at a WHO Guidelines Committee Meeting in December 2012 providing a further check of the data by experts and investigators familiar with the data, thereby reducing any potential measurement bias introduced by a single author conducting data extraction.

We conducted several sensitivity analyses using different statistical models and estimates of effect. This was especially important to do for several of the analyses which were limited by small denominators and few events (Higgins 2009). Conducting sensitivity analyses allowed us to interrogate the data closely and consider the robustness of the findings. 


\section{Agreements and disagreements with other studies or reviews}

We were unable to identify any other studies or reviews addressing the question of when to start cART in children aged 24 to 59 months. Lewis et al monitored 127 children enrolled in the Paediatric European Network for the Treatment of AIDS 5 and found that initiation of cART at higher CD4 counts may improve long term immune reconstitution and may potentially reduce the so-called 'non-AIDS' morbidity developed as a result of immune activation without causing harm or significantly increasing drugrelated toxicity (Lewis 2011).

\section{AUTHORS, CONCLUSIONS}

\section{Implications for practice}

There is lack of sufficient evidence from clinical trials to assess whether immediate versus deferred initiation of cART in children aged between 2 and 5 years provides clinical and immunological benefits.

While individual level benefits are still unclear, from a programmatic point of view starting cART in all children below 5 years of age may facilitate a more rapid scale-up of paediatric treatment in resource-limited settings where coverage remains low. Removing the $\mathrm{CD} 4$ barrier to treatment initiation for children less than five years is likely to result in prompter initiation of cART for those eligible children whose treatment is delayed while they wait for CD4 tests and results. In these settings it will nevertheless be critical to prioritise cART to those children with a higher risk of mortality where early ART initiation has proven mortality benefit, such as infants less than one year of age and those with advanced clinical and immunological disease.

Based on the evidence in this review, in settings with wide access to CD4 monitoring, good cART coverage and adequate programme retention, CD4-guided initiation of cART should not be viewed as sub-optimal standard of care. Adequate pre-cART clinical followup should be strengthened to ensure prompt recognition of disease progression as soon as this may occur.

\section{Implications for research}

Large randomised trials assessing morbidity and mortality as well as clinical and immunological outcomes with adequate follow-up are warranted to provide the evidence base for effective decision making on when to start cART in children aged 24 to 59 months in resource-limited settings.

\section{ACKNOWLEDGEMENTS}

We are grateful to Joy Oliver, Trial Search Coordinator, for developing the search strategy and for conducting the searches. We thank Tara Hovarth, Managing Editor, for her guidance, especially regarding conference searching, and to Gavrilah Wells for the administrative support.

\section{REF ER E N C E S}

\section{References to studies included in this review}

Ananworanich 2008 \{published data only\}

* Ananworanich J, Kosalaraksa P, Siangphoe U, Engchanil C, Pancharoen C, Lumbiganon P, et al.A feasibility study of immediate versus deferred antiretroviral therapy in children with HIV infection. AIDS research and therapy 2008;5:24. [PUBMED: 18957095]

PREDICT 2012 \{published and unpublished data\}

* Wongsawat J, Puthanakit T, Kanjanavanit S,

Hansudewechakul R, Ngampiyaskul C, Kerr SJ, et al.CD4 cell count criteria to determine when to initiate antiretroviral therapy in human immunodeficiency virusinfected children. The Pediatric infectious disease journal 2010;29(10):966-8. [PUBMED: 20418798]

Yotebieng 2010 \{published data only\}

* Yotebieng M, Van Rie A, Moultrie H, Cole SR, Adimora

A, Behets F, et al.Effect on mortality and virological response of delaying antiretroviral therapy initiation in children receiving tuberculosis treatment. AIDS (London, England) 2010;24(9):1341-9. [PUBMED: 20559039]

\section{References to studies excluded from this review}

Edmonds 2009 \{published data only\}

Edmonds A, Lusiama J, Napravnik S, Kitetele F, Van Rie A, Behets F. Anti-retroviral therapy reduces incident tuberculosis in HIV-infected children. International journal of epidemiology 2009;38(6):1612-21. [PUBMED: 19448046]

Edmonds 2011 \{published data only\}

Edmonds A, Yotebieng M, Lusiama J, Matumona Y, Kitetele F, Napravnik S, et al.The effect of highly active antiretroviral therapy on the survival of HIV-infected children in a resource-deprived setting: a cohort study. PLoS medicine 2011;8(6):e1001044. [PUBMED: 21695087]

Munyagwa 2012 \{published data only\} Munyagwa M, Baisley K, Levin J, Brian M, Grosskurth $\mathrm{H}$, Maher D. Mortality of HIV-infected and uninfected children in a longitudinal cohort in rural south-west 
Uganda during 8 years of follow-up. Tropical medicine \& international health : TM \& IH 2012;17(7):836-43. [PUBMED: 22591447]

\section{Musoke 2010 \{published data only\}}

Musoke PM, Mudiope P, Barlow-Mosha LN, Ajuna P, Bagenda D, Mubiru MM, et al.Growth, immune and viral responses in HIV infected African children receiving highly active antiretroviral therapy: a prospective cohort study. BMC pediatrics 2010;10:56. [PUBMED: 20691045]

Patel 2008 a \{published data only\}

Patel K, Hernán MA, Williams PL, Seeger JD, McIntosh K, Van Dyke RB, Seage GR 3rd, Pediatric AIDS Clinical Trials Group 219/219C Study Team. Long-term effects of highly active antiretroviral therapy on CD4+ cell evolution among children and adolescents infected with HIV: 5 years and counting. Clin Infect Dis 2008;46(11):1751-60. [PUBMED: 18426371]

\section{Patel 2008 b \{published data only\}}

Patel K, Hernan MA, Williams PL, Seeger JD, McIntosh K, Van Dyke RB, Seage GR 3rd, Pediatric AIDS Clinical Trials Group 219/219C Study Team. Long-term effectiveness of highly active antiretroviral therapy on the survival of children and adolescents with HIV infection: a 10-year follow-up study. Clinical infectious Diseases 2008;46(4): 507-15. [PUBMED: 18199042]

\section{Additional references}

\section{DAIDS 2009}

Division of AIDS. Table for grading the severity of Adult and Pediatric Adverse Events. Accessed 28 October 2009 http: //rcc.tech-res.com/Document/safetyandpharmacovigilance/ DAIDS 'AE ' GradingTable 'Clarification 'August2009' Final.pdf.

Fenner 2010

Fenner L, Brinkhof MW, Keiser O, Weigel R, Cornell M, Moultrie H, Prozesky H, Technau K, Eley B, Vaz P, Pascoe M, Giddy J, Van Cutsem G, Wood R, Egger M, Davies MA. Early mortality and loss to follow-up in HIV-infected children starting antiretroviral therapy in Southern Africa. $J$ Acquir Immune Defic Syndr 2010;54(5):542-532.

\section{GradePro 2008}

Jan Bozek, Andrew Oxman, Olger Schunemann. GradePro 2008. 3.2 for Windows. GRADE Working Group, 2008, 2008.

\section{Guyatt 2011}

Guyatt G, Oxman AD, Akl EA, Kunz R, Vist G, Brozek J, et al.GRADE guidelines: 1 . Introduction-GRADE evidence profiles and summary of findings tables. Journal of clinical epidemiology 2011;64(4):383-94. [PUBMED: 21195583]

\section{Heidari 2012}

Heidari S, Mofenson LM, Hobbs CV, Cotton MF, Marlink R, Katabira E. Unresolved antiretroviral treatment management issues in HIV-infected children. Journal of acquired immune deficiency syndromes (1999) 2012;59(2): 161-9. [PUBMED: 22138766]

\section{Higgins 2002}

Higgins JP, Thompson SG. Quantifying heterogeneity in a meta-analysis. Statistics in Medicine 2002;21(11):1539-58.

\section{Higgins 2009}

Higgins JPT, Green S (editors). Cochrane Handbook for Systematic Reviews of Interventions Version 5.0.2 [updated September 2009]. The Cochrane Collaboration, 2009. Available from www.cochrane-handbook.org.

Lewis 2011

Lewis J, Walker AS, Castro H, De Rossi A, Gibb DM, Giaquinto C, Klein N, Callard R. Age and CD4 Count at Initiation of Antiretroviral Therapy in HIV-Infected Children: Effects on Long-term T-Cell Reconstitution. J Infect Dis 2011;205:548-56.

\section{Patel 2008}

Patel K, Hernan MA, Williams PL, Seeger JD, McIntosh $\mathrm{K}$, Van Dyke RB, et al.Long-term effectiveness of highly active antiretroviral therapy on the survival of children and adolescents with HIV infection: a 10-year follow-up study. Clinical infectious diseases : an official publication of the Infectious Diseases Society of America 2008;46(4):507-15. [PUBMED: 18199042]

\section{Penazzato 2012}

Penazzato M, Prendergast A, Tierney J, Cotton M, Gibb D. Effectiveness of antiretroviral therapy in HIV-infected children under 2 years of age. Cochrane database of systematic reviews (Online) 2012;7:CD004772. [PUBMED: 22786492]

Prendergast 2012

Prendergast AJ, Penazzato M, Cotton M, Musoke P, Mulenga V, Abrams EJ, Gibb DM. Treatment of Young Children with HIV Infection: Using Evidence to Inform Policymakers. PLoS Medicine 2012;9(7):e1001273. doi: 10.1371/journal.pmed.1001273.

\section{Robins 2000}

Robins JM, Hernan MA, Brumback B. Marginal structural models and causal inference in epidemiology. Epidemiology 2000;11(5):550-560.

\section{UNAIDS 2012}

Joint United Nations Programme on HIV/AIDS (UNAIDS). Global report: UNAIDS report on the global AIDS epidemic 2012. http://www.unaids.org/en/medial unaids/contentassets/documents/epidemiology/2012/gr2012/ 20121120 UNAIDS 'Global'Report'2012 'en.pdf 2012; Accessed 24 May 2013.

\section{Violari 2008}

Violari A, Cotton MF, Gibb DM, Babiker AG, Steyn J, Madhi SA, Jean-Philippe P, McIntyre JA, CHER Study Team. Early antiretroviral therapy and mortality among HIV-infected infants. N EngJ Med 2008;359:2233-2244.

\section{WHO 2002}

World Health Organization. Scaling up antiretroviral therapy In resource-limited settings: guidelines for a public health approach. 2002; Vol. http://www.who.int/hiv/pub/ prev' care/en/ScalingUp E.pdf (Accessed 29 July 2012). 


\section{WHO 2003}

World Health Organization. Scaling up antiretroviral therapy in resource-limited settings:treatment guidelines for a public health approach [2003 revision]. 2003; Vol. http:/ /www.who.int/hiv/pub/prev" care/en/arvrevision2003en.pdf (Accessed 29 July 2012)

\section{WHO 2006a}

World Health Organization. Antiretroviral therapy for HIV infection in adults and adolescents: recommendations for a public health approach [2006 revision]. World Health Organization, 2006; Vol. http://www.who.int/hiv/pub/ guidelines/artadultguidelines.pdf (Accessed 29 July 2012).

\section{WHO 2006b}

World Health Organization. Antiretroviral therapy of HIV infection in infants and children: towards universal access. Recommendations for a public health approach. 2006; Vol. http://www.who.int/hiv/pub/guidelines/ paediatric020907.pdf (Accessed 29 July 2012).

\section{WHO 2010a}

World Health Organization. Antiretroviral therapy for HIV infection in adults and adolescents: recommendations for a public health approach [2010 revision]. 2010;
Vol. http://whqlibdoc.who.int/publications/2010/

9789241599764 eng.pdf (Accessed 29 July 2012).

\section{WHO 2010b}

World Health Organization. Antiretroviral therapy of HIV infection in infants and children: towards universal access: recommendations for a public health approach [2010 revision]. 2010; Vol. http://whqlibdoc.who.int/ publications/2010/9789241599801 eng.pdf (Accessed 29 July 2012).

\section{WHO 2011a}

World Health Organization. Global HIV/AIDS response: epidemic update and health sector progress towards universal access: progress report 2011. 2011; Vol. http://whqlibdoc.who.int/publications/2011/ 9789241502986 eng.pdf (Accessed 2 August 2012).

\section{WHO 2011b}

World Health Organization. The treatment 2.0 framework for action: catalysing the next phase of treatment, care and support. 2011; Vol. http://whqlibdoc.who.int/ publications/2011/9789241501934'eng.pdf (Accessed 2 August 2012).

* Indicates the major publication for the study 


\section{CHARACTERISTICS OF STUDIES}

\section{Characteristics of included studies [ordered by study ID]}

\section{Ananworanich 2008}

Methods

Participants

\section{STUDY TYPE:}

- Randomised controlled trial

COUNTRY:

- Thailand

SETTING:

- Research clinical sites: The HIV Netherlands Australia Thailand Research

Collaboration/Chulalongkorn University in Bangkok and the Khon Kaen University in Northeast Thailand

DURATION OF RECRUITMENT:

- Dec 2001 - Mar 2003 (Bangkok); Oct 2002 - Mar 2003 (Khon Kaen) DURATION OF TRIAL:

- 3 years and 3 months. Completed March 2005

FOLLOW-UP:

- Length of follow-up was 108 weeks.

- Children were followed monthly for the first three months and then every three months.

- At baseline, CD4 was tested by flow cytometry and CBC and alanine transferase (ALT) were tested.

- At every visit, CD4, Complete Blood Count and ALT were tested.

- At every 24 week visit, viral load (Roche Amplicor Ultrasensitive assay), fasting lipids and glucose were tested.

- Median duration of follow-up from randomization: 134 (IQR: 123 - 154) weeks This trial was a pilot study was conducted to explore the feasibility and HIV disease outcome of the immediate versus deferred strategy as ground work for the PREDICT trial (PREDICT 2012).

\section{INCLUSION CRITERIA:}

- Children aged one to 12 years old, with HIV infection, with CDC clinical stage A or $\mathrm{B}$ and who had never received ART other than zidovudine as part of PMTCT. EXCLUSION CRITERIA:

- Children younger than one year.

- Children with CDC C or CD4 < 15\%

- Children without symptoms or with normal CD4 (> 25\%)

Number of participants randomised: 43

- Median age at randomization: IMMEDIATE group: 5.2 (IQR: 2.4 - 8.0) years;

DEFERRED group: 4.4 (IQR: 2.7 - 5.8) years

- Gender distribution (Male: Female) (n, \%): IMMEDIATE group:10:14 (42: 58); DEFERRED group: 7: 12 (37: 63)

- Median weight for age z-scores (WAZ) (IQR): IMMEDIATE group: -1.0 (-1.5 $0.4)$; DEFERRED group: $-0.1(-1.5-0.3)$

- Median height for age z-scores (WAZ) (IQR): IMMEDIATE group: -1.7 (-2.0 $0.9)$; DEFERRED group: $-0.8(-1.7-0.1)$

- Median percent CD4 count (\%; IQR): IMMEDIATE group: 19 (16 - 22);

DEFERRED group: 20 (17 - 22) 
- Median CD4 count (cells/mm³ ;QR): IMMEDIATE group: 649 (509 - 834); DEFERRED group: 615 (544-818)

All characteristics and baseline data did not differ between the two groups except median triglyceride at 48 weeks. This was statistically significantly higher in the DEFERRED group (98; IQR: 70 - 148) compared with the IMMEDIATE group (69: IQR: 54 - 88) $(\mathrm{p}=0.016)$

Interventions

Outcomes

\section{INTERVENTION: IMMEDIATE GROUP}

- Participants were started on ART immediately at study entry. CONTROL: DEFERRED GROUP

- Participants were started on ART when CD4 fell to $<15 \%$ in those with baseline CD4 20 - 24\% or CD4 dropped by 25\% in those with baseline CD4 $15-19 \%$. A repeat confirmatory CD4 was done immediately if CD4 fell below ART initiation threshold.

ART comprised standard doses of generic individual zidovudine, lamivudine and nevirapine according to the Thai Government Pharmaceutical Organization guidelines COMPLIANCE:

No formal means of assessing compliance is reported.

CO-INTERVENTIONS:

Cotrimoxazole was started immediately with the first decrease of CD4 below 15\% and was continued for at least three months until two consecutive CD4 were above $15 \%$

\section{PRIMARY OUTCOMES:}

- Recruitment rate

- Adherence to randomized group

- Retention in the study

SECONDARY OUTCOMES:

- Proportion (\%) children with CDC C or with CD4 < 15\%

- Growth

- Median CD4\%

- Median Viral load

- ART savings (reduced time on ART)

- ART-related Adverse Events (measured using the 1994 Adult and Pediatric Grading Tables of the Division of AIDS, NIH (DAIDS 1994)

$\begin{array}{ll}\text { Notes } & \text { ETHICS } \\ \text { Institutional Review Board at Chulalongkorn and Khon Kaen Universities } \\ \text { INFORMED CONSENT: } \\ \text { All caregivers gave signed informed consent. } \\ \text { FUNDING } \\ \text { Not specifically reported. The Thai Government Pharmaceutical Organization provided } \\ \text { anti-retrovirals }\end{array}$

Risk of bias

\section{Bias}

Authors' judgement

Random sequence generation (selection Unclear risk bias)

Not reported

Optimal time for initiating antiretroviral therapy (ART) in HIV-infected, treatment-naive children aged 2 to 5 years old (Review) 
Ananworanich 2008 (Continued)

\begin{tabular}{|c|c|c|}
\hline Allocation concealment (selection bias) & Unclear risk & Not reported \\
\hline $\begin{array}{l}\text { Blinding of participants and personnel } \\
\text { (performance bias) } \\
\text { All outcomes }\end{array}$ & High risk & $\begin{array}{l}\text { This was an open-label trial so personnel } \\
\text { and caregivers were not blinded, potentially } \\
\text { introducing performance bias }\end{array}$ \\
\hline $\begin{array}{l}\text { Blinding of outcome assessment (detection } \\
\text { bias) } \\
\text { All outcomes }\end{array}$ & Unclear risk & Not reported \\
\hline $\begin{array}{l}\text { Incomplete outcome data (attrition bias) } \\
\text { All outcomes }\end{array}$ & Unclear risk & $\begin{array}{l}\text { In the IMMEDIATE group, attrition was } \\
1 / 24(4.2 \%) \text { and in the DEFERRED } \\
\text { group, attrition was } 2 / 19(10.5 \%) \text {. We } \\
\text { judged this to be low risk }\end{array}$ \\
\hline Selective reporting (reporting bias) & Low risk & $\begin{array}{l}\text { We were not able to identify a registered } \\
\text { protocol for the trial to compare registered } \\
\text { and reported outcomes. However, given } \\
\text { that the trial was designed as a feasibility } \\
\text { study with recruitment rate, adherence and } \\
\text { retention as primary outcomes rather than } \\
\text { measures of efficacy, we judged the risk of } \\
\text { selective reporting to be low }\end{array}$ \\
\hline $\begin{array}{l}\text { Control of time-dependent confounding } \\
\text { COHORT ONLY }\end{array}$ & Low risk & $\begin{array}{l}\text { Not applicable due to the nature of ran- } \\
\text { domisation which eliminates the need to } \\
\text { control for confounding }\end{array}$ \\
\hline Other bias & Unclear risk & $\begin{array}{l}\text { The trial was conducted to ascertain the } \\
\text { feasibility of the larger PREDICT } 2012 \\
\text { trial and was intentionally not powered to } \\
\text { evaluate efficacy. We did not identify other } \\
\text { sources of bias }\end{array}$ \\
\hline
\end{tabular}


Methods

Participants
STUDY TYPE:

- Randomised controlled trial

COUNTRY:

- Thailand

- Cambodia

SETTING:

- Nine tertiary referral hospitals and research sites of the Comprehensive

International Program for Research in IADS (CIPRA) - Thailand and Cambodia Network

DURATION OF RECRUITMENT:

- Mar 2006 - Sep 2008

DURATION OF TRIAL:

- 5 years and 3 months. Trial completed in May 2011

FOLLOW-UP:

- Length of follow-up was 144 weeks.

- Children in the IMMEDIATE group were followed at weeks 2, 4, 8, 12 and then every 12 weeks thereafter; children in the DEFERRED group were followed at weeks 8,12 and every 12 weeks thereafter

- At baseline, all children were evaluated clinically, and complete blood count, CD4\% and count, serum electrolytes and alanine aminotransferase (ALT) were performed

- At every follow-up visit, clinical evaluation was done including an assessment for toxicity or HIV-related events

- At every 12 week visit, complete blood count, CD4\% and count, serum electrolytes and Alanine Transferase (ALT) were performed

- At every 24 week visit, plasma HIV RNA (viral load) and Beery Visual Motor Integration (VMI) tests were conducted

- $96 \%$ of children completed 144 weeks of follow-up.

\section{INCLUSION CRITERIA:}

- Children aged one to 12 years old, with HIV infection (defined as positive HIV DNA PCR or RNA PCR twice among children between 12 and 18 months, or with positive HIV antibody test among children aged $>18$ months)

- CD $4 \% 15$ - 24\%

- No history of AIDS illness (CDC C events)

- Never received ART other than for prevention of MTCT

EXCLUSION CRITERIA:

- Children younger than one year

- Active AIDS-defining illness

- Use of immunosuppressive drugs

- Use of immuno-modulators within 30 days prior to study entry

- Abnormal laboratory results:

- Absolute neutrophil count $<750$ cells $/ \mathrm{mm}^{3}$

○ Haemoglobin $<7.5 \mathrm{~g} / \mathrm{dL}$

- Platelet count $<50,000 / \mathrm{mm}^{3}$

- ALT > 4 times upper limit of normal (ULN)

Number of participants randomised: 300

Baseline data:

- Median age at randomization (IQR): IMMEDIATE group: 6.4 (IQR: 3.6 - 8.0) 
- Number and \% in age groups ( $\mathrm{n}(\%))$ :

○ 1 - 3 years: IMMEDIATE group: 45 (30); DEFERRED group: 33 (22)

○ 4 - 6 years: IMMEDIATE group: 43 (29); DEFERRED group: 50 (33)

○ 7 - 9 years: IMMEDIATE group: 44 (30); DEFERRED group: 49 (33)

- 10 - 12 years: IMMEDIATE group: 17 (11); DEFERRED group: 18 (12)

- Gender distribution (Male: Female) (n, \%): IMMEDIATE group:77: 72 (48; 52); DEFERRED group: 54:96 (36: 64)

- Median weight for age z-scores (WAZ) (IQR): IMMEDIATE group: -1.3 (-2.0 to -0.8); DEFERRED group: -1.3 (-2.0 to -0.8$)$

- Median height for age z-scores (WAZ) (IQR): IMMEDIATE group: -1.6 (-2.5 to -0.8); DEFERRED group: -1.7 (-2.6 to -0.9$)$

- Median percent CD4 count (\%; IQR): IMMEDIATE group: 19 (16 - 22);

DEFERRED group: 20 (17 - 23)

- Median CD4 count (cells/mm³ $\mathrm{mm}^{3}$ IQR): IMMEDIATE group: 620 (425 - 851); DEFERRED group: 619 (466 - 847)

Baseline characteristics were similar between the two groups except for gender. There was a significantly greater proportion of females in the deferred arm ( $\mathrm{p}$ value not reported)

Interventions

Optimal time for initiating antiretroviral therapy (ART) in HIV-infected, treatment-naive children aged 2 to 5 years old (Review)

Copyright $\Subset 2013$ The Cochrane Collaboration. Published by John Wiley \& Sons, Ltd.
INTERVENTION: IMMEDIATE GROUP

- Participants were started on ART immediately at study entry CONTROL: DEFERRED GROUP

- Participants were started on ART during the trial if

- Development of CDC category C events OR children OR

- Confirmed CD4\% decline to $<15 \%$ prior to December 2008 for all

- Confirmed CD $4 \%$ decline to $<20 \%$ in children aged 1 to 3 years from December 2008*

*The change in the immunologic criteria was due to a change in World Health Organization and national treatment guidelines. Recruitment was completed in September 2008 prior to implementing the change

First-line ART comprised:

- Zidovudine, lamivudine and nevirapine

- A protease inhibitor (lopinavir/ritonavir or nelfinavir) was substituted for nevirapine in children with prior exposure to nevirapine (nelfinavir was not used after September 2007)

- Abacavir was substituted for zidovudine in cases of grade 3 or 4 hematologic toxicity

- Efavirenz or a protease inhibitor was substituted for nevirapine in children with nevirapine- hypersensitivity depending on the severity

- Children also requiring anti-tuberculosis treatment received zidovudine, lamivudine and abacavir

Second-line ART:

- ART treatment failure was defined as HIV RNA $>1000$ copies $/ \mathrm{ml}$ after $\geq 6$ months of treatment

- ART selection was based on genotypic resistance testing. COMPLIANCE:

Adherence questionnaires and pill counts were used to assess adherence. Good adherence (defined as average adherence by pill count of $>95 \%$ while receiving study drug) was 
PREDICT 2012 (Continued)

reported in $88 \%$ of the IMMEDIATE and $90 \%$ of the DEFERRED group

CO-INTERVENTIONS:

Cotrimoxazole was started immediately with the first decrease of CD4 below 15\% and was continued for at least six months until two consecutive CD4 were above $15 \%$

Outcomes

PRIMARY OUTCOMES:

- CDC Category C event-free (AIDS-fee) survival at week 144

SECONDARY OUTCOMES:

- CDC Category B events

- Beery VM standard score

- Hospitalization rates

- CD4\% changes

- Growth changes

- Cumulative proportion of children with virologic failure

- ART-related Adverse Events (measured using the 2004 Adult and Pediatric

Grading Tables of the Division of AIDS, NIH.

$\begin{array}{ll}\text { Notes } & \text { ETHICS } \\ & \text { Institutional Review Board permission obtained at all sites. } \\ & \text { INFORMED CONSENT: } \\ \text { All caregivers gave written informed consent. } & \text { FUNDING } \\ & \text { Division of AIDS (DAIDS), National Institute of Allergy and Infectious Diseases, Na- } \\ & \text { tional Institute of Child Health and Human Development (NICHD) and National In- } \\ & \text { stitute of Mental Health (NIMH), US National Institutes of Health (NIH) } \\ & \text { Antiretroviral drugs were provided by ViiV Healthcare/GlaxoSmithKline (zidovudine, } \\ \text { lamivudine and abacavir), Boehringer-Ingelheim (nevirapine), Merck (efavirenz), Abbott } \\ \text { (lopinavir/ritonavir) and Roche (nelfinavir) }\end{array}$

Risk of bias

Bias

Random sequence generation (selection Low risk bias)

\section{Support for judgement}

Computer-generated randomisation program using SAS 9.1. The randomisation employed minimization by research site and history of nevirapine exposure

The process was done centrally at a trial coordinating centre in Bangkok and assignment was communicated to the site investigator via fax

Caregivers and personnel were not blinded as the study was open-label. This may introduce performance bias (performance bias)

All outcomes 
PREDICT 2012

Blinding of outcome assessment (detection Low risk bias)

All outcomes
An independent committee blinded to assignment, CD4 and ART status, reviewed outcomes of CDC category B and C endpoints and hospitalizations. Other outcomes may have been susceptible to detection bias but we judged this to be of low risk

In the IMMEDIATE group, 7/150 (4. $6 \%)$ were lost-to-follow-up and in the DEFERRED group, 3/150 (2\%) were lost-tofollow-up. This represents a low attrition rate

We compared the trial report with the entry for NCT00234091 on www.clinicaltrials. gov. There was no selective reporting

Not applicable due to the nature of randomisation which eliminates the need to control for confounding

Control of time-dependent confounding Low risk COHORT ONLY

Low risk

Low risk
The trial was funded by government organizations. The drugs were supplied by pharmaceutical companies which had no role in the study design, analysis or manuscript preparation. The trial was not stopped early. For these reasons we judged the risk of bias to be low for other forms of bias 
- Observational cohort study using prospectively routinely collected data COUNTRY:

- South Africa

SETTING:

- Harriet Shezi Children's Clinic, an outpatient paediatric clinic at Chris Hani Baragwanath Hospital in Soweto (tertiary facility)

DURATION OF RECRUITMENT:

- Apr 2004 - Mar 2008

DURATION OF TRIAL:

- 4 years. Completed on 31 March 2008 or at last visit before 31 March 2008 by administrative censoring FOLLOW-UP:

- Median length of follow-up 9.6 months (IQR: 1.9 - 23.1 months)

- Children were followed after one month, then at three months and then every three months or as clinically indicated

- At baseline, laboratory investigations (CD4 cell count and viral load) were conducted

- At every 6 monthly visit, CD4 and viral load were conducted on when indicated

- Children (age defined as younger than 15 years old and assumed to be excluding infants (less than one years old)), with HIV infection, with tuberculosis (TB) infection diagnosed by clinical grounds including:

- Failure to thrive

- Prolonged (more than 2 weeks) cough

- Suspicious chest radiograph

- With or without positive contact history

- Bacteriological confirmation was attempted in older children who could produce sputum samples

- TB treatment must have been initiated prior to ART initiation EXCLUSION CRITERIA:

- Children already on TB treatment at first visit in the clinic

Number of participants eligible for inclusion: 573

- Median age of all children at baseline (age in years; IQR): 3.5 years (1.4 - 6.8)

- Gender distribution (Male: Female) (n, \%): Not reported

- Median weight for age z-scores of all children at baseline (WAZ) (IQR): -2.3 (-3.6 to -1.3 )

- Median height for age z-scores of all children at baseline (WAZ) (IQR): Not reported

- Median percent CD4 count of all children at baseline (\%; IQR): 11.9\% (6.6 - 18. 3)

- Median viral load for all children at baseline (log copies/ml; IQR): 5.2 (4.5 - 5.9) Characteristics and baseline data were compared between those who initiated ART and those who did not initiate ART and between those who initiated ART within one month from enrolment and those who initiated ART equal to or greater than one month from enrolment. As the comparison this review is focused on is timing on ART initiation, the baseline results are presented for those who initiated ART within one month $(\mathrm{n}=288)$ and greater or equal to one month $(n=206)$ below: 
Yotebieng 2010 (Continued)

- Median age of children at TB treatment initiation (age in years; IQR): ART INITIATED < 1 MONTH: 3.5 years $(1.4$ - 7.1); ART INITIATED $\geq 1$ MONTH: 3 . $5(1.4-6.7)$

- Median weight for age z-scores of children: (WAZ) (IQR): ART INITIATED < 1 MONTH: -2.71 (-4.11 to 1.60$)$; ART INITIATED $\geq 1$ MONTH: -1.92 (-2.92 to -0 . 83)

- Median percent CD4 count of children (\%; IQR): ART INITIATED < 1 MONTH 8.0 (4.6 - 13.6): ART INITIATED $\geq 1$ MONTH: 15.0 (9.8-21.2)

- Median CD4 cell count of children (count/microL; IQR): ART INITIATED < 1 MONTH 273 (98 - 604): ART INITIATED $\geq 1$ MONTH: 533 (238 - 868)

- Median viral load for children (log copies/ml; IQR): ART INITIATED $<1$ MONTH: 5.3 (4.6 - 6.0); ART INITIATED $\geq 1$ MONTH: 5.2 (4.5 - 5.8)

- Median time from TB to ART initiation (days; IQR): ART INITIATED $<1$ MONTH: 4 (0 - 14); ART INITIATED $\geq 1$ MONTH: 59 (42 - 130)

The baseline difference between the groups was statistically significant for CD4 cell count, CD4 cell percentage, WAZ, and time from TB to ART initiation

The authors report that the distribution of baseline characteristics was similar to that of the above for 15 and 60 day cut-offs. The actual data is not reported

\section{Interventions}

\section{INTERVENTION: ART INITIATED $<1$ MONTH}

ART was initiated in children within one month of enrolment CONTROL: ART INITIATED $\geq 1 \mathrm{MONTH}$

ART was initiated in children after one month or more of enrolment The authors also consider the data in 15-day and 60-day cut-offs

First-line ART regimen at the time, according to South African National Guidlines. comprised stavudine, lamivudine, and ritonavir-boosted lopinavir for children three years or younger; or stavudine, lamivudine and efavirenz for those over three years and over $10 \mathrm{~kg}$ of weight. Double doses of ritonavir were given during anti-TB treatment CO-INTERVENTIONS:

All children were receiving TB treatment which comprised a combination of rifampicin, isoniazid, and pyrazinamide for the initial two months followed by rifampicin and isoniazid for the remaining four months

- Survival (time from TB treatment initiation to death)

- Time to viral suppression (time from ART initiation to date of first viral load measure below 400 HIV RNA copies/ml)

Not reported. Routine data collection INFORMED CONSENT:

Not reported.

FUNDING

US National Institutes of Health (NIH) Fogarty grant: DHHS/NIH/FIC 5 D43 TW01039-08 AIDS International Training and Research Program at the University of North Carolina (UNC) at Chapel Hill. Additional support from the UNC Center of Global Initiative and the American International Health Alliance

Antiretroviral drugs were provided by ViiV Healthcare/GlaxoSmithKline (zidovudine, lamivudine and abacavir), Boehringer-Ingelheim (nevirapine), Merck (efavirenz), Abbott 
Yotebieng 2010 (Continued)

(lopinavir/ritonavir) and Roche (nelfinavir)

\section{Risk of bias}

\begin{tabular}{|c|c|c|}
\hline Bias & Authors' judgement & Support for judgement \\
\hline $\begin{array}{l}\text { Random sequence generation (selection } \\
\text { bias) }\end{array}$ & High risk & $\begin{array}{l}\text { The groups were not randomised as this } \\
\text { was a cohort study. }\end{array}$ \\
\hline
\end{tabular}

Allocation concealment (selection bias) High risk

The groups were not randomised as this was a cohort study.

Blinding of participants and personnel High risk (performance bias)

As the personnel determined when to iniAll outcomes tiate ART, blinding was not possible and performance bias may be present

Blinding of outcome assessment (detection Low risk bias)

All outcomes

The authors do not report if the assessment was blinded. However as the outcomes are death and viral load lack of blinding is unlikely to be a major source of bias

Incomplete outcome data (attrition bias) Low risk All outcomes

The overall loss to follow-up was 13\% (75/ 573) overall with 38 lost prior to ART initiation and 37 while on ART. The authors report that those children lost to follow-up did not differ to those in care in any of the baseline characteristics or timing of ART initiation

Selective reporting (reporting bias) Unclear risk

No protocol was obtained for this study and there is no report of ethical clearance. As it is based on analysis of routinely collected data there is a risk of selective reporting of those outcomes which were found to be significant or noteworthy in preference over other outcomes. However, given that the outcomes of death and viral suppression are of primary interest to the research question, we did not rate the risk as high but as unclear

Control of time-dependent confounding Low risk COHORT ONLY

The authors made use of inverse probability-of-treatment and censoring (IPTC) weighting of marginal structural models, an appropriate statistical analysis to control for time-dependent confounding

Optimal time for initiating antiretroviral therapy (ART) in HIV-infected, treatment-naive children aged 2 to 5 years old (Review) 
Yotebieng 2010 (Continued)

\begin{tabular}{|c|c|c|}
\hline Other bias & Low risk & $\begin{array}{l}\text { The authors state that the funding sources } \\
\text { had no role in the design and conduct of } \\
\text { this study }\end{array}$ \\
\hline
\end{tabular}

\section{Characteristics of excluded studies [ordered by study ID]}

\begin{tabular}{ll}
\hline Study & Reason for exclusion \\
\hline Edmonds 2009 & $\begin{array}{l}\text { The analysis estimated the effect of ART versus no ART on the incidence of TB (rather than the effect of starting } \\
\text { ART earlier or later) }\end{array}$ \\
\hline Edmonds 2011 & $\begin{array}{l}\text { The analysis estimated the effect of ART versus no ART on the incidence of TB (rather than the effect of starting } \\
\text { ART earlier or later) }\end{array}$ \\
\hline Munyagwa 2012 & $\begin{array}{l}\text { This analysis compared ART versus no ART and was not appropriately adjusted for (it did not consider time- } \\
\text { dependent confounding) }\end{array}$ \\
\hline Musoke 2010 & $\begin{array}{l}\text { This study analyzed growth trajectories and immunological and virological response of children starting ART. } \\
\text { There were no morbidity/mortality endpoints and no appropriate comparisons of starting early versus starting } \\
\text { late }\end{array}$ \\
\hline Patel 2008 a & $\begin{array}{l}\text { The analysis did not include mortality and morbidity data and estimated the effect of ART versus no ART (rather } \\
\text { than the effect of starting earlier or later.) }\end{array}$ \\
\hline Patel 2008 b & $\begin{array}{l}\text { In this study the analysis estimated the effect of ART versus no ART (rather than the effect of starting ART earlier } \\
\text { or later) }\end{array}$ \\
\hline
\end{tabular}


DATA ANDANALYSES

Comparison 1. IMMEDIATE versus DEFERRED initiation of ART all ages (RCT)

\begin{tabular}{|c|c|c|c|c|}
\hline Outcome or subgroup title & $\begin{array}{l}\text { No. of } \\
\text { studies }\end{array}$ & $\begin{array}{c}\text { No. of } \\
\text { participants }\end{array}$ & Statistical method & Effect size \\
\hline 1 Death & 2 & 343 & Risk Ratio (M-H, Random, 95\% CI) & $3.0[0.12,73.06]$ \\
\hline $\begin{array}{l}2 \text { CDC Category C disease } \\
\text { (number of children) }\end{array}$ & 2 & 343 & Risk Ratio (M-H, Random, 95\% CI) & $1.5[0.25,8.85]$ \\
\hline $\begin{array}{l}3 \text { CDC Category B disease } \\
\text { (numbers of children) Relative } \\
\text { Risk }\end{array}$ & 2 & 343 & Risk Ratio (M-H, Random, 95\% CI) & $1.42[0.14,14.28]$ \\
\hline $\begin{array}{l}4 \text { CDC Category B disease } \\
\text { (numbers of children) Peto } \\
\text { Odds Ratio }\end{array}$ & 2 & 343 & Peto Odds Ratio (Peto, Fixed, 95\% CI) & $0.70[0.39,1.24]$ \\
\hline $\begin{array}{l}5 \text { Pulmonary TB (clinically } \\
\text { diagnosed) }\end{array}$ & 2 & 343 & Risk Ratio (M-H, Fixed, 95\% CI) & $3.21[0.52,19.89]$ \\
\hline $\begin{array}{l}6 \text { Median time before development } \\
\text { of CDC B or C event }\end{array}$ & & & Other data & No numeric data \\
\hline $\begin{array}{l}7 \text { Proportion of children on ART } \\
\text { with HIV-RNA }<50 \text { copies } / \mathrm{ml}\end{array}$ & 2 & 238 & Risk Ratio (M-H, Random, 95\% CI) & $0.96[0.84,1.09]$ \\
\hline 8 Median CD $4 \%$ at study end & & & Other data & No numeric data \\
\hline 9 Mean CD $4 \%$ at week 144 & 1 & 300 & Mean Difference (IV, Random, 95\% CI) & $8.40[6.83,9.97]$ \\
\hline $\begin{array}{l}10 \text { Proportion of children with } \\
\text { CD } 4 \%<15 \% \text { at study end }\end{array}$ & 2 & 343 & Risk Ratio (M-H, Random, 95\% CI) & $0.11[0.02,0.60]$ \\
\hline $\begin{array}{l}11 \text { Mean weight gain per year in } \\
\mathrm{kg}\end{array}$ & 1 & 299 & Mean Difference (IV, Fixed, 95\% CI) & $0.10[-0.16,0.36]$ \\
\hline $\begin{array}{l}12 \text { Median weight-for-age } \mathrm{Z} \text { score } \\
\text { at study end ( } 134 \text { - } 144 \text { weeks) }\end{array}$ & & & Other data & No numeric data \\
\hline $\begin{array}{l}13 \text { Mean height gain per year in } \\
\mathrm{cm}\end{array}$ & 1 & 300 & Mean Difference (IV, Fixed, 95\% CI) & $0.5[0.20,0.80]$ \\
\hline $\begin{array}{l}14 \text { Median height-for-age } \mathrm{Z} \text { score } \\
\text { at study end ( } 134 \text { - } 144 \text { weeks) }\end{array}$ & & & Other data & No numeric data \\
\hline $\begin{array}{l}15 \text { Mean standardized score on } \\
\text { Beery VMI at } 144 \text { weeks }\end{array}$ & 1 & 272 & Mean Difference (IV, Fixed, 95\% CI) & $-1.40[-4.70,1.90]$ \\
\hline $\begin{array}{l}16 \text { Proportion of children with } \\
\text { adverse events }\end{array}$ & 1 & 43 & Risk Ratio (M-H, Random, 95\% CI) & $0.92[0.80,1.07]$ \\
\hline $\begin{array}{l}17 \text { Proportion of children with } \\
\text { ART-related adverse events }\end{array}$ & 2 & 343 & Risk Ratio (M-H, Random, 95\% CI) & $1.87[0.77,4.51]$ \\
\hline
\end{tabular}

Optimal time for initiating antiretroviral therapy (ART) in HIV-infected, treatment-naive children aged 2 to 5 years old (Review) 
Comparison 2. SUBGROUP ANALYSIS: IMMEDIATE versus DEFERRED initiation of ART 24 to 59 months (RCT)

\begin{tabular}{|c|c|c|c|c|}
\hline Outcome or subgroup title & $\begin{array}{l}\text { No. of } \\
\text { studies }\end{array}$ & $\begin{array}{c}\text { No. of } \\
\text { participants }\end{array}$ & Statistical method & Effect size \\
\hline 1 Death & 2 & 122 & Risk Ratio (M-H, Random, 95\% CI) & $2.88[0.12,68.88]$ \\
\hline $\begin{array}{l}2 \text { CDC Category C disease } \\
\text { (number of children) }\end{array}$ & 2 & 122 & Risk Ratio (M-H, Random, 95\% CI) & $0.96[0.06,14.87]$ \\
\hline $\begin{array}{l}3 \text { CDC Category B disease } \\
\text { (numbers of children) Relative } \\
\text { Risk }\end{array}$ & 2 & 122 & Risk Ratio (M-H, Random, 95\% CI) & $0.95[0.24,3.73]$ \\
\hline $\begin{array}{l}4 \text { CDC Category B disease } \\
\text { (numbers of children) Peto } \\
\text { Odds Ratio }\end{array}$ & 2 & 122 & Peto Odds Ratio (Peto, Fixed, 95\% CI) & $0.76[0.29,2.02]$ \\
\hline $\begin{array}{l}5 \text { Pulmonary TB (clinically } \\
\text { diagnosed) }\end{array}$ & 2 & 122 & Risk Ratio (M-H, Fixed, 95\% CI) & $1.19[0.19,7.27]$ \\
\hline $\begin{array}{l}6 \text { Proportion of children on ART } \\
\text { with HIV-RNA }<50 \text { copies } / \mathrm{ml}\end{array}$ & 1 & 67 & Risk Ratio (M-H, Random, 95\% CI) & $1.11[0.86,1.43]$ \\
\hline 7 Mean CD $4 \%$ at week 144 & 1 & 94 & Mean Difference (IV, Random, 95\% CI) & $5.90[2.74,9.06]$ \\
\hline $\begin{array}{l}8 \text { Proportion of children with } \\
\text { CD } 4 \%<15 \% \text { at study end }\end{array}$ & 2 & 122 & Risk Ratio (M-H, Random, 95\% CI) & $0.40[0.06,2.52]$ \\
\hline 9 Mean weight gain per year in $\mathrm{kg}$ & 1 & 94 & Mean Difference (IV, Fixed, 95\% CI) & $-0.03[-0.25,0.19]$ \\
\hline $\begin{array}{l}10 \text { Mean height gain per year in } \\
\mathrm{cm}\end{array}$ & 1 & 94 & Mean Difference (IV, Fixed, 95\% CI) & $0.30[-0.28,0.88]$ \\
\hline $\begin{array}{l}11 \text { Mean standardized score on } \\
\text { Beery VMI at } 144 \text { weeks }\end{array}$ & 1 & 82 & Mean Difference (IV, Fixed, 95\% CI) & $2.30[-4.37,8.97]$ \\
\hline $\begin{array}{l}12 \text { Proportion of children with } \\
\text { ART-related Grade } 3 \text { or } 4 \\
\text { adverse events }\end{array}$ & 1 & 94 & Risk Ratio (M-H, Random, 95\% CI) & $0.48[0.04,5.11]$ \\
\hline
\end{tabular}

Comparison 3. ADJUSTED/WEIGHTED EARLY vs DEFERRED initiation of ART in children with TB and HIV

\begin{tabular}{|c|c|c|c|c|}
\hline Outcome or subgroup title & $\begin{array}{l}\text { No. of } \\
\text { studies }\end{array}$ & $\begin{array}{c}\text { No. of } \\
\text { participants }\end{array}$ & Statistical method & Effect size \\
\hline 1 Death & 1 & & Hazard Ratio (Fixed, 95\% CI) & Subtotals only \\
\hline $\begin{array}{l}1.1>15 \text { DAYS vs }=<15 \\
\text { DAYS }\end{array}$ & 1 & 501 & Hazard Ratio (Fixed, 95\% CI) & $0.82[0.52,1.31]$ \\
\hline $\begin{array}{l}1.2>30 \text { DAYS vs }=<30 \\
\text { DAYS }\end{array}$ & 1 & 494 & Hazard Ratio (Fixed, 95\% CI) & $0.86[0.49,1.52]$ \\
\hline $\begin{array}{l}1.3>60 \text { DAYS vs }=<60 \\
\text { DAYS }\end{array}$ & 1 & 489 & Hazard Ratio (Fixed, 95\% CI) & $1.32[0.36,4.87]$ \\
\hline 2 Virologic suppression & 1 & & Hazard Ratio (Fixed, 95\% CI) & Subtotals only \\
\hline $\begin{array}{l}2.1>15 \text { DAYS vs }=<15 \\
\text { DAYS }\end{array}$ & 1 & 324 & Hazard Ratio (Fixed, 95\% CI) & $0.98[0.76,1.26]$ \\
\hline
\end{tabular}

Optimal time for initiating antiretroviral therapy (ART) in HIV-infected, treatment-naive children aged 2 to 5 years old (Review) 


\begin{tabular}{lllll}
$2.2>30$ DAYS vs $=<30$ & 1 & 324 & Hazard Ratio (Fixed, 95\% CI) & $0.95[0.74,1.22]$ \\
DAYS & & & & $0.84[0.64,1.10]$ \\
$2.3>60$ DAYS vs $=<60$ & 1 & 324 & Hazard Ratio (Fixed, 95\% CI) \\
DAYS & & & & \\
\hline
\end{tabular}

\section{Analysis I.I. Comparison I IMMEDIATE versus DEFERRED initiation of ART all ages (RCT), Outcome I} Death.

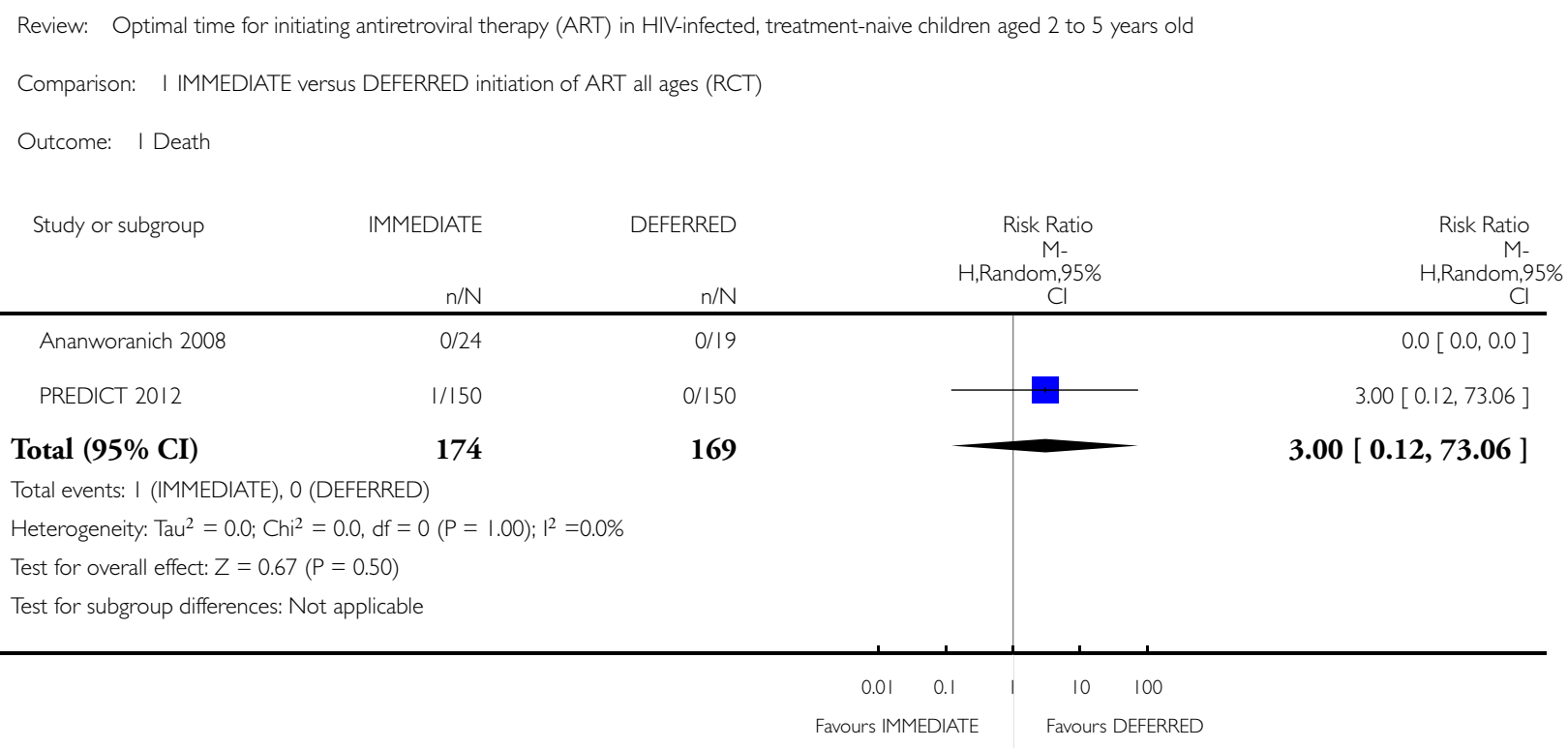


Analysis I.2. Comparison I IMMEDIATE versus DEFERRED initiation of ART all ages (RCT), Outcome 2 CDC Category $C$ disease (number of children).

Review: Optimal time for initiating antiretroviral therapy (ART) in HIV-infected, treatment-naive children aged 2 to 5 years old

Comparison: I IMMEDIATE versus DEFERRED initiation of ART all ages (RCT)

Outcome: 2 CDC Category C disease (number of children)

Study or subgroup $\quad$ IMMEDIATE $\quad$ DEFERRED Risk Ratio

H,Random,95\% H,Random,95\%

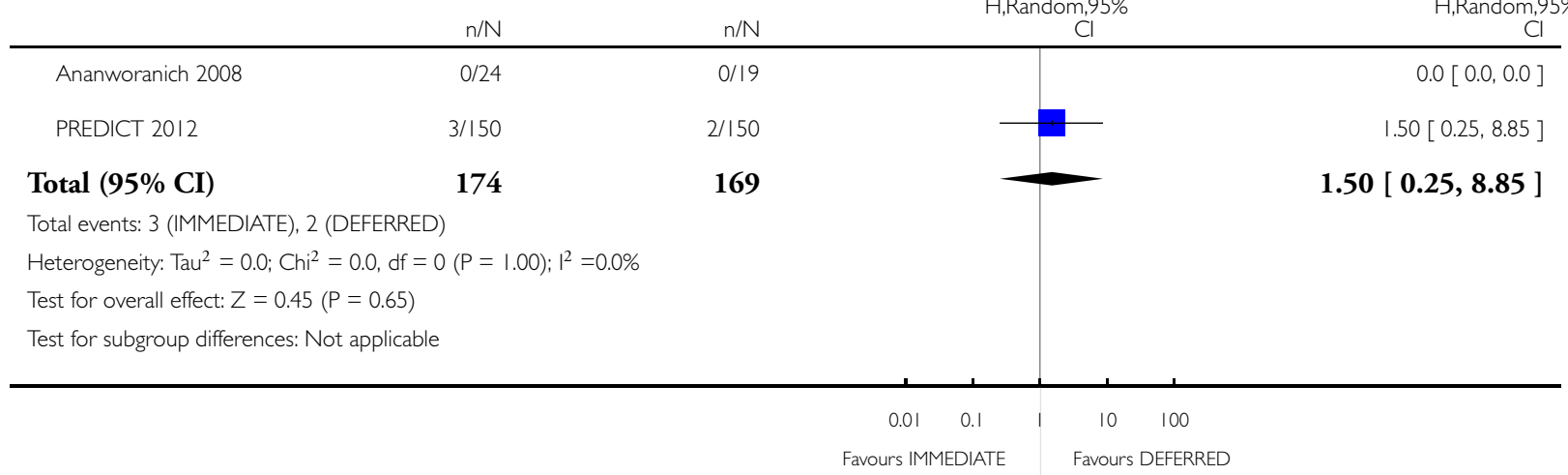

Optimal time for initiating antiretroviral therapy (ART) in HIV-infected, treatment-naive children aged 2 to 5 years old (Review) 
Analysis I.3. Comparison I IMMEDIATE versus DEFERRED initiation of ART all ages (RCT), Outcome 3 CDC Category B disease (numbers of children) Relative Risk.

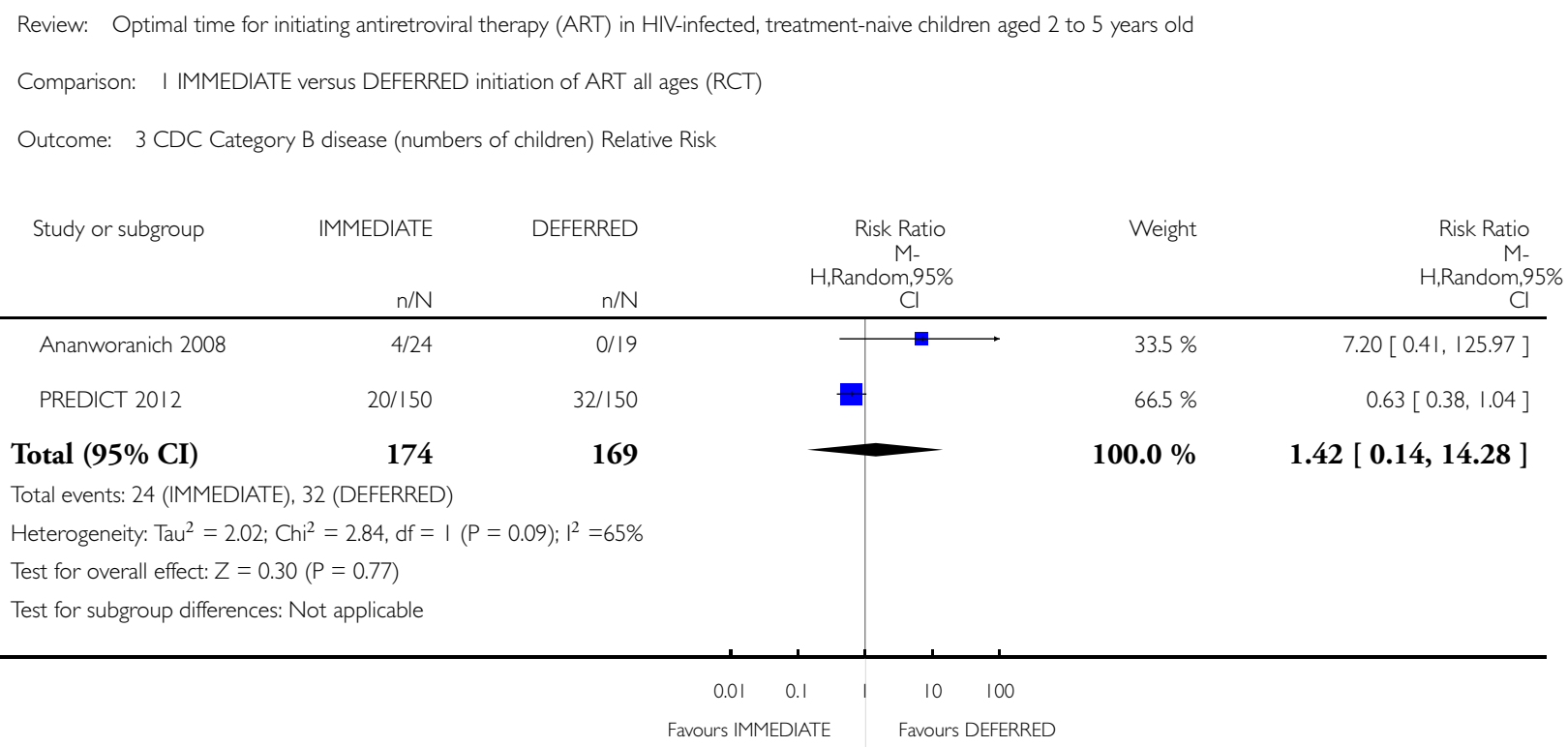




\section{Analysis I.4. Comparison I IMMEDIATE versus DEFERRED initiation of ART all ages (RCT), Outcome 4} CDC Category B disease (numbers of children) Peto Odds Ratio.

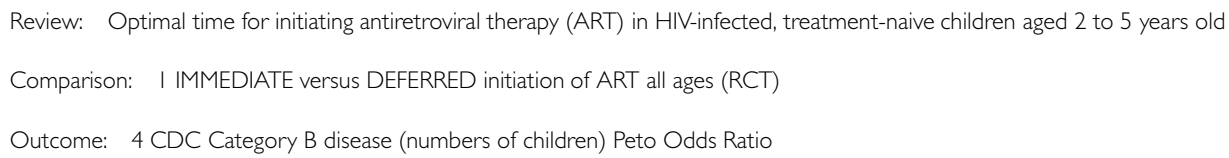

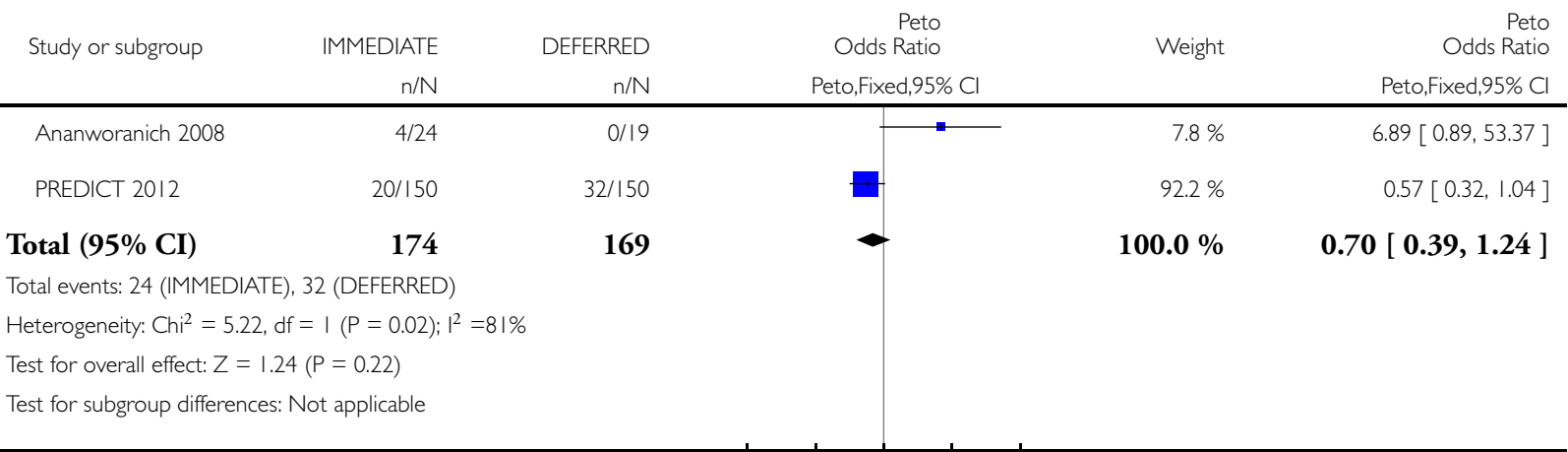

Analysis I.5. Comparison I IMMEDIATE versus DEFERRED initiation of ART all ages (RCT), Outcome 5 Pulmonary TB (clinically diagnosed).

Review: Optimal time for initiating antiretroviral therapy (ART) in HIV-infected, treatment-naive children aged 2 to 5 years old

Comparison: I IMMEDIATE versus DEFERRED initiation of ART all ages (RCT)

Outcome: 5 Pulmonary TB (clinically diagnosed)

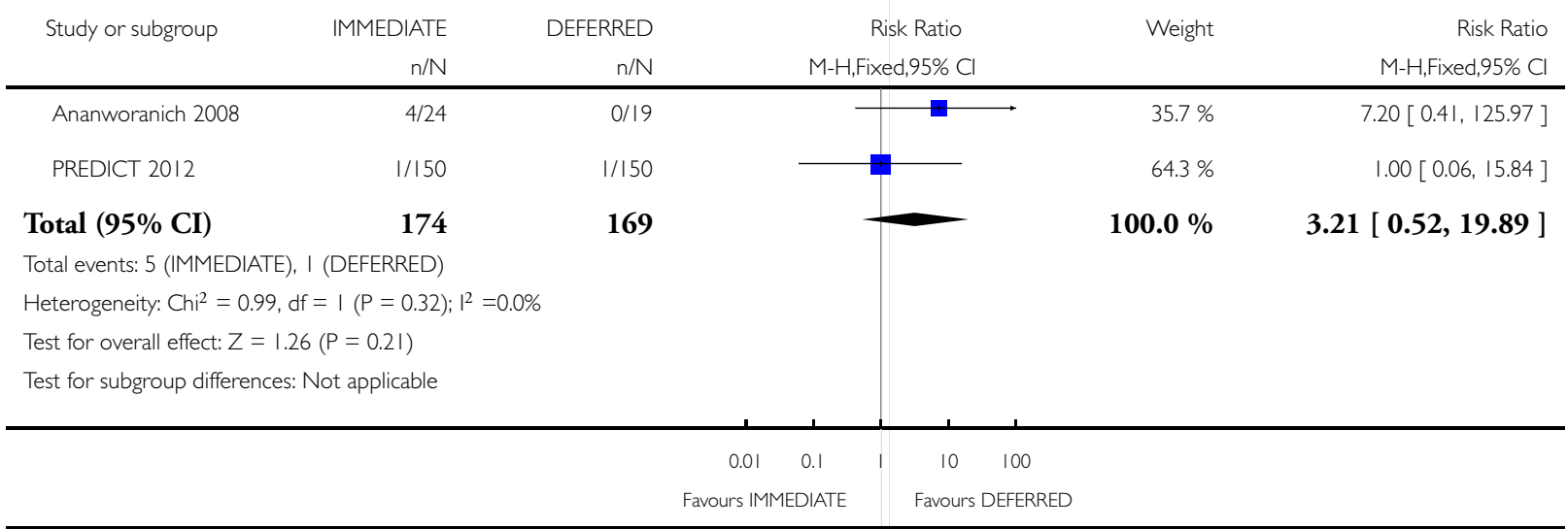


Analysis I.6. Comparison I IMMEDIATE versus DEFERRED initiation of ART all ages (RCT), Outcome 6 Median time before development of CDC B or C event.

Median time before development of CDC B or C event

\begin{tabular}{l|l|l}
\hline Study & IMMEDIATE (time on ART before events) & DEFERRED (time from enrolment to events) \\
\hline Ananworanich 2008 & 60 (IQR: $48-72)$ weeks & Nil \\
\hline PREDICT 2012 & 9 (IQR: $4-30)$ weeks & 57 (IQR: $34-101)$ weeks \\
\hline
\end{tabular}

Analysis I.7. Comparison I IMMEDIATE versus DEFERRED initiation of ART all ages (RCT), Outcome 7 Proportion of children on ART with HIV-RNA $<50$ copies $/ \mathrm{ml}$.

Review: Optimal time for initiating antiretroviral therapy (ART) in HIV-infected, treatment-naive children aged 2 to 5 years old

Comparison: I IMMEDIATE versus DEFERRED initiation of ART all ages (RCT)

Outcome: 7 Proportion of children on ART with HIV-RNA $<50$ copies $/ \mathrm{ml}$

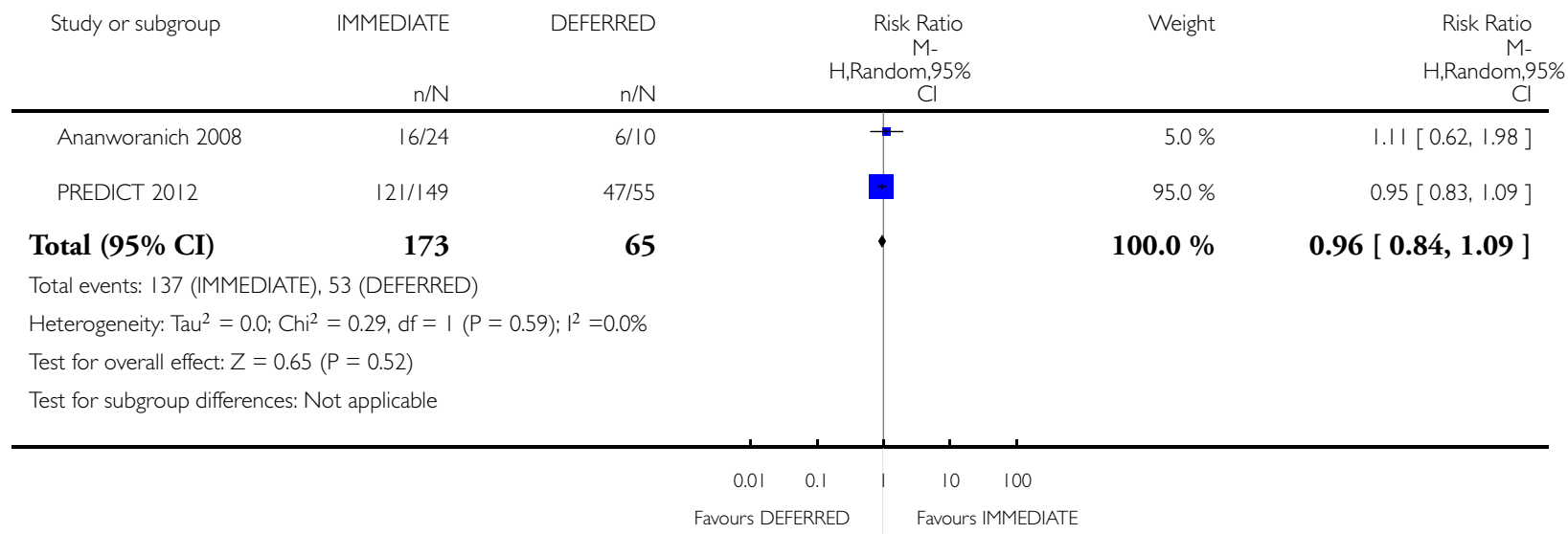

Analysis I.8. Comparison I IMMEDIATE versus DEFERRED initiation of ART all ages (RCT), Outcome 8 Median CD4\% at study end.

Median CD4\% at study end

\begin{tabular}{l|l|l}
\hline Study & IMMEDIATE & DEFERED value
\end{tabular}

Optimal time for initiating antiretroviral therapy (ART) in HIV-infected, treatment-naive children aged 2 to 5 years old (Review) 
Median CD4\% at study end (Continued)

Ananworanich 2008 Median (IQR): $31(24$ - 39) $\quad$ Median (IQR): 23 (17 - 31) 0.032

Analysis I.9. Comparison I IMMEDIATE versus DEFERRED initiation of ART all ages (RCT), Outcome 9 Mean CD4\% at week 144 .

Review: Optimal time for initiating antiretroviral therapy (ART) in HIV-infected, treatment-naive children aged 2 to 5 years old

Comparison: I IMMEDIATE versus DEFERRED initiation of ART all ages (RCT)

Outcome: 9 Mean CD4\% at week 144

\begin{tabular}{|c|c|c|c|c|c|c|c|c|c|}
\hline \multirow[t]{2}{*}{ Study or subgroup } & \multirow{2}{*}{$\begin{array}{r}\text { IMMEDIATE } \\
\text { N }\end{array}$} & \multicolumn{2}{|r|}{ DEFERRED } & \multicolumn{4}{|c|}{$\begin{array}{r}\text { Mean } \\
\text { Difference }\end{array}$} & Weight & \multirow{2}{*}{$\begin{array}{r}\text { Mean } \\
\text { Difference } \\
\text { IV,Random,95\% Cl }\end{array}$} \\
\hline & & Mean(SD) & N & Mean(SD) & & IV, & dom,95\% Cl & & \\
\hline PREDICT 2012 & 150 & $33.2(6.4)$ & 150 & $24.8(7.4)$ & & & & $100.0 \%$ & $8.40[6.83,9.97]$ \\
\hline Total (95\% CI) & 150 & & 150 & & & & 1 & $100.0 \%$ & $8.40[6.83,9.97]$ \\
\hline \multicolumn{10}{|c|}{ Heterogeneity: not applicable } \\
\hline \multicolumn{10}{|c|}{ Test for overall effect: $Z=10.52(P<0.0000 I)$} \\
\hline \multicolumn{10}{|c|}{ Test for subgroup differences: Not applicable } \\
\hline & & & & & -100 & -50 & 50 & 100 & \\
\hline \multicolumn{10}{|c|}{ Favours DEFERRED } \\
\hline
\end{tabular}

Optimal time for initiating antiretroviral therapy (ART) in HIV-infected, treatment-naive children aged 2 to 5 years old (Review) 
Analysis I.10. Comparison I IMMEDIATE versus DEFERRED initiation of ART all ages (RCT), Outcome I0 Proportion of children with CD4\% < I5\% at study end.

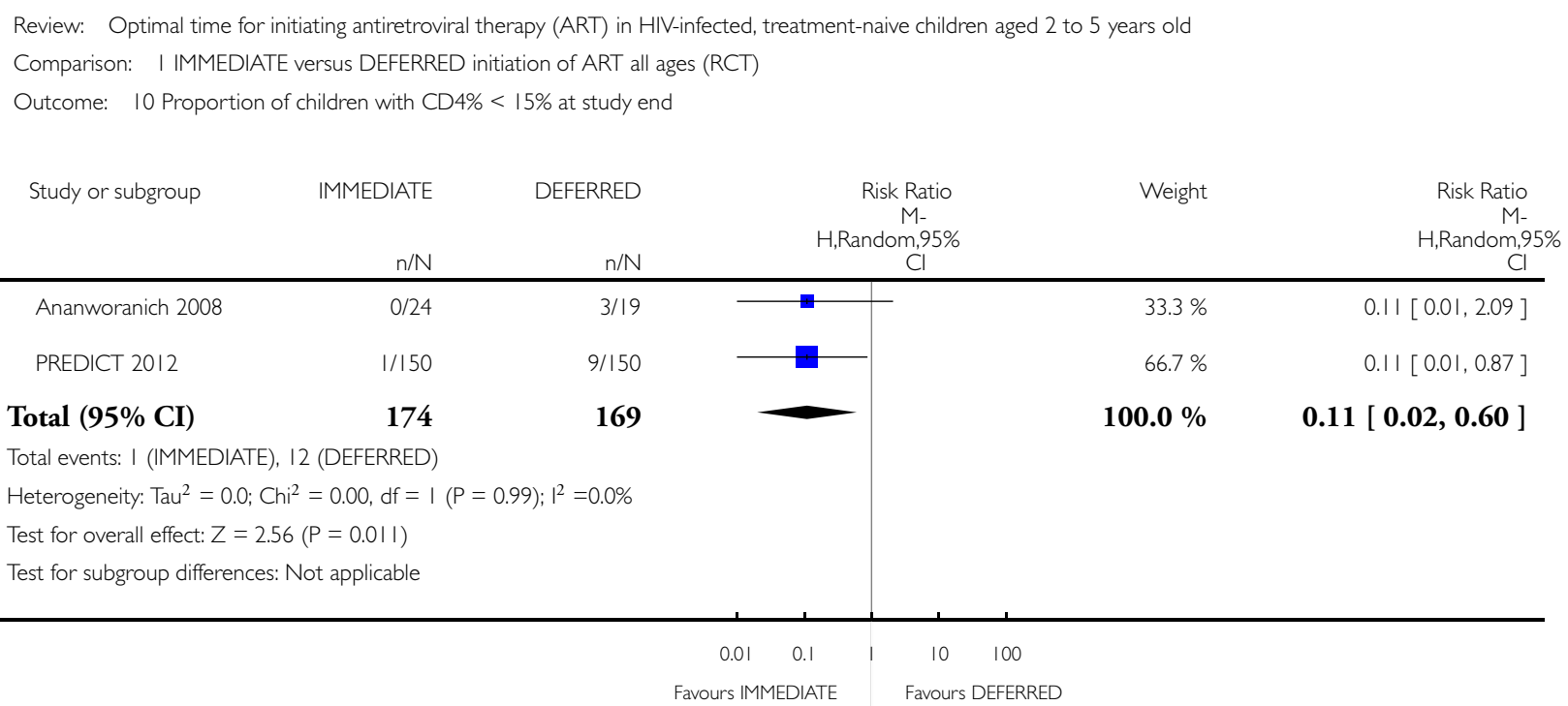

\section{Analysis I.I I. Comparison I IMMEDIATE versus DEFERRED initiation of ART all ages (RCT), Outcome II Mean weight gain per year in $\mathbf{k g}$.}

\footnotetext{
Review: Optimal time for initiating antiretroviral therapy (ART) in HIV-infected, treatment-naive children aged 2 to 5 years old

Comparison: I IMMEDIATE versus DEFERRED initiation of ART all ages (RCT)

Outcome: II Mean weight gain per year in $\mathrm{kg}$
}

\begin{tabular}{|c|c|c|c|c|c|c|c|}
\hline \multirow[t]{2}{*}{ Study or subgroup } & \multirow{2}{*}{$\begin{array}{r}\text { IMMEDIATE } \\
\mathrm{N}\end{array}$} & \multicolumn{3}{|c|}{ DEFERRED } & $\begin{array}{r}\text { Mean } \\
\text { Difference }\end{array}$ & \multirow[t]{2}{*}{ Weight } & \multirow{2}{*}{$\begin{array}{r}\text { Mean } \\
\text { Difference } \\
\text { IV,Fixed,95\% Cl }\end{array}$} \\
\hline & & Mean(SD) & $\mathrm{N}$ & Mean(SD) & IV,Fixed,95\% Cl & & \\
\hline PREDICT 2012 & 149 & $2.2(1.1)$ & 150 & $2.1(1.2)$ & 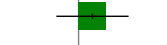 & $100.0 \%$ & $0.10[-0.16,0.36]$ \\
\hline Total $(95 \% \mathrm{CI})$ & 149 & & 150 & & $\longrightarrow$ & $100.0 \%$ & $0.10[-0.16,0.36]$ \\
\hline \multicolumn{8}{|c|}{ Heterogeneity: not applicable } \\
\hline \multicolumn{8}{|c|}{ Test for overall effect: $Z=0.75(P=0.45)$} \\
\hline \multicolumn{8}{|c|}{ Test for subgroup differences: Not applicable } \\
\hline & & & & & 0.5 & 1 & \\
\hline
\end{tabular}


Analysis I.12. Comparison I IMMEDIATE versus DEFERRED initiation of ART all ages (RCT), Outcome I2 Median weight-for-age $Z$ score at study end (I34 - I44 weeks).

Median weight-for-age $Z$ score at study end (134 - 144 weeks)

\begin{tabular}{l|ll}
\hline Study & IMMEDIATE & DEFERRED \\
\hline Ananworanich 2008 & -1.1 (IQR: -1.5 to -0.8$)$ & -1.0 (IQR: -1.7 to 0.2$)$ \\
\hline PREDICT 2012 & -1.27 (IQR: -1.78 to $-.0 .39)$ & -1.40 (IQR: -1.99 to -0.89$)$ \\
\hline
\end{tabular}

Analysis I.13. Comparison I IMMEDIATE versus DEFERRED initiation of ART all ages (RCT), Outcome I3 Mean height gain per year in $\mathrm{cm}$.

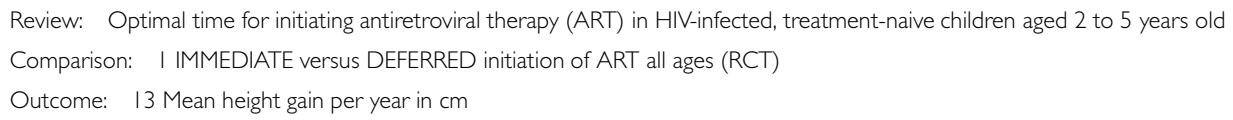

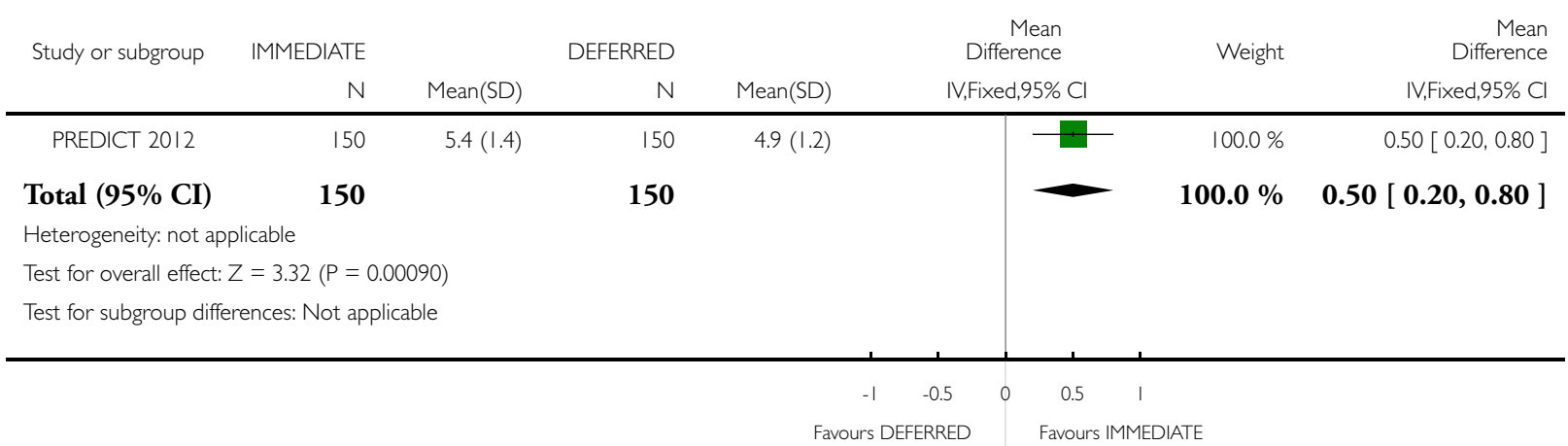

Analysis I.I4. Comparison I IMMEDIATE versus DEFERRED initiation of ART all ages (RCT), Outcome I4 Median height-for-age $Z$ score at study end (I 34 - I 44 weeks).

Median height-for-age $Z$ score at study end (134 - 144 weeks)

\begin{tabular}{lll}
\hline Study & IMMEDIATE & DEFERRED \\
\hline Ananworanich 2008 & -1.4 (IQR: -2.0 to -0.8$)$ & -0.8 (IQR: -1.3 to -0.4$)$ \\
\hline PREDICT 2012 & -1.50 (IQR: -2.35 to $-.0 .54)$ & -1.73 (IQR: -2.42 to -0.95$)$ \\
\hline
\end{tabular}

Optimal time for initiating antiretroviral therapy (ART) in HIV-infected, treatment-naive children aged 2 to 5 years old (Review) 
Analysis I.I5. Comparison I IMMEDIATE versus DEFERRED initiation of ART all ages (RCT), Outcome I5 Mean standardized score on Beery VMI at I44 weeks.

Review: Optimal time for initiating antiretroviral therapy (ART) in HIV-infected, treatment-naive children aged 2 to 5 years old

Comparison: I IMMEDIATE versus DEFERRED initiation of ART all ages (RCT)

Outcome: 15 Mean standardized score on Beery VMI at I 44 weeks

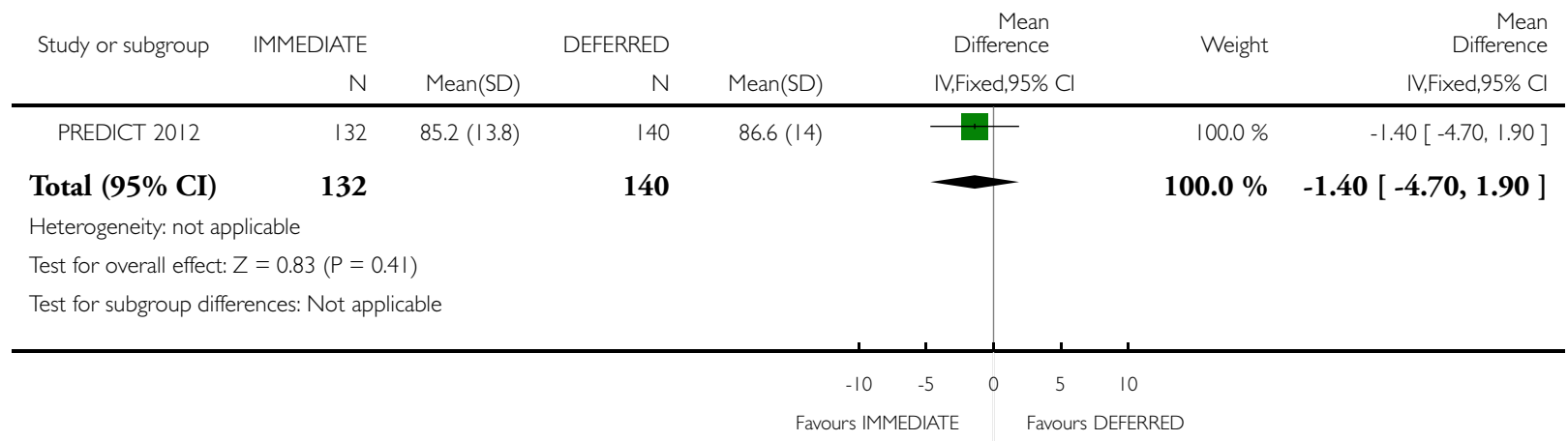

Analysis I.16. Comparison I IMMEDIATE versus DEFERRED initiation of ART all ages (RCT), Outcome I6 Proportion of children with adverse events.

Review: Optimal time for initiating antiretroviral therapy (ART) in HIV-infected, treatment-naive children aged 2 to 5 years old

Comparison: I IMMEDIATE versus DEFERRED initiation of ART all ages (RCT)

Outcome: 16 Proportion of children with adverse events

\begin{tabular}{|c|c|c|c|c|c|}
\hline Study or subgroup & IMMEDIATE & DEFERRED & $\begin{array}{r}\text { Risk Ratio } \\
\text { M- } \\
\mathrm{H} \text {,Random,95\% } \\
\mathrm{Cl}\end{array}$ & Weight & $\begin{array}{c}\text { Risk Ratio } \\
\text { M- } \\
\text { H,Random,95\% } \\
\text { Cl }\end{array}$ \\
\hline Ananworanich 2008 & $22 / 24$ & $19 / 19$ & & $100.0 \%$ & $0.92[0.80,1.07]$ \\
\hline Total $(95 \% \mathrm{CI})$ & 24 & 19 & $\bullet$ & $100.0 \%$ & $0.92[0.80,1.07]$ \\
\hline \multicolumn{6}{|c|}{ Total events: 22 (IMMEDIATE), I 9 (DEFERRED) } \\
\hline \multicolumn{6}{|c|}{ Heterogeneity: not applicable } \\
\hline \multicolumn{6}{|c|}{ Test for overall effect: $Z=1.06(P=0.29)$} \\
\hline Test for subgroup differe & ot applicable & & & & \\
\hline
\end{tabular}

$\begin{array}{ccccc}0.01 & 0.1 & 1 & 10 & 100 \\ \text { Favours IMMEDIATE } & & \text { Favours DEFERRED }\end{array}$

Optimal time for initiating antiretroviral therapy (ART) in HIV-infected, treatment-naive children aged 2 to 5 years old (Review) 
Analysis I.I7. Comparison I IMMEDIATE versus DEFERRED initiation of ART all ages (RCT), Outcome I7 Proportion of children with ART-related adverse events.

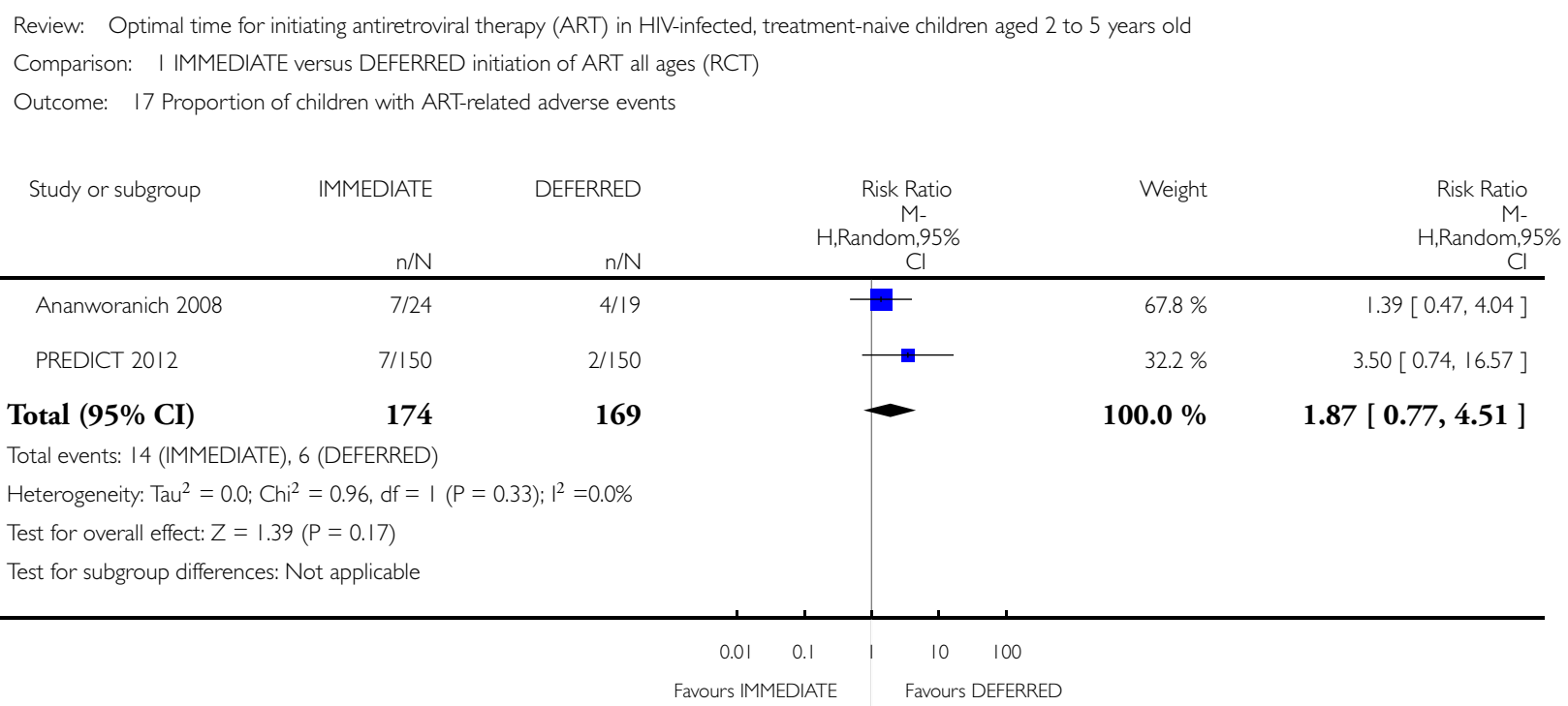

\section{Analysis 2.I. Comparison 2 SUBGROUP ANALYSIS: IMMEDIATE versus DEFERRED initiation of ART 24 to 59 months (RCT), Outcome I Death.}

Review: Optimal time for initiating antiretroviral therapy (ART) in HIV-infected, treatment-naive children aged 2 to 5 years old

Comparison: 2 SUBGROUP ANALYSIS: IMMEDIATE versus DEFERRED initiation of ART 24 to 59 months (RCT)

Outcome: I Death

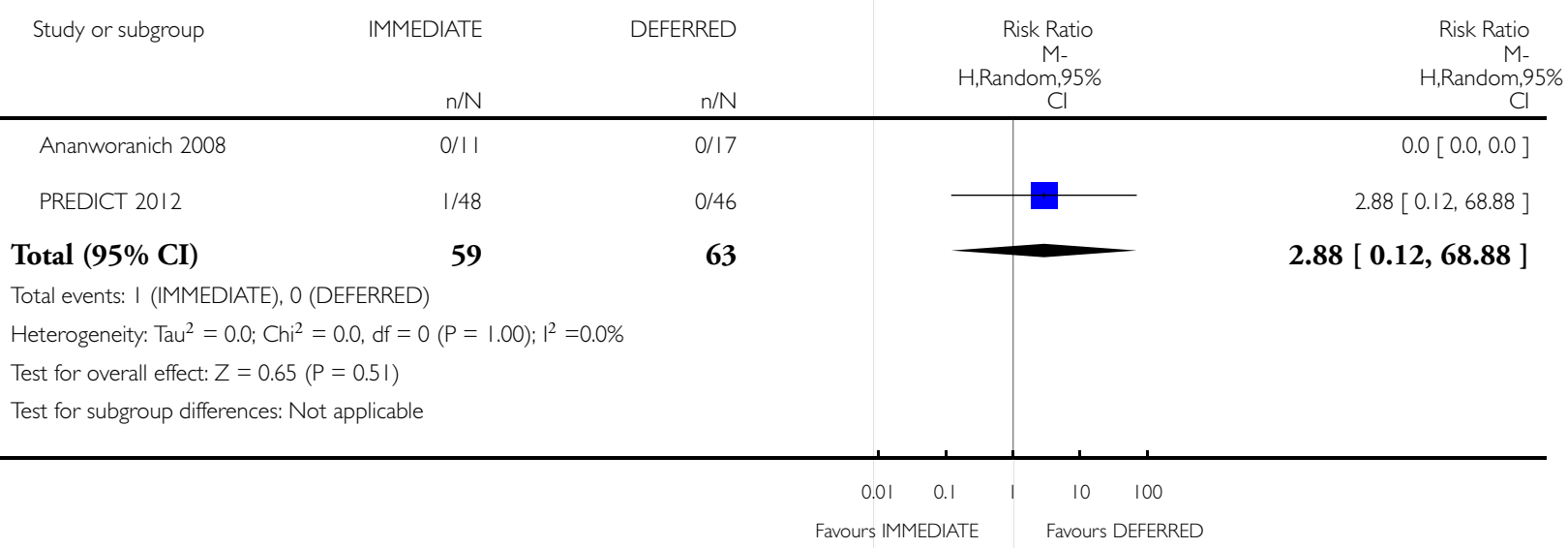

Optimal time for initiating antiretroviral therapy (ART) in HIV-infected, treatment-naive children aged 2 to 5 years old (Review) 
Analysis 2.2. Comparison 2 SUBGROUP ANALYSIS: IMMEDIATE versus DEFERRED initiation of ART 24 to 59 months (RCT), Outcome 2 CDC Category C disease (number of children).

Review: Optimal time for initiating antiretroviral therapy (ART) in HIV-infected, treatment-naive children aged 2 to 5 years old

Comparison: 2 SUBGROUP ANALYSIS: IMMEDIATE versus DEFERRED initiation of ART 24 to 59 months (RCT)

Outcome: 2 CDC Category C disease (number of children)

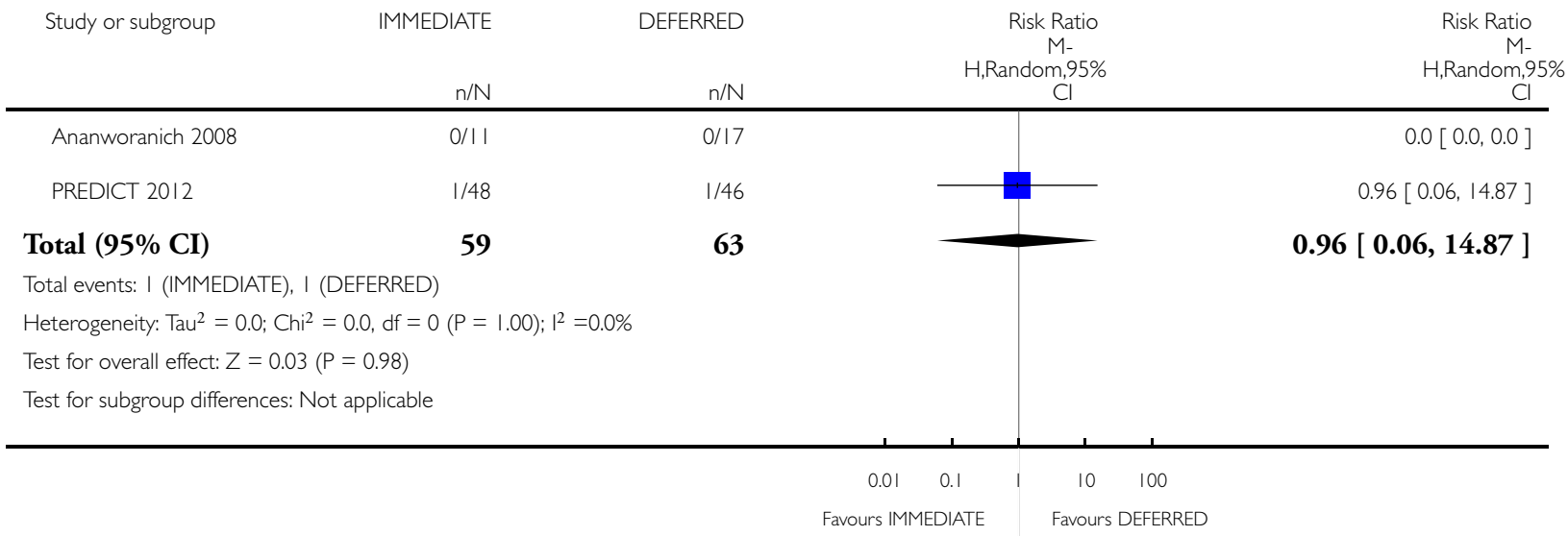

Analysis 2.3. Comparison 2 SUBGROUP ANALYSIS: IMMEDIATE versus DEFERRED initiation of ART 24 to 59 months (RCT), Outcome 3 CDC Category B disease (numbers of children) Relative Risk.

Review: Optimal time for initiating antiretroviral therapy (ART) in HIV-infected, treatment-naive children aged 2 to 5 years old

Comparison: 2 SUBGROUP ANALYSIS: IMMEDIATE versus DEFERRED initiation of ART 24 to 59 months (RCT)

Outcome: 3 CDC Category B disease (numbers of children) Relative Risk

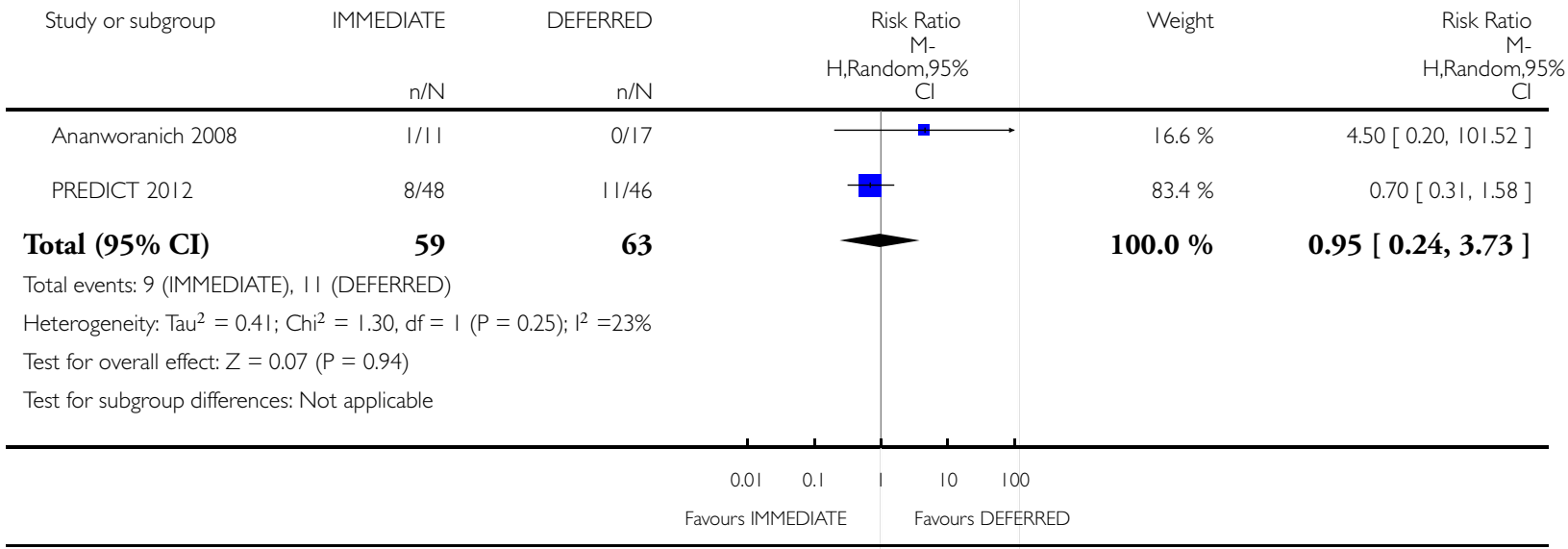

Optimal time for initiating antiretroviral therapy (ART) in HIV-infected, treatment-naive children aged 2 to 5 years old (Review) 
Analysis 2.4. Comparison 2 SUBGROUP ANALYSIS: IMMEDIATE versus DEFERRED initiation of ART 24 to 59 months (RCT), Outcome 4 CDC Category B disease (numbers of children) Peto Odds Ratio.

Review: Optimal time for initiating antiretroviral therapy (ART) in HIV-infected, treatment-naive children aged 2 to 5 years old

Comparison: 2 SUBGROUP ANALYSIS: IMMEDIATE versus DEFERRED initiation of ART 24 to 59 months (RCT)

Outcome: 4 CDC Category B disease (numbers of children) Peto Odds Ratio

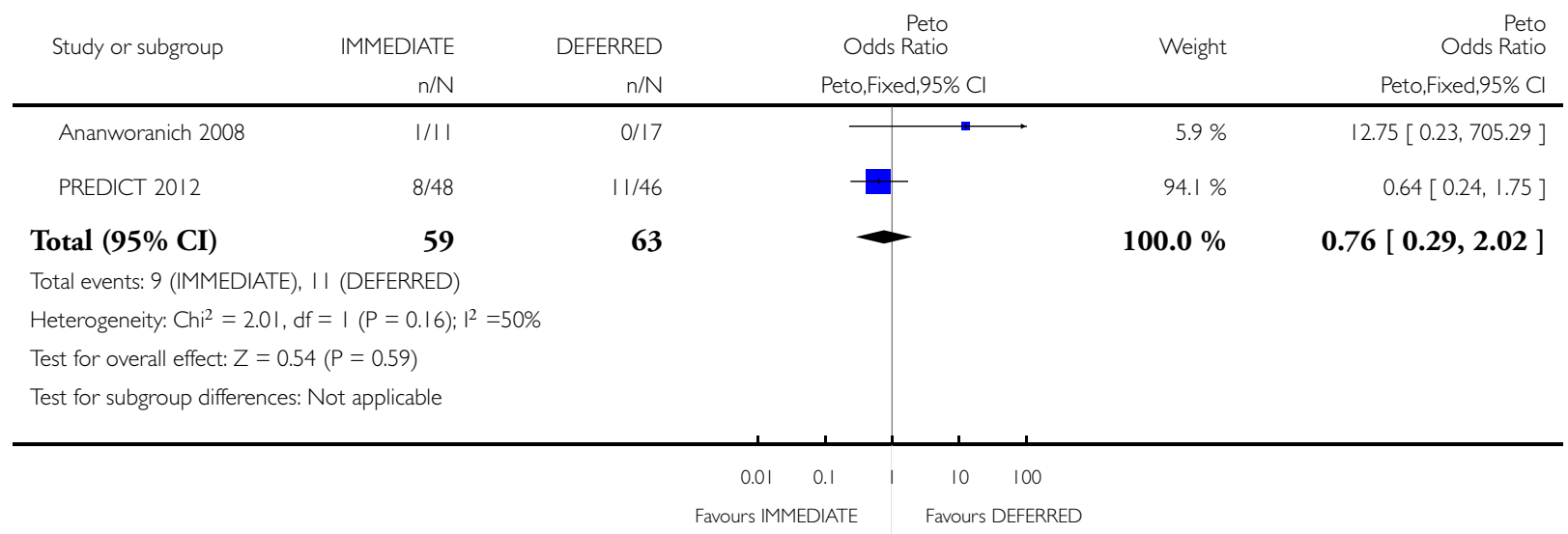




\section{Analysis 2.5. Comparison 2 SUBGROUP ANALYSIS: IMMEDIATE versus DEFERRED initiation of ART 24}

to 59 months (RCT), Outcome 5 Pulmonary TB (clinically diagnosed).

Review: Optimal time for initiating antiretroviral therapy (ART) in HIV-infected, treatment-naive children aged 2 to 5 years old

Comparison: 2 SUBGROUP ANALYSIS: IMMEDIATE versus DEFERRED initiation of ART 24 to 59 months (RCT)

Outcome: 5 Pulmonary TB (clinically diagnosed)

\begin{tabular}{|c|c|c|c|c|c|}
\hline \multirow[t]{2}{*}{ Study or subgroup } & IMMEDIATE & DEFERRED & \multirow{2}{*}{ M-H,Fixed,95\% Cl } & Weight & Risk Ratio \\
\hline & $\mathrm{n} / \mathrm{N}$ & $\mathrm{n} / \mathrm{N}$ & & & M-H,Fixed,95\% Cl \\
\hline Ananworanich 2008 & $|/| \mid$ & $0 / 17$ & $\longrightarrow$ & $20.7 \%$ & $4.50[0.20,101.52]$ \\
\hline PREDICT 2012 & $0 / 48$ & $1 / 46$ & & $79.3 \%$ & $0.32[0.01,7.65]$ \\
\hline Total (95\% CI) & 59 & 63 & & $100.0 \%$ & $1.19[0.19,7.27]$ \\
\hline \multicolumn{6}{|c|}{ Total events: I (IMMEDIATE), I (DEFERRED) } \\
\hline \multicolumn{6}{|c|}{ Heterogeneity: $\mathrm{Chi}^{2}=1.36, \mathrm{df}=\mathrm{I}(\mathrm{P}=0.24) ; \mathrm{I}^{2}=26 \%$} \\
\hline \multicolumn{6}{|c|}{ Test for overall effect: $Z=0.18(P=0.85)$} \\
\hline Test for subgroup differe & ot applicable & & & & \\
\hline
\end{tabular}

Favours IMMEDIATE Favours DEFERRED

Analysis 2.6. Comparison 2 SUBGROUP ANALYSIS: IMMEDIATE versus DEFERRED initiation of ART 24 to 59 months (RCT), Outcome 6 Proportion of children on ART with HIV-RNA $<50$ copies $/ \mathrm{ml}$.

Review: Optimal time for initiating antiretroviral therapy (ART) in HIV-infected, treatment-naive children aged 2 to 5 years old

Comparison: 2 SUBGROUP ANALYSIS: IMMEDIATE versus DEFERRED initiation of ART 24 to 59 months (RCT)

Outcome: 6 Proportion of children on ART with HIV-RNA $<50$ copies $/ \mathrm{ml}$

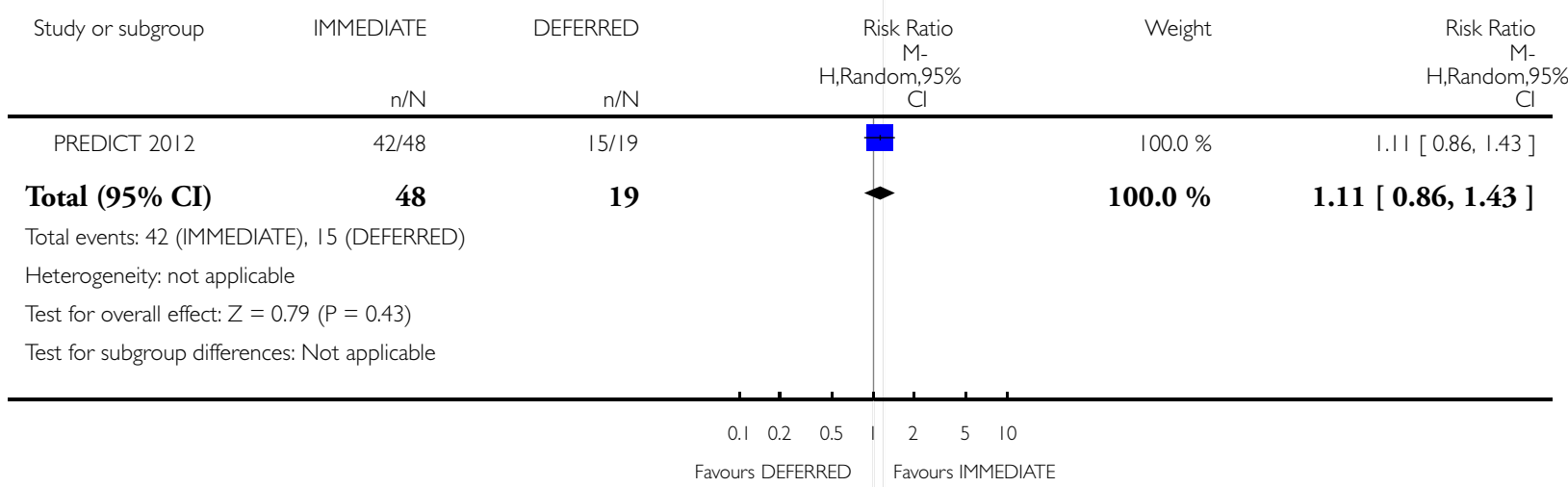


Analysis 2.7. Comparison 2 SUBGROUP ANALYSIS: IMMEDIATE versus DEFERRED initiation of ART 24 to 59 months (RCT), Outcome 7 Mean CD4\% at week I44.

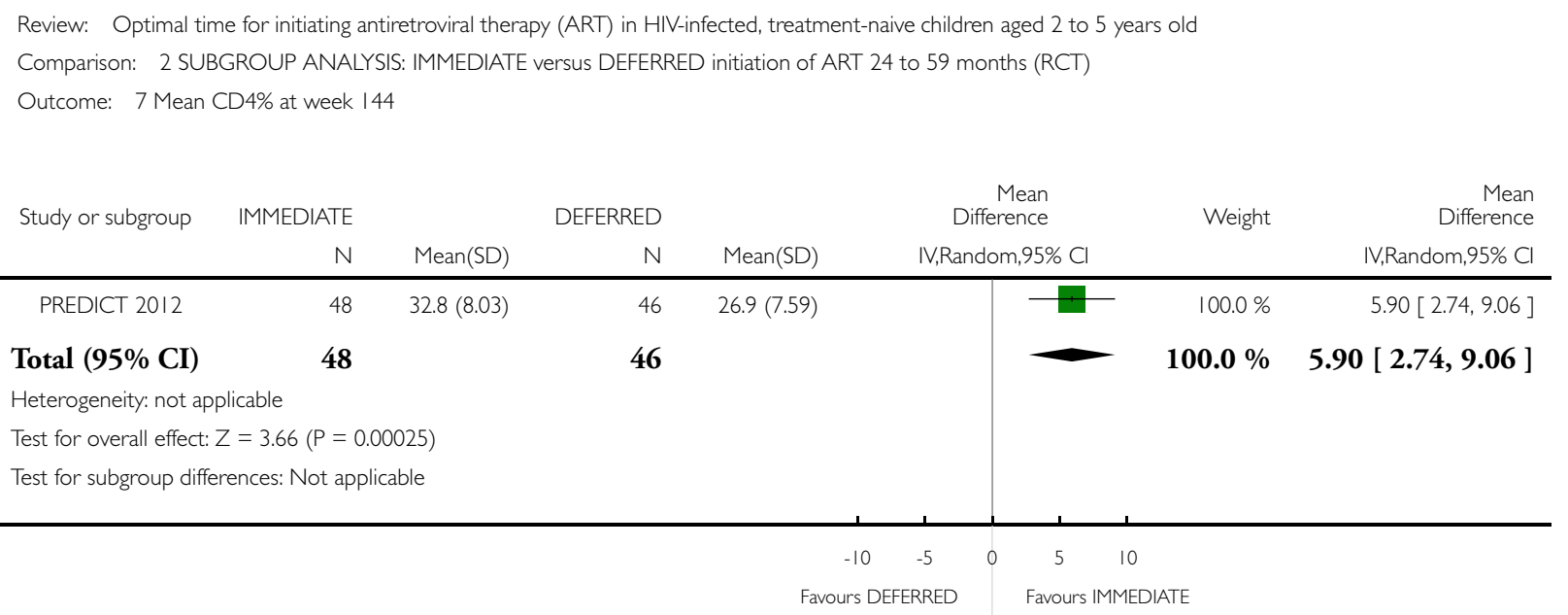

\section{Analysis 2.8. Comparison 2 SUBGROUP ANALYSIS: IMMEDIATE versus DEFERRED initiation of ART 24} to 59 months (RCT), Outcome 8 Proportion of children with CD4\% < I5\% at study end.

Review: Optimal time for initiating antiretroviral therapy (ART) in HIV-infected, treatment-naive children aged 2 to 5 years old

Comparison: 2 SUBGROUP ANALYSIS: IMMEDIATE versus DEFERRED initiation of ART 24 to 59 months (RCT)

Outcome: 8 Proportion of children with CD $4 \%<15 \%$ at study end

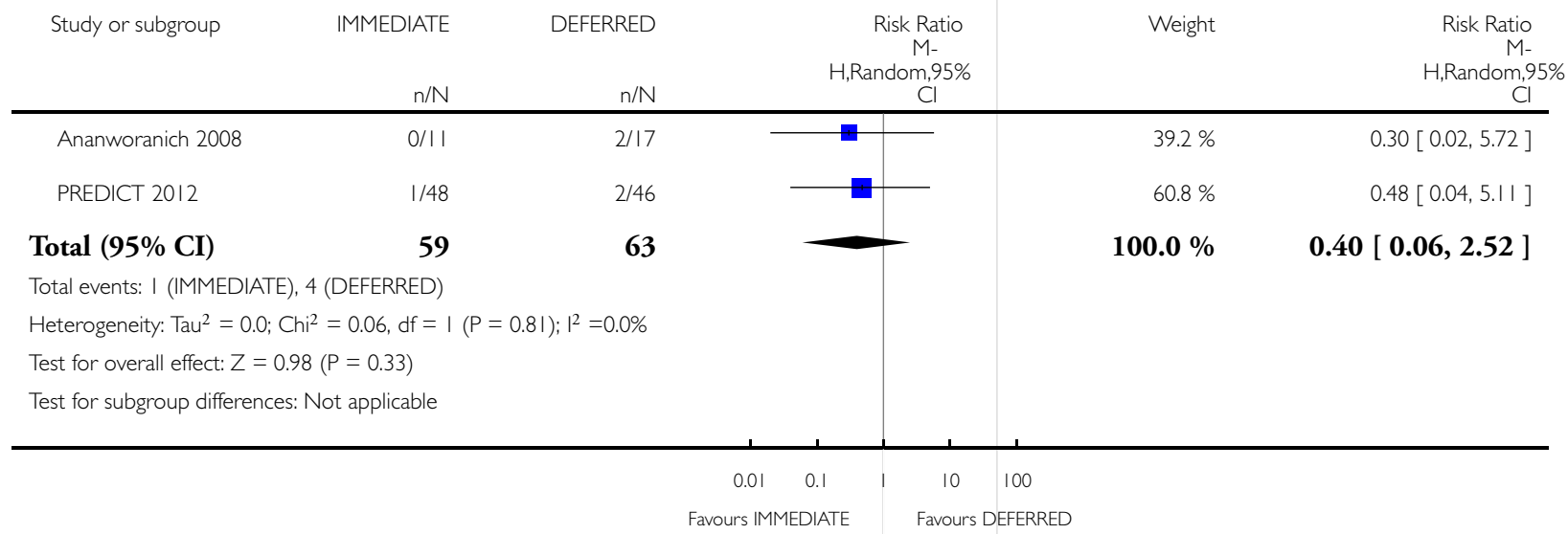

Optimal time for initiating antiretroviral therapy (ART) in HIV-infected, treatment-naive children aged 2 to 5 years old (Review) 
Analysis 2.9. Comparison 2 SUBGROUP ANALYSIS: IMMEDIATE versus DEFERRED initiation of ART 24 to 59 months (RCT), Outcome 9 Mean weight gain per year in kg.

Review: Optimal time for initiating antiretroviral therapy (ART) in HIV-infected, treatment-naive children aged 2 to 5 years old

Comparison: 2 SUBGROUP ANALYSIS: IMMEDIATE versus DEFERRED initiation of ART 24 to 59 months (RCT)

Outcome: 9 Mean weight gain per year in $\mathrm{kg}$

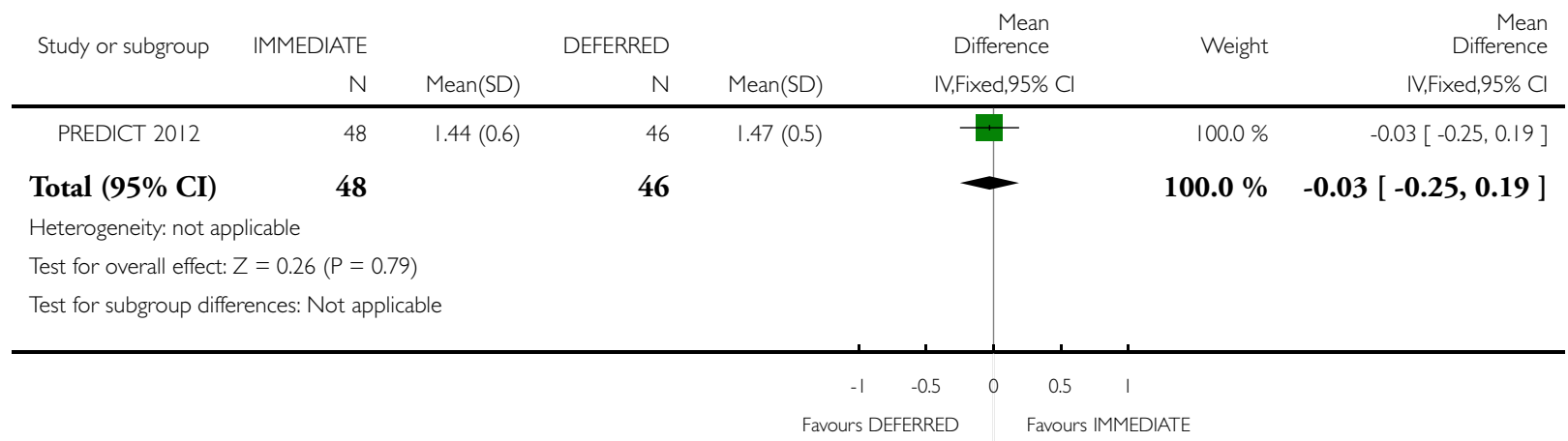

Analysis 2.10. Comparison 2 SUBGROUP ANALYSIS: IMMEDIATE versus DEFERRED initiation of ART 24 to 59 months (RCT), Outcome 10 Mean height gain per year in $\mathrm{cm}$.

Review: Optimal time for initiating antiretroviral therapy (ART) in HIV-infected, treatment-naive children aged 2 to 5 years old

Comparison: 2 SUBGROUP ANALYSIS: IMMEDIATE versus DEFERRED initiation of ART 24 to 59 months (RCT)

Outcome: 10 Mean height gain per year in $\mathrm{cm}$

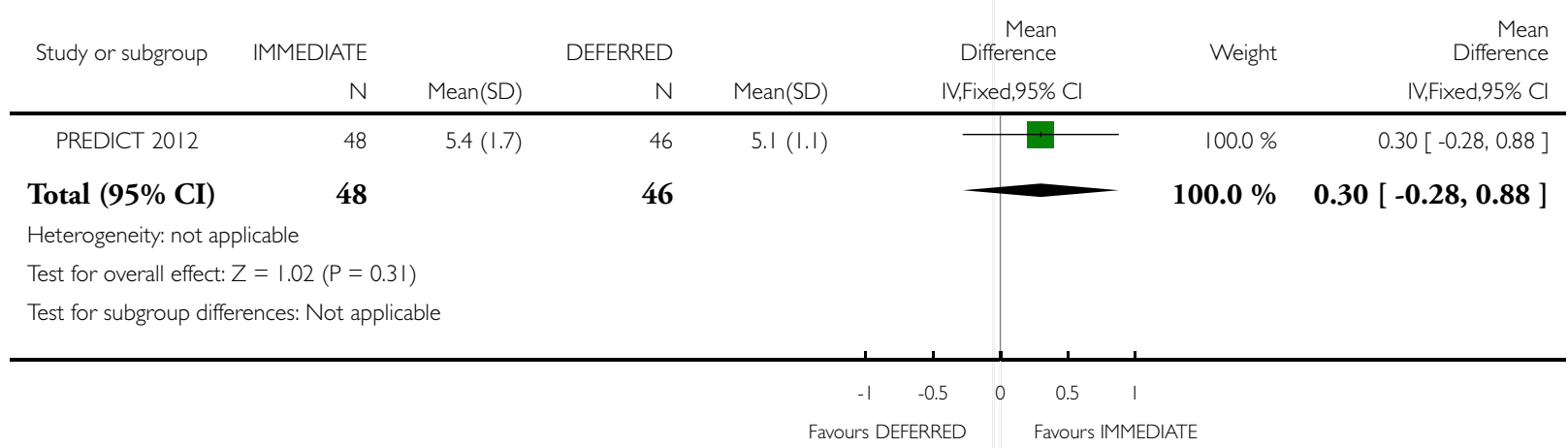

Optimal time for initiating antiretroviral therapy (ART) in HIV-infected, treatment-naive children aged 2 to 5 years old (Review) 
Analysis 2.11. Comparison 2 SUBGROUP ANALYSIS: IMMEDIATE versus DEFERRED initiation of ART 24 to 59 months (RCT), Outcome I I Mean standardized score on Beery VMI at I44 weeks.

Review: Optimal time for initiating antiretroviral therapy (ART) in HIV-infected, treatment-naive children aged 2 to 5 years old

Comparison: 2 SUBGROUP ANALYSIS: IMMEDIATE versus DEFERRED initiation of ART 24 to 59 months (RCT)

Outcome: II Mean standardized score on Beery VMI at I 44 weeks

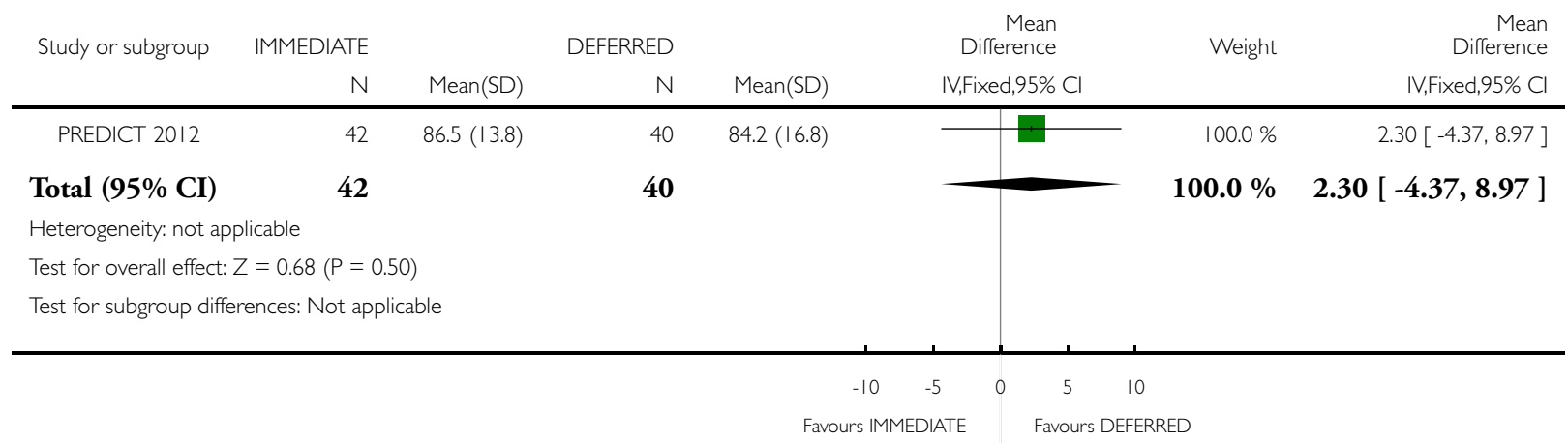

Analysis 2.12. Comparison 2 SUBGROUP ANALYSIS: IMMEDIATE versus DEFERRED initiation of ART 24 to 59 months (RCT), Outcome I 2 Proportion of children with ART-related Grade 3 or 4 adverse events.

Review: Optimal time for initiating antiretroviral therapy (ART) in HIV-infected, treatment-naive children aged 2 to 5 years old

Comparison: 2 SUBGROUP ANALYSIS: IMMEDIATE versus DEFERRED initiation of ART 24 to 59 months (RCT)

Outcome: 12 Proportion of children with ART-related Grade 3 or 4 adverse events

$\begin{array}{llll}\text { Study or subgroup } & \text { IMMEDIATE } & \text { REFERRED Ratio } & \text { Wisk Ratio }\end{array}$

\begin{tabular}{|c|c|c|c|c|c|}
\hline & $\mathrm{n} / \mathrm{N}$ & $\mathrm{n} / \mathrm{N}$ & $\begin{array}{c}\mathrm{H} \text {,Random,95\% } \\
\mathrm{Cl}\end{array}$ & & $\begin{array}{r}\mathrm{H}, \text { Random,959 } \\
\mathrm{Cl}\end{array}$ \\
\hline PREDICT 2012 & $1 / 48$ & $2 / 46$ & $+1+$ & $100.0 \%$ & $0.48[0.04,5.11]$ \\
\hline Total (95\% CI) & 48 & 46 & & $100.0 \%$ & $0.48[0.04,5.11]$ \\
\hline
\end{tabular}

Total events: I (IMMEDIATE), 2 (DEFERRED)

Heterogeneity: not applicable

Test for overall effect: $Z=0.61(P=0.54)$

Test for subgroup differences: Not applicable

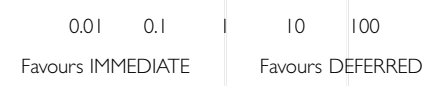

Optimal time for initiating antiretroviral therapy (ART) in HIV-infected, treatment-naive children aged 2 to 5 years old (Review) 


\section{Analysis 3.I. Comparison 3 ADJUSTED/WEIGHTED EARLY vs DEFERRED initiation of ART in children with TB and HIV, Outcome I Death.}

Review: Optimal time for initiating antiretroviral therapy (ART) in HIV-infected, treatment-naive children aged 2 to 5 years old

Comparison: 3 ADJUSTEDMEIGHTED EARLY vs DEFERRED initiation of ART in children with TB and HIV

Outcome: I Death

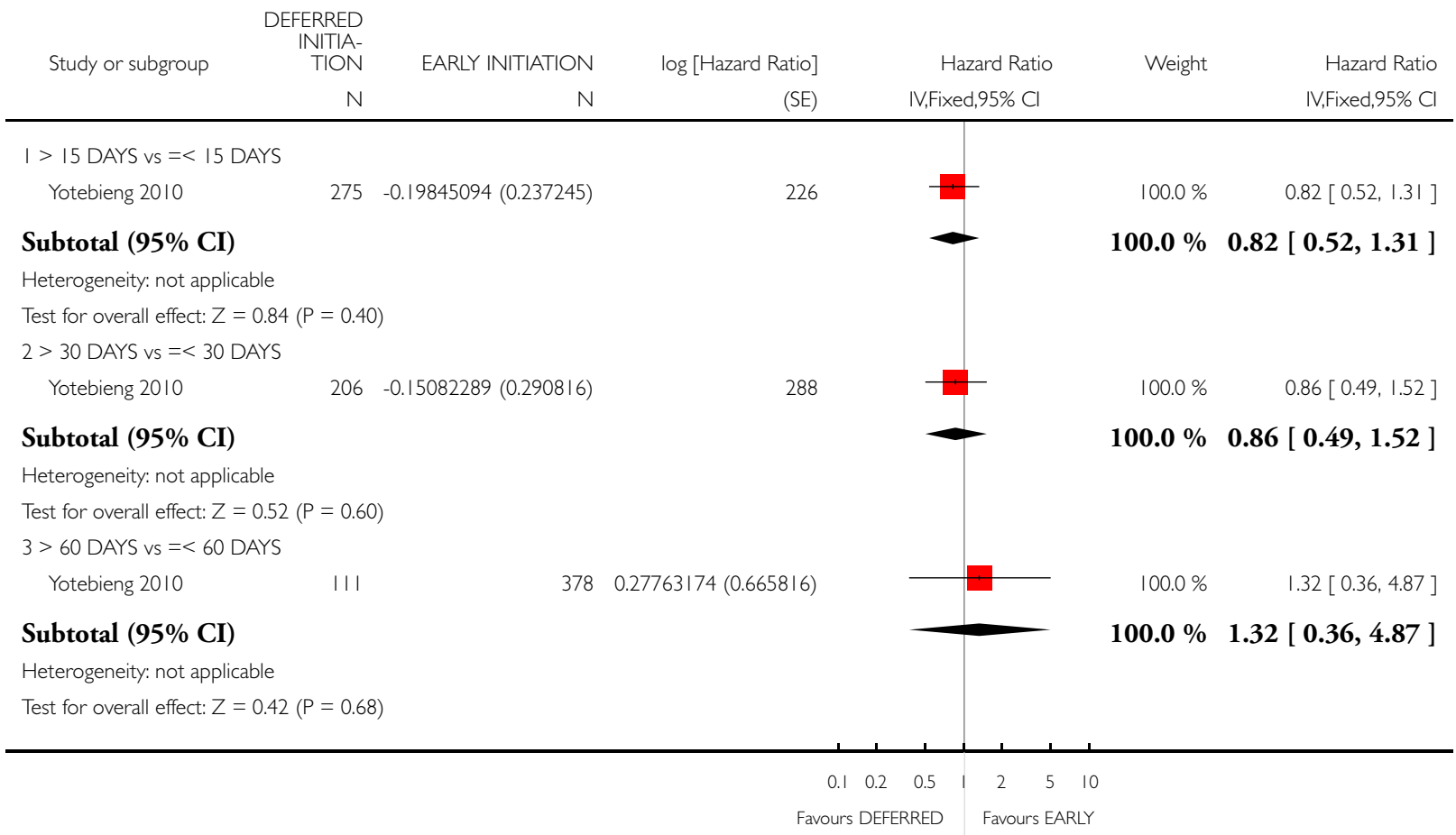


Analysis 3.2. Comparison 3 ADJUSTED/WEIGHTED EARLY vs DEFERRED initiation of ART in children with TB and HIV, Outcome 2 Virologic suppression.

Review: Optimal time for initiating antiretroviral therapy (ART) in HIV-infected, treatment-naive children aged 2 to 5 years old

Comparison: 3 ADJUSTEDMEIGHTED EARLY vs DEFERRED initiation of ART in children with TB and HIV

Outcome: 2 Virologic suppression

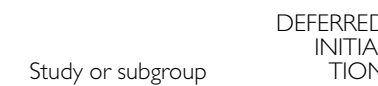

EARLY INITIATION log [Hazard Ratio]

Hazard Ratio

Weight

Hazard Ratio

$\mathrm{N}$

$\mathrm{N}$

IV,Fixed,95\% Cl

IV,Fixed,95\% Cl

I > I5 DAYS vs $=<15$ DAYS

Yotebieng 2010

I72-0.0202027| (0.12755 I)

152

$100.0 \%$

$0.98[0.76,1.26]$

Subtotal (95\% CI)

Heterogeneity: not applicable

Test for overall effect: $Z=0.16(P=0.87)$

$2>30$ DAYS vs $=<30$ DAYS

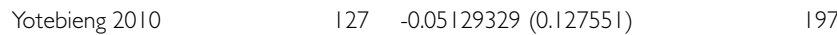

$107 \quad 100 \%$

$0.95[0.74,1.22]$

Subtotal (95\% CI)

Heterogeneity: not applicable

Test for overall effect: $Z=0.40(P=0.69)$

$3>60$ DAYS vs $=<60$ DAYS

Yotebieng 2010

$59-0.17435339(0.137755)$

265

$100.0 \%$

$0.84[0.64,1.10]$

Subtotal $(95 \% \mathrm{CI})$

Heterogeneity: not applicable

Test for overall effect: $Z=1.27(P=0.21)$

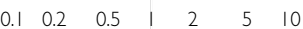

Favours EARLY Favours DEFERRED

A P PENDICES

Appendix I. Medline search strategy for RCT records

\begin{tabular}{ll|l}
\hline Search & Query & Items found \\
\hline$\# 9$ & $\begin{array}{l}\text { Search (\#6 AND \#7) AND (“1980/01/01”[Date - Publica- } \\
\text { tion] : “2012/06/27”[Date - Publication]) }\end{array}$ & 914 \\
\hline$\# 8$ & Search \#6 AND \#7 & 914
\end{tabular}

Optimal time for initiating antiretroviral therapy (ART) in HIV-infected, treatment-naive children aged 2 to 5 years old (Review)

Copyright (C) 2013 The Cochrane Collaboration. Published by John Wiley \& Sons, Ltd. 
\#7 Search infant[mh] OR infant*[tiab] OR toddler*[tiab] OR

2158897 child[mh] OR child*[tiab] OR preschool*[tiab] OR preschool*[tiab] OR schoolchild*[tiab] OR paediatric*[tiab] OR pediatric*[tiab]

\#6 Search \#3 AND \#4 AND \#5

\#5 Search optimal time[tiab] OR optimal timing[tiab] OR op1155983 timum time[tiab] OR optimum timing[tiab] OR time factors[mh] OR time factor[tiab] OR time factors[tiab] OR initiate[tiab] OR initiating[tiab] OR initiation[tiab] OR "when to start" [tiab] OR early treatment[tiab] OR deferred treatment[tiab] OR earlier treatment[tiab] OR best time[tiab] OR best timing[tiab] OR (start*[tiab] AND therapy[tiab])

\#4 Search (randomized controlled trial [pt] OR controlled clin-

2566592 ical trial [pt] OR randomized [tiab] OR placebo [tiab] OR drug therapy [sh] OR randomly [tiab] OR trial [tiab] OR groups [tiab]) NOT (animals [mh] NOT humans [mh])

\#2 Search antiretroviral therapy, highly active[MeSH] OR antiretroviral agents[MeSH] OR antiviral agents[MeSH:NoExp] OR ((anti[tiab]) AND (hiv[tiab])) OR antiretroviral*[tiab] OR ((anti[tiab]) AND (retroviral* ${ }^{*}$ tiab])) OR HAART[tiab] OR ((anti[tiab]) AND (acquired immunodeficiency[tiab])) OR ((anti[tiab]) AND (acquired immuno-deficiency[tiab])) OR ((anti[tiab]) AND (acquired immune-deficiency[tiab])) OR ((anti[tiab]) AND (acquired immun*[tiab]) AND (deficiency[tiab]))

\#1 Search HIV Infections[MeSH] OR HIV[MeSH] OR hiv[tiab] OR hiv-1*[tiab] OR hiv-2*[tiab] OR hiv1[tiab] OR hiv2[tiab] OR hiv infect*[tiab] OR human immunodeficiency virus[tiab] OR human immunedeficiency virus[tiab] OR human immuno-deficiency virus[tiab] OR human immune-deficiency virus[tiab] OR ((human immun*[tiab]) AND (deficiency virus[tiab])) OR acquired immunodeficiency syndrome[tiab] OR acquired immunedeficiency syndrome[tiab] OR acquired immuno-deficiency syndrome[tiab] OR acquired immune-deficiency syndrome[tiab] OR ((acquired immun*[tiab]) AND (deficiency syndrome[tiab])) OR "sexually transmitted diseases, Viral"[MeSH:NoExp] 
Appendix 2. EmBase search strategy for RCT records

\begin{tabular}{ll|l}
\hline No. & Query & Results \\
\hline$\# \mathbf{1 4}$ & \#11 AND \#12 AND [embase]/lim AND [1980-2012]/py & 616 \\
\hline$\# \mathbf{1 3}$ & $\# 11$ AND \#12 & 696 \\
\hline \#12 & $\begin{array}{l}\text { 'infant' OR 'infant'/de OR infant OR infant*:ab,ti OR 'tod- } \\
\text { dler' OR 'toddler'/de OR toddler OR toddler*:ab,ti OR 'child' } \\
\text { OR 'child'/de OR child OR child*:ab,ti OR 'preschool child'/ } \\
\text { de OR 'preschool child' OR preschool*:ab,ti OR pre+school*: } \\
\text { ab,ti OR 'school child'/de OR 'school child' OR schoolchild*: } \\
\text { ab,ti OR paediatric:ab,ti OR pediatric*:ab,ti }\end{array}$ & \\
\hline \#11 & \begin{tabular}{l} 
\#3 AND \#9 AND \#10 \\
\hline
\end{tabular} & 4287 \\
\hline
\end{tabular}

\#10 'optimal time':ab,ti OR 'optimal timing'ab,ti OR 'optimum 2769213 time':ab,ti OR 'optimum timing':ab,ti OR 'time'/de OR time OR 'time factor':ab,ti OR 'time factors':ab,ti OR initiate:ab,ti OR initiating:ab,ti OR initiation:ab,ti OR 'when to start':ab, ti OR 'early treatment':ab,ti OR 'deferred treatment':ab,ti OR 'earlier treatment':ab,ti OR 'best time':ab,ti OR 'best timing': ab,ti OR (start* NEXT/3 therapy):ab,ti

\begin{tabular}{|c|c|c|}
\hline \#9 & \#4 NOT \#8 & 1579892 \\
\hline$\# 8$ & \#5 NOT \#7 & 1971197 \\
\hline \#7 & \#5 AND \#6 & 16771510 \\
\hline \#6 & 'human'/de OR 'human' & 17150693 \\
\hline \#5 & $\begin{array}{l}\text { 'animal'/de OR 'animal' OR 'nonhuman'/de OR 'nonhuman' } \\
\text { OR 'animal experiment'/de OR 'animal experiment' }\end{array}$ & 18742707 \\
\hline$\# 4$ & $\begin{array}{l}\text { 'randomized controlled trial'/de OR 'randomized controlled } \\
\text { trial' OR random*:ab,ti OR trial:ti OR allocat*:ab,ti OR fac- } \\
\text { torial*:ab,ti OR placebo*:ab,ti OR assign*:ab,ti OR volun- } \\
\text { teer*:ab,ti OR 'crossover procedure'/de OR 'crossover proce- } \\
\text { dure' OR 'double-blind procedure'/de OR 'double-blind pro- } \\
\text { cedure' OR 'single-blind procedure'/de OR 'single-blind pro- } \\
\text { cedure' OR (doubl* NEAR/3 blind*):ab,ti OR (singl*:ab,ti } \\
\text { AND blind*:ab,ti) OR crossover*:ab,ti OR cross+over*:ab,ti } \\
\text { OR (cross NEXT/1 over*):ab,ti }\end{array}$ & 1654771 \\
\hline \#3 & \#1 AND \#2 & 126378 \\
\hline
\end{tabular}


\#2 'human immunodeficiency virus vaccine'/de OR 'human immunodeficiency virus vaccine' OR 'human immunodeficiency virus vaccine':ab,ti OR 'anti human immunedeficiency':ab, ti OR 'anti human immunodeficiency':ab,ti OR 'anti human immuno-deficiency':ab,ti OR 'anti human immune-deficiency':ab,ti OR 'anti acquired immune-deficiency':ab,ti OR 'anti acquired immunedeficiency':ab,ti OR 'anti acquired immunodeficiency':ab,ti OR 'anti acquired immuno-deficiency': ab,ti OR 'anti hiv':ab,ti OR antiretrovir*:ab,ti OR 'anti retroviral':ab,ti OR 'anti retrovirals':ab,ti OR 'anti retrovirus':ab, ti OR haart:ab,ti OR 'aids vaccine':ab,ti OR 'aids vaccines': ab,ti OR 'anti human immunodeficiency virus agent'/de OR 'anti human immunodeficiency virus agent' OR 'antiretrovirus agent'/de OR 'antiretrovirus agent' OR 'highly active antiretroviral therapy'/de OR 'highly active antiretroviral therapy' OR 'highly active antiretroviral therapy':ab,ti

\#1 'human immunodeficiency virus infection'/exp OR 'human immunodeficiency virus infection'/de OR 'human immunodeficiency virus infection' OR 'human immunodeficiency virus'/exp OR 'human immunodeficiency virus'/de OR 'human immunodeficiency virus' $O R$ 'human immunodeficiency virus:ab,ti' OR hiv:ab,ti OR 'hiv-1':ab,ti OR 'hiv-2':ab,ti OR 'human immunodeficiency virus':ab,ti OR 'human immunedeficiency virus' :ab,ti OR 'human immune-deficiency virus': $a b$, ti OR 'human immuno-deficiency virus':ab,ti OR 'acquired immunodeficiency syndrome':ab,ti OR 'acquired immunodeficiency syndrome':ab,ti OR 'acquired immune-deficiency syndrome':ab,ti OR 'acquired immunedeficiency syndrome': ab,ti

\section{Appendix 3. CENTRAL search strategy for RCT records}

\begin{tabular}{lll}
\hline ID & Search & Hits \\
\hline$\# 1$ & MeSH descriptor HIV Infections explode all trees 6664 & MeSH descriptor HIV Infections explode all trees 6664 \\
\hline$\# 2$ & MeSH descriptor HIV explode all trees & 2235 \\
\hline$\# 3$ & $\begin{array}{l}\text { hiv OR hiv-1* OR hiv-2* OR hiv1 OR hiv2 OR HIV } \\
\text { INFECT* OR HUMAN IMMUNODEFICIENCY } \\
\text { VIRUS OR HUMAN IMMUNEDEFICIENCY VIRUS } \\
\text { OR HUMAN IMMUNE-DEFICIENCY VIRUS } \\
\begin{array}{l}\text { OR HUMAN IMMUNO-DEFICIENCY VIRUS } \\
\text { OR HUMAN IMMUN* DEFICIENCY VIRUS OR }\end{array}\end{array}$ & 10868 \\
\hline
\end{tabular}


ACQUIRED IMMUNODEFICIENCY SYNDROME

OR ACQUIRED IMMUNEDEFICIENCY SYNDROME

OR ACQUIRED IMMUNO-DEFICIENCY

SYNDROME OR ACQUIRED IMMUNE-

DEFICIENCY SYNDROME OR ACQUIRED IMMUN* DEFICIENCY SYNDROME

\begin{tabular}{|c|c|c|}
\hline \#4 & MeSH descriptor Lymphoma, AIDS-Related, this term only & 21 \\
\hline$\# 5$ & $\begin{array}{l}\text { MeSH descriptor Sexually Transmitted Diseases, Viral, this } \\
\text { term only }\end{array}$ & 22 \\
\hline \#6 & (\#1 OR \#2 OR \#3 OR \#4 OR \#5) & 10945 \\
\hline \#7 & $\begin{array}{l}\text { MeSH descriptor Antiretroviral Therapy, Highly Active, } \\
\text { this term only }\end{array}$ & 827 \\
\hline \#8 & MeSH descriptor Anti-HIV Agents explode all trees & 2222 \\
\hline$\# 9$ & MeSH descriptor Antiviral Agents, this term only & 2886 \\
\hline$\# 10$ & MeSH descriptor AIDS Vaccines, this term only & 284 \\
\hline \#11 & $\begin{array}{l}\text { ANTI HIV OR ANTIRETROVIRAL* OR ANTI } \\
\text { RETROVIRAL* OR AIDS VACCIN* }\end{array}$ & 4961 \\
\hline \#12 & (\#7 OR \#8 OR \#9 OR \#10 OR \#11) & 7832 \\
\hline \#13 & (\#6 AND \#12) & 5338 \\
\hline \#14 & MeSH descriptor Time Factors, this term only & 44585 \\
\hline \#15 & $\begin{array}{l}\text { time factor:ti,ab OR time factors:ti,ab OR optimal } \\
\text { time:ti,ab OR optimal timing:ti,ab OR initiate:ti,ab OR } \\
\text { initiating:ti,ab OR initiation:ti,ab OR “when to start”:ti,ab } \\
\text { OR early treatment:ti,ab OR deferred treatment:ti,ab OR } \\
\text { earlier treatment:ti,ab OR best time:ti,ab OR best } \\
\text { timing:ti,ab OR (start* NEXT/3 therapy):ti,ab }\end{array}$ & 51057 \\
\hline \#16 & (\#14 OR \#15) & 88247 \\
\hline \#17 & $\begin{array}{l}\text { infant:ti,ab,kw OR toddler:ti,ab,kw OR child:ti,ab,kw OR } \\
\text { preschool*:ti,ab,kw OR pre school:ti,ab,kw OR } \\
\text { schoolchild*:ti,ab,kw OR paediatric*:ti,ab,kw OR } \\
\text { pediatric*:ti,ab,kw }\end{array}$ & 77426 \\
\hline \#18 & (\#13 AND \#16 AND \#17) & 238 \\
\hline
\end{tabular}


Appendix 4. Medline search strategy for cohort records (excluding RCT records)

\begin{tabular}{|c|c|c|}
\hline Search & Query & Items found \\
\hline \#13 & $\begin{array}{l}\text { Search (\#10 NOT \#11) AND (“1980/01/01”[Date - Publi- } \\
\text { cation] : "2012/09/11”[Date - Publication]) }\end{array}$ & 130 \\
\hline \#12 & Search \#10 NOT \#11 & 130 \\
\hline \#11 & $\begin{array}{l}\text { Search (randomized controlled trial [pt] OR controlled clin- } \\
\text { ical trial [pt] OR randomized [tiab] OR placebo [tiab] OR } \\
\text { drug therapy [sh] OR randomly [tiab] OR trial [tiab] OR } \\
\text { groups [tiab]) NOT (animals [mh] NOT humans [mh]) }\end{array}$ & 2600091 \\
\hline \#10 & Search \#6 AND \#9 & 584 \\
\hline \#9 & Search \#7 OR \#8 & 1482738 \\
\hline$\# 8$ & $\begin{array}{l}\text { Search (follow up [tiab] OR prospective[tiab] OR longitudi- } \\
\text { nal[tiab]) AND (study[tiab] OR studies[tiab]) }\end{array}$ & 577814 \\
\hline \#7 & Search cohort studies[mh] OR cohort[tiab] & 1263329 \\
\hline \#6 & Search \#3 AND \#4 AND \#5 & 1271 \\
\hline \#5 & 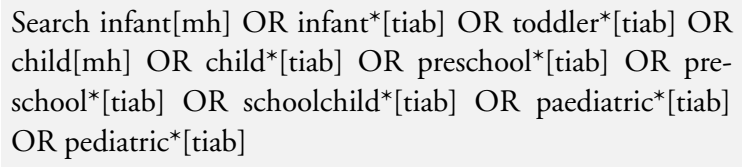 & 2177077 \\
\hline \#4 & $\begin{array}{l}\text { Search optimal time[tiab] OR optimal timing[tiab] OR op- } \\
\text { timum time[tiab] OR optimum timing[tiab] OR time fac- } \\
\text { tors[mh] OR time factor[tiab] OR time factors[tiab] OR ini- } \\
\text { tiate[tiab] OR initiating[tiab] OR initiation[tiab] OR "when } \\
\text { to start"[tiab] OR early treatment[tiab] OR deferred treat- } \\
\text { ment[tiab] OR earlier treatment[tiab] OR best time[tiab] OR } \\
\text { best timing[tiab] OR (start*[tiab] AND therapy[tiab]) }\end{array}$ & 1167527 \\
\hline \#3 & Search \#1 AND \#2 & 77367 \\
\hline \#2 & $\begin{array}{l}\text { Search antiretroviral therapy, highly active[MeSH] OR anti- } \\
\text { retroviral agents[MeSH] OR antiviral agents[MeSH:NoExp] } \\
\text { OR ((anti[tiab]) AND (hiv[tiab])) OR antiretroviral*[tiab] }\end{array}$ & 122889 \\
\hline
\end{tabular}


OR ((anti[tiab]) AND (retroviral*[tiab])) OR HAART[tiab] OR ((anti[tiab]) AND (acquired immunodeficiency[tiab])) OR ((anti[tiab]) AND (acquired immuno-deficiency[tiab])) OR ((anti[tiab]) AND (acquired immune-deficiency[tiab])) OR ((anti[tiab]) AND (acquired immun*[tiab]) AND (deficiency[tiab])) hiv[tiab] OR hiv-1*[tiab] OR hiv-2*[tiab] OR hiv1[tiab] OR hiv2[tiab] OR hiv infect*[tiab] OR human immunodeficiency virus[tiab] OR human immunedeficiency virus[tiab] OR human immuno-deficiency virus[tiab] OR human immune-deficiency virus[tiab] OR ((human immun*[tiab]) AND (deficiency virus[tiab])) OR acquired immunodeficiency syndrome[tiab] OR acquired immunedeficiency syndrome[tiab] OR acquired immuno-deficiency syndrome[tiab] OR acquired immune-deficiency syndrome[tiab] OR ((acquired immun*[tiab]) AND (deficiency syndrome[tiab])) OR "sexually transmitted diseases, Viral"[MeSH:NoExp]

\section{Appendix 5. EmBase search strategy for cohort records (excluding RCT records)}

\begin{tabular}{l|l|l}
\hline No. & Query & Results \\
\hline$\# \mathbf{1 5}$ & $\begin{array}{l}\text { \#8 NOT \#14 AND [embase]/lim AND }[1-1-1980] / \text { sd NOT } \\
{[11-9-2012] / \text { sd }}\end{array}$ & 950 \\
\hline$\# \mathbf{1 4}$ & $\# 9$ NOT \#13 & 1612308 \\
\hline \#13 & $\# 10$ NOT \#12 & 2100850 \\
\hline$\# \mathbf{1 2}$ & \#10 AND \#11 & 17006261 \\
\hline \#11 & 'human'/de OR 'human' & 17384855 \\
\hline \#10 & $\begin{array}{l}\text { 'animal'/de OR 'animal' OR 'nonhuman'/de OR 'nonhuman' } \\
\text { OR 'animal experiment'/de OR 'animal experiment' }\end{array}$ & 19107111 \\
\hline
\end{tabular}

\#9 'randomized controlled trial'/de OR 'randomized controlled 1689960 trial' OR random*:ab,ti OR trial:ti OR allocat*:ab,ti OR factorial*:ab,ti OR placebo*:ab,ti OR assign*:ab,ti OR volunteer*:ab,ti OR 'crossover procedure'/de OR 'crossover procedure' OR 'double-blind procedure'/de OR 'double-blind procedure' OR 'single-blind procedure'/de OR 'single-blind procedure' OR (doubl* NEAR/3 blind*):ab,ti OR (singl*:ab,ti 
AND blind*:ab,ti) OR crossover*:ab,ti OR cross+over*:ab,ti

OR (cross NEXT/1 over*):ab,ti

\begin{tabular}{lll} 
\#8 & \#6 AND \#7 & 1316 \\
\hline \#7 & \#3 AND \#4 AND \#5 & 3218 \\
\hline
\end{tabular}

\#6 'cohort analysis'/exp OR 'cohort analysis' OR 'longitudinal study'/exp OR 'longitudinal study' OR 'prospective study'/ exp OR 'prospective study' OR 'follow up'/exp OR 'follow up' OR cohort*:ab,ti OR 'longitudinal studies':ab,ti OR'prospective studies':ab,ti

\#5 'infant'/de OR infant*:ab,ti OR 'toddler'/de OR toddler*:ab, 2459580 ti OR 'child'/de OR child*:ab,ti OR 'preschool child'/de OR 'preschool child' OR preschool*:ab,ti OR pre+school*:ab,ti OR 'school child'/de OR schoolchild*:ab,ti OR paediatric:ab, ti OR pediatric*:ab,ti

\#4 'optimal time':ab,ti OR 'optimal timing':ab,ti OR 'optimum 2823933 time':ab,ti OR 'optimum timing':ab,ti OR 'time'/de OR time OR 'time factor':ab,ti OR 'time factors' $a b, t i$ OR initiate:ab,ti OR initiating:ab,ti OR initiation:ab,ti OR 'when to start':ab, ti OR 'early treatment':ab,ti OR 'deferred treatment':ab,ti OR 'earlier treatment':ab,ti OR 'best time':ab,ti OR 'best timing': ab,ti OR (start* NEXT/3 therapy):ab,ti

\section{1}

(4) 


\section{CONTRIBUTIONSOFAUTHORS}

All authors conceived the aim of the review and developed the protocol. NS and ME conducted the search eligibility, data extraction, and risk of bias assessment. MD acted as arbiter. NS conducted the analysis and all authors provided interpretation of results. NS and ME drafted the final review and all authors provided comment.

\section{DECLARATIONSOF INTEREST}

NS has no conflict of interest. ME and MD are investigators in the collaborative IeDEA cohort studies which evaluate the effects of cART on outcomes in HIV-infected children. MP is a consultant for the WHO and LM is an employee of the WHO.

\section{SOURCES OF SUPPORT}

\section{Internal sources}

- Centre for Infectious Disease Epidemiology and Research, School of Public Health, University of Cape Town, South Africa. Salary and material support

- Institute of Social Medicine, Institute of Social and Preventive Medicine, Bern, Switzerland.

Salary and material support

\section{External sources}

- No sources of support supplied 